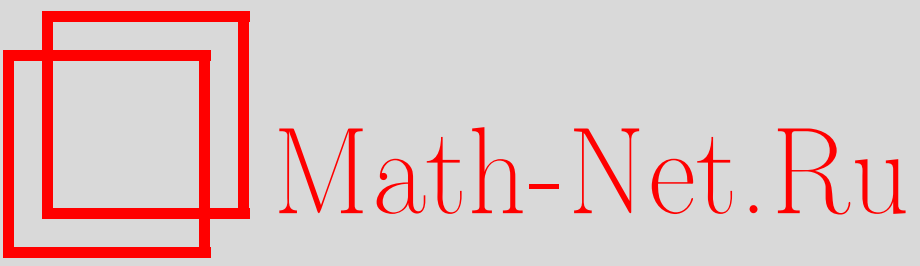

И. Ф. Красичков-Терновский, Спектральный синтез и аналитическое продолжение, УМН, 2003, том 58, выпуск 1, 33-112

DOI: https://doi.org/10.4213/rm593

Использование Общероссийского математического портала Math-Net.Ru подразумевает, что вы прочитали и согласны с пользовательским соглашением

http://www . mathnet.ru/rus/agreement

Параметры загрузки:

IP : 3.85 .183 .62

26 апреля 2023 г., 13:50:47

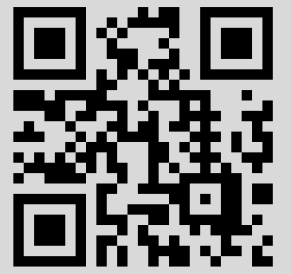




\title{
СПЕКТРАЛЬНЫЙ СИНТЕЗ И АНАЛИТИЧЕСКОЕ ПРОДОЛЖЕНИЕ
}

\author{
И. Ф. КРАСИЧКОВ-ТЕРНОВСКИЙ
}

\begin{abstract}
Рассматривается замкнутое подпространство функций, голоморфных в области $n$-мерного комплексного пространства. Предполагается, что подпространство инвариантно относительно операторов частного дифференцирования и что оно допускает спектральный синтез, т.е. совпадает с замыканием линейной оболочки совместных корневых элементов операторов частного дифференцирования, содержащихся в нем. Исследуются условия, при которых элементы инвариантного подпространства допускают аналитическое продолжение в область, более широкую, чем исходная. Геометрия этой области зависит как от исходной области, так и от наличия в аннуляторном подмодуле инвариантного подпространства функций, допускающих специальные оценки снизу. Подобная же задача рассматривается для топологических произведений инвариантных подпространств. Результаты применяются для аналитического продолжения решений однородных уравнений свертки.

Библиография: 59 названий.
\end{abstract}

\section{СОДЕРЖАНИЕ}

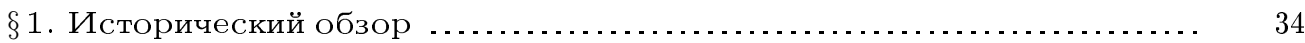

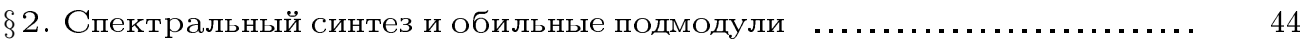

$\S 3$. Отображения инвариантных подпространств ................... 53

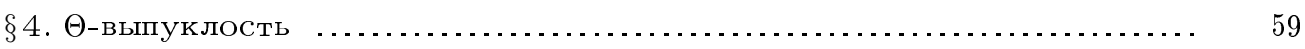

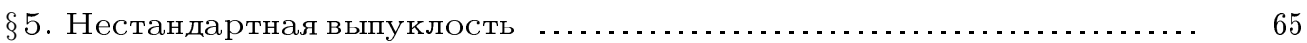

$\S 6$. Проекторы Дирихле ............................................ 73

$\S 7$. Мономорфизмы Дирихле ....................................... 81

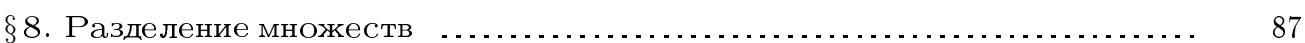

$\S 9$. Существование проекторов Дирихле в случае выпуклых $q$-областей 92

$\S 10$. Существование проекторов Дирихле в случае невыпуклых $q$-об-

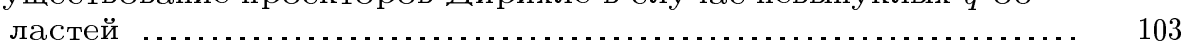

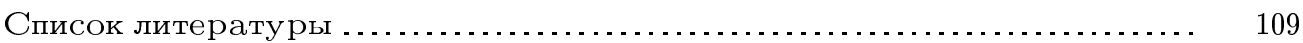

Работа выполнена при поддержке Российского фонда фундаментальных исследований (грант № 02-01-00030)

2 УМН, т. 58, вып. 1

(C) И. Ф. КРАСИЧКов-ТЕРНОВСКИЙ 2003 


\section{$\S 1$. Исторический обзор}

\section{1. Спектральный синтез.}

Пусть $\Omega=\left(\Omega_{1}, \ldots, \Omega_{q}\right)$ - система областей Рунге в $\mathbb{C}^{n}, \mathscr{H}_{1}=\mathscr{H}\left(\Omega_{1}\right), \ldots, \mathscr{H}_{q}=$ $\mathscr{H}\left(\Omega_{q}\right)$ - пространства функций, голоморфных в $\Omega_{1}, \ldots, \Omega_{q}$ соответственно, с топологиями равномерной сходимости на компактах, $\mathscr{H}=\mathscr{H}_{1} \times \cdots \times \mathscr{H}_{q}$ - топологическое произведение пространств $\mathscr{H}_{1}, \ldots, \mathscr{H}_{q}$. Элементами $\mathscr{H}$ являются векторы $f=$ $\left(f_{1}, \ldots, f_{q}\right)$, где $f_{1} \in \mathscr{H}_{1}, \ldots, f_{q} \in \mathscr{H}_{q}$. В пространстве $\mathscr{H}$ покомпонентно действуют операторы частного дифференцирования

$$
D_{1}=\frac{\partial}{\partial z_{1}}, \ldots, D_{n}=\frac{\partial}{\partial z_{n}} .
$$

Элемент $e \in \mathscr{H}$ называется корневым әлементом системы (1), отвечаюшим вектору собственных значений $\lambda=\left(\lambda_{1}, \ldots, \lambda_{n}\right)$, если е удовлетворяет системе уравнений вида

$$
\left(D_{1}-\lambda_{1} \mathrm{E}\right)^{m} e=0, \ldots,\left(D_{n}-\lambda_{n} \mathrm{E}\right)^{m} e=0,
$$

где $\mathrm{E}$ - тождественньй оператор, $m$ - целое положительное число. Корневые элементы, отвечаюшие вектору $\lambda=\left(\lambda_{1}, \ldots, \lambda_{n}\right)$, представляют собою так называемые әлементарные ехр-q-многочлены, т.е. элементы вида

$$
e=\left(e_{1}, \ldots, e_{n}\right),
$$

где $e_{i}(z)=p_{i}(z) \exp \langle\lambda, z\rangle, p_{i}(z)-$ многочлен переменных $z_{1}, \ldots, z_{n},\langle\lambda, z\rangle=\sum_{i=1}^{n} \lambda_{i} z_{i}$. Подпространство $W \subset \mathscr{H}$ назьвается инвариантныц, если оно замкнуто в $\mathscr{H}$ и удовлетворяет условию

$$
f \in W \Longrightarrow D_{1} f, \ldots, D_{q} f \in W .
$$

Инвариантное подпространство $W \subset \mathscr{H}$ допускает спектральный синтез, если $W$ совпадает с замьканием в топологии $\mathscr{H}$ линейной оболочки корневых элементов системы (1), содержащихся в $W$.

Инвариантные подпространства связаны с однородными уравнениями свертки. Пусть $\Omega$ - вьпуклая область в $\mathbb{C}^{n}$. Рассмотрим линейньй непрерьвньй функционал $S$ на $\mathscr{H}(\Omega)$. Известно, что функционал $S$ допускает интегральное представление

$$
\langle S, f\rangle=\int f d \mu,
$$

где $\mu$ - комплекснозначная мера, сосредоточенная на некотором компакте $K \Subset \Omega$. Из этого представления следует, что функционал $S$ можно применять не только к функциям $f \in \mathscr{H}(\Omega)$, но и к "малым" сдвигам этих функций, т.е. при "малых" $h=\left(h_{1}, \ldots, h_{n}\right)$ выражение $\langle S, f(z+h)\rangle$ имеет смысл. Это выражение представляет собою функцию $F(h)$, голоморфную в некоторой окрестности начала, скажем, $\omega$, и называется сверткой функционала $S$ и функции $f$, в обозначении $F=S * f$. Оператор свертки $f \rightarrow S * f$ линейно и непрерывно отображает $\mathscr{H}(\Omega)$ в $\mathscr{H}(\omega)$, кроме того, этот оператор коммутирует с операторами частного дифференширования $D_{1}, \ldots, D_{n}$.

Рассмотрим однородное уравнение свертки

$$
S * f=0 .
$$


Пусть $W$ обозначает совокупность решений $f \in \mathscr{H}(\Omega)$ этого уравнения. Из того, что оператор свертки $f \rightarrow S * f$ является линейньм и непрерьвным, следует, что $W$ есть замкнутое подпространство в $\mathscr{H}(\Omega)$; из того, что этот оператор коммутирует с операторами частного дифференцирования, следует, что $W$ - инвариантное подпространство. Другой пример - подпространство $W$ решений системы однородных уравнений свертки

$$
\left\{\begin{array}{c}
S_{1} * f=0 \\
\cdots \ldots \ldots \\
S_{m} * f=0
\end{array} .\right.
$$

Это подпространство инвариантно, так как оно представляет собою пересечение инвариантных подпространств $W_{1}, \ldots, W_{m}$ решений соответствуюших у равнений свертки $S_{1} * f=0, \ldots, S_{m} * f=0$. В случае $q>1$ примером инвариантного подпространства является совокупность решений $f=\left(f_{1}, \ldots, f_{q}\right) \in \mathscr{H}\left(\Omega_{1}\right) \times \cdots \times \mathscr{H}\left(\Omega_{q}\right)$ системы однородных сверточных уравнений с $q$ неизвестными функциями

$$
\left\{\begin{array}{c}
S_{1}^{(1)} * f_{1}+\cdots+S_{q}^{(1)} * f_{q}=0 \\
\cdots \ldots \ldots \ldots \ldots \ldots \ldots \ldots \ldots \ldots \\
S_{1}^{(m)} * f_{1}+\cdots+S_{q}^{(m)} * f_{q}=0
\end{array} .\right.
$$

3 десь $S_{j}^{(i)}$ - линейный непрерывный функционал на $\mathscr{H}\left(\Omega_{j}\right)$.

Задача спектрального синтеза - выяснить условия, при которых заданное инвариантное подпространство $W$ допускает спектральньй синтез.

Впервые задача спектрального синтеза была поставлена Л. Шварцем в 1947 г. в статье [1]. Там же Л. Шварц доказал, что каждое инвариантное подпространство в $\mathscr{H}(\mathbb{C})$ допускает спектральный синтез. Разные аспекты этой задачи в случае $n=1$ были исследованы автором в работах [2]-[9]. В случае $n>1$ известны лишь отдельные факты (см. [10]-[13]), в частности, инвариантное подпространство решений однородного уравнения свертки в выпуклой области допускает спектральный синтез [12], [13]. В работах А. Б. Шишкина [14] и автора [15] задача спектрального синтеза сводится к двойственной задаче локального описания.

Некоторые авторы $[16],[17]$ понимают термин “спектральный синтез" в более узком смысле, чем определено в настояшей работе. Спектральньй синтез для инвариантного подпространства $W \subset \mathscr{H}(\Omega)$, где $\Omega$ - область в $\mathbb{C}^{n}$, трактуется как совпадение $W$ с замьканием в топологии $\mathscr{H}(\Omega)$ линейной оболочки множества экспоненциальных одночленов, т.е. функций вида $z^{\alpha} \exp \langle\lambda, z\rangle$ содержашихся в $W$. Такая трактовка правомерна в случае $n=1$ и в случае инвариантного подпространства, связанного с одним уравнением свертки (см. [11]), так как в этих ситуациях из того, что инвариантное подпространство содержит ехр-многочлен, следует, что оно содержит ехр-одночлены, его составляюшие. При $n>1$ это, вообще говоря, уже не так. Класс инвариантных подпространств, допускаюших спектральный синтез в узком смысле, значительно сужается; из него исключаются некоторые простейшие инвариантные подпространства, связанные с системами однородных уравнений в частных производных. Вот соответствуюший пример, навеянный Л. Эренпрайсом [18]. Рассмотрим инвариантное под- 
пространство $W \subset \mathscr{H}\left(\mathbb{C}^{2}\right)$ решений $f$ системы трех уравнений в частных производHoIX

$$
\frac{\partial^{2}}{\partial z_{1}^{2}} f=0, \quad \frac{\partial^{2}}{\partial z_{2}^{2}} f=0, \quad \frac{\partial f}{\partial z_{1}}-\frac{\partial f}{\partial z_{2}}=0 .
$$

Методом разложения в ряд Тейлора легко установить, что общее решение этой системы имеет вид $f(z)=a+b\left(z_{1}+z_{2}\right)$, где $a, b$-произвольные константы. Совокупность функций такого вида представляет собою инвариантное подпространство, которое, очевидно, допускает спектральный синтез. Однако это подпространство не содержит одночлены $z_{1}, z_{2}$ и, значит, не допускает спектральньй синтез в узком смысле.

Ниже мы будем всегда предполагать, что $W$ - замкнутое инвариантное подпространство, допускающее спектральный синтез.

\section{2. Аналитическое продолжение.}

В настоящей статье рассматривается следующая задача: при каких условиях каждый элемент $f=\left(f_{1}, \ldots, f_{q}\right) \in W$ допускает аналитическое продолжение в более широкую, чем $\Omega$, систему областей $\Omega^{\prime}=\left(\Omega_{1}^{\prime}, \ldots, \Omega_{q}^{\prime}\right)$, т.е. каждая компонента $f_{i}$ продолжается в область $\Omega_{i}^{\prime} \supset \Omega_{i}, i=1, \ldots, q$ ?

Сама возможность аналитического продолжения проистекает из того, что при определенных условиях каждая последовательность линейных комбинаций элементарных $\operatorname{exp-q-многочленов~из~} W$, сходяшаяся к элементу $f \in W$ равномерно внутри $\Omega$, будет автоматически сходиться внутри более широкой системы областей $\Omega^{\prime}$; в результате элемент $f$ аналитически продолжается в $\Omega^{\prime}$. Это обстоятельство накладьвает определенные геометрические ограничения на области существования компонент $f_{1}, \ldots, f_{q}$ элемента $f$. Процесс аналитического продолжения сводится к расширению исходной системы областей $\Omega$ до тех пор, пока ее компоненты не приобретут требуемые геометрические свойства.

С каждьм инвариантньм подпространством $W \subset \mathscr{H}$ определенньм образом связывается его аннуляторный подмодуль $I$ - замкнутый подмодуль специального модуля $P$ целых функций экспоненциального типа (см. $\S 2$, п. 2). Геометрия системы областей $\Omega^{\prime}=\left(\Omega_{1}^{\prime}, \ldots, \Omega_{q}^{\prime}\right)$, куда осушествляется аналитическое продолжение, зависит от множества предельных направлений, по которым спектральное множество $(\S 2$, п. 3 ) инвариантного подпространства уходит в $\infty$, и от наличия в $I$ системы элементов, допускаюших определенную оценку снизу.

\section{3. История задачи аналитического продолжения при $n=1$.}

В случае $q=n=1$ задача имеет долгую историю. Ее истоки лежат в начатых еше в позапрошлом веке исследованиях областей сушествования функций, представимых в виде степенных рядов. В 1892 г. Ж. Адамар [19] доказал, что если функция $f$ представляется рядом

$$
f(z)=\sum a_{n} z^{\lambda_{n}}
$$

с пропусками $\lambda_{n+1}-\lambda_{n}>\theta \lambda_{n}$, где $\theta$ - положительное число, не зависящее от $n$, то гранища круга сходимости ряда (2) является естественной границей области существования функции $f$, т.е. каждая точка этой границы является особой для $f$. В терминах аналитического продолжения этот результат можно трактовать следующим образом: если функция $f$ голоморфна в области $G$, содержашей начало, и разлагается в 
ряд (2) с пропусками Адамара в некоторой окрестности начала, то $f$ допускает аналитическое продолжение в наименьший открытый круг с центром в начале, содержаший область $G$.

Е. Фабри [20] в 1896 г. доказал, что утверждение Адамара сохраняется при более общем условии на последовательность показателей, а именно, последовательность $\left\{\lambda_{n}\right\}$ должна иметь нулевую плотность:

$$
\lim _{n \rightarrow \infty} \frac{n}{\lambda_{n}}=0 .
$$

В работах Д. Пойа [21], А. Островского [22], В. Бернштейна [23] этот результат был распространен на ряды Дирихле: если функция $f$ представляется в виде ряда Дирихле

$$
f(z)=\sum a_{n} e^{-\lambda_{n} z}
$$

с последовательностью положительных показателей, имеющей нулевую плотность, то вертикальная прямая, ограничиваюшая наибольшую полуплоскость голоморфности функции $f$, является естественной гранищей области существования этой функции. Последний результат является частным случаем более общего утверждения Д. Пойа [21]: область сушествования решения однородного уравнения свертки $S * f=0$ с характеристической функцией $\varphi(\lambda)=\langle S, \exp \lambda z\rangle$ минимального типа является вьпуклой. Таким образом, если известно a priori, что решение такого уравнения голоморфно в некоторой области, то оно допускает аналитическое продолжение в вьпуклую оболочку этой области. В. Бернштейн $[23 ;$ гл. VI] установил, что если последовательность положительных показателей $\left\{\lambda_{n}\right\}$ имеет конечную максимальную плотность $\sigma$, то каждый замкнутый отрезок длины $2 \pi \sigma$ на прямой голоморфности ряда (3) содержит особую точку его суммы $f$. Отсюда, в частности, следует обобщение теоремы Адамара-Фабри: если последовательность $\left\{\lambda_{n}\right\}$ степеней ряда (2) имеет плотность $\sigma=\lim n / \lambda_{n}$, то каждая замкнутая дуга границы круга сходимости углового раствора $2 \pi \sigma$ содержит особую точку его суммы $f$. Отметим, что утверждение В. Бернштейна несколько ранее было доказано Д. Пойа [24] при условии разделения показателей:

$$
\left|\lambda_{n+1}-\lambda_{n}\right| \geqslant q>0 .
$$

А. Ф. Леонтьев [25], [26], развивая цитированные вьше результаты В. Бернштейна, доказал, что если характеристическая функция однородного уравнения свертки $S * f=0$ имеет вид

$$
\varphi(\lambda)=\prod\left(1-\left(\frac{z}{\lambda_{n}}\right)^{2}\right),
$$

где $n / \lambda_{n} \rightarrow \sigma>0$, то всякое решение $f$ этого уравнения, голоморфное в окрестности вертикального отрезка $[-i \pi \sigma, i \pi \sigma]$, допускает аналитическое продолжение в вертикальную полосу, содержашую этот отрезок; при этом вертикальные прямые, ограничивающие полосу голоморфности $f$, обладают свойством: всякий вертикальный отрезок длины $2 \pi \sigma$, лежаший на этих прямых, содержит особые точки $f$. Позднее такой же результат был получен Ж.-Р. Каханом [27]. Результату А. Ф. Леонтьева можно придать следуюшую геометрическую интерпретацию: если решение $f$ уравнения 
$S * f=0$ с характеристической функцией (4) голоморфнн в области $G$, содержащей отрезок $I=[-i \pi \sigma, i \pi \sigma]$, то $f$ допускает аналитическое продолжение в полосу

$$
G^{\prime}=(G \ominus I)_{0}+i \mathbb{R}
$$

где $G \ominus I$ - геометрическая разность $G$ и $I,(G \ominus I)_{0}$ - компонента связности этого множества, содержашая начало.

В цитированных вьше результатах аналитическое продолжение сопровождается распространением сходимости последовательностей полиномов Дирихле, причем в ту же область, в которую имеет место аналитическое продолжение. Например, в ситуации, рассмотренной А. Ф. Леонтьевым, если последовательность полиномов Дирихле сходится в области $G$, содержащей отрезок $[-i \pi \sigma, i \pi \sigma]$, то она сходится и в области (5). Задача распространения сходимости имеет самостоятельньй интерес, выходяший за рамки аналитического продолжения. В разных ситуациях эта задача рассматривалась А. Ф. Леонтьевым [28]-[30], Л. Шварцем [31], Ж.-Р. Каханом [27], А. Байетт [32], [33], И. Ф. Красичковым [34]. Отметим следуюший результат из работы [34].

Пусть система экспонент $\left\{e^{-\lambda_{n} z}\right\}, \lambda_{n}>0$, не полна в некоторой односвязной области $G$, т.е. замькание линейной оболочки этой системы в топологии $\mathscr{H}(G)$ не совпадает со всем $\mathscr{H}(G)$. Тогда для того чтобы сходимость последовательности полиномов Дирихле, т.е. конечных линейных комбинаций вида

$$
\sum a_{n} e^{-\lambda_{n} x}
$$

распространялась в некоторую полуплоскость $\operatorname{Re} z>a$, необходимо и достаточно, чтобы последовательность $\Lambda=\left\{\lambda_{n}\right\}$ имела конечньй индекс концентрации

$$
I=\lim _{\varepsilon \rightarrow 0} \varlimsup_{x \rightarrow \infty} \frac{1}{x} \int_{\varepsilon}^{1} \frac{n_{\sigma}(x)}{\sigma} d \sigma,
$$

где $n_{\sigma}(x)$ - число точек $\lambda_{n}$ в круге $e_{\sigma}(x)=\{z:|z-x| \leqslant \sigma|x|\}$.

В работе автора [35] было установлено, что геометрия области $G$, в которую продолжаются функции из инвариантного подпространства $W \subset \mathscr{H}(G)$, определяется множеством направлений, по которому его спектр уходит в бесконечность, и наличием в аннуляторном подмодуле подпространства $W$ функций, допускаюших определенные оценки снизу. Чтобы дать представление, насколько далеко можно продвинуться в одномерном случае по сравнению с многомерным, воспроизведем один результат из работы [35] (предложение 2.6).

Пусть $W$ - инвариантное подпространство в $\mathscr{H}(G)$, где $G$ - односвязная область в $\mathbb{C}$. Предположим, что $W$ допускает спектральньй синтез и аннуляторньй подмодуль $I$ подпространства содержит целую функцию вполне регулярного роста с сопряженной диаграммой $D \subset G$. Пусть $\Theta$ - множество предельных направлений спектра $\Lambda=\left\{\lambda_{i}, n_{i}\right\}$ подпространства $W$, т.е. множество точек на единичной окружности, являющихся пределами подпоследовательностей последовательности $\tau_{i}=\lambda_{i} /\left|\lambda_{i}\right|$, $i=1,2, \ldots, \Theta^{*}=\{\tau: \bar{\tau} \in \Theta\}$ - множество сопряженных направлений, $G \ominus D$ - геометрическая разность $G$ и $D$, т.е. множество $\{z: z+D \subset G\},(G \ominus D)_{0}-$ компонента этой разности, содержашая начало. Тогда любая функщия $f \in W$ допускает аналитическое продолжение в область

$$
G^{\prime}=\operatorname{conv}_{\Theta *}\left((G \ominus D)_{0}+D\right) .
$$


Здесь $\operatorname{conv}_{\Theta *}\left((G \ominus D)_{0}+D\right)$ обозначает $\Theta^{*}$-вьпуклую оболочку множества $\left((G \ominus D)_{0}+D\right)$ - пересечение всех полуплоскостей вида $\{z: \operatorname{Re}(z \bar{\tau})<p\}, \tau \in \Theta^{*}$, содержащих $\left((G \ominus D)_{0}+D\right)$.

Отметим, что из этого утверждения можно вывести цитированные вьше результаты, начиная с теоремы Адамара о степенных рядах с пропусками и кончая теоремой Леонтьева об аналитическом продолжении в вертикальную полосу.

\section{4. История задачи при $n>1$.}

В случае многих переменных задача аналитического продолжения мотивирована известньм фактом аналитичности решений многих классических уравнений математической физики.

Чтобы сформулировать известные результаты, введем ряд понятий. Направлени$e \mathcal{M ~ в ~} \mathbb{C}^{n}$ мы назьваем точку $\tau$ единичной сферы $S^{2 n-1} \subset \mathbb{C}^{n}$. Для $\tau \in S^{2 n-1} \tau$-nолупространством назьвается либо замкнутое полупространство вида

$$
\left\{z \in \mathbb{C}^{n}:(z, \tau) \leqslant a\right\},
$$

либо открытое полупространство вида

$$
\left\{z \in \mathbb{C}^{n}:(z, \tau)<a\right\},
$$

где $(z, \tau)=\operatorname{Re} \sum_{i=1}^{n} z_{i} \bar{\tau}_{i}, a \in \mathbb{R}$.

Пусть $\Theta$ - некоторое множество направлений, т.е. множество точек на $S^{2 n-1}$. Множество $M \subset \mathbb{C}^{n}$ называется $\Theta$-вылуклыци, если оно представляет собою пересечение некоторого семейства $\tau$-полупространств. Пересечение любого семейства $\Theta$-вьпуклых множеств - снова $\Theta$-вьпуклое множество. Поэтому имеет смысл понятие $\Theta$-выпуклой оболочки множества: это - пересечение всех $\Theta$-вьпуклых множеств, его содержащих. $\Theta$-вьпуклая оболочка множества $M$ обозначается conv $M$.

Пусть $\Lambda$ - некоторое множество в $\mathbb{C}^{n}$. Множеством предельных направлений множества $\Lambda$ называется множество пределов на сфере $S^{2 n-1}$ всевозможных последовательностей точек $z_{k} /\left|z_{k}\right|$, где $z_{k} \in \Lambda, z_{k} \rightarrow \infty$.

Пусть $D$-компакт в $\mathbb{C}^{n}$. Функция $H_{D}(\lambda)=\sup _{z \in D} \operatorname{Re}\langle z, \lambda\rangle$, где $\langle z, \lambda\rangle=\sum_{i=1}^{n} z_{i} \lambda_{i}$, называется опорной функиией (в смысле комплексного анализа) компакта $D$.

Пусть $\Omega$ - выпуклая область в $\mathbb{C}^{n}, S$ - линейный непрерьвный функционал на $\mathscr{H}(\Omega)$. Вьпуклый компакт $D$ назьвается определяющим для $S$, если для любой открытой окрестности $G$ компакта $D$ и любой целой функции $f \in \mathscr{H}\left(\mathbb{C}^{n}\right)$ вьполняется неравенство

$$
|\langle S, f\rangle| \leqslant \text { const } \cdot \sup _{z \in G}|f(z)| .
$$

Вьпукльй компакт $D \subset \mathbb{C}^{n}$ является определяющим для целой функции $\varphi$ экспоненциального типа, если для любого $\varepsilon>0$ существует константа $C_{\varepsilon}$ такая, что $|\varphi(\lambda)| \leqslant C_{\varepsilon} \exp \left(H_{D}(\lambda)+\varepsilon|\lambda|\right)$. Если $\varphi$ является характеристической функцией функционала $S$, т.е. $\varphi(\lambda)=\langle s, \exp \langle\lambda, z\rangle\rangle$, то по теореме Мартино-Эренпрайса [36; теорема 4.5.3] классы определяющих компактов для $S$ и $\varphi$ совпадают.

Пусть $P(D)$ - дифференциальный оператор в частных производных с постоянными коэффициентами. В 1967 г. П. Шапира [37] анонсировал, что каждое решение однородного уравнения

$$
P(D) u=0,
$$


голоморфное в вьпуклой области $\Omega \subset \mathbb{C}^{n}$, допускает аналитическое продолжение в $\Theta^{*}$-выпуклую оболочку области $\Omega$, т.е. в $\operatorname{conv} \Theta^{*} \Omega$, где $\Theta^{*}-$ множество направлений, сопряженных $\Theta$, а $\Theta$ - множество предельных направлений нулевого множества характеристического многочлена $P(\lambda)$ оператора $P(D)$. Это утверждение было доказано К. Кисельманом [38] в 1969 г.

Следует отметить, что возможность аналитического продолжения решений уравнения (6) в область $\operatorname{conv}_{\Theta} * \Omega$ следует из интегральных представлений для решений этого уравнения, связанных с фундаментальньм принципом Эренпрайса-Паламодова [39], [40]. А. Себбар [41] рассмотрел однородные уравнения бесконечного порядка с постоянными коэффициентами и с характеристической функцией минимального типа. При некоторых дополнительных условиях типа оценок снизу на характеристическую функцию каждое решение такого уравнения, голоморфное в вьпуклой области $\Omega \subset \mathbb{C}^{n}$, аналитически продолжается в $\operatorname{conv}_{\Theta} * \Omega$, где $\Theta-$ множество предельных направлений нулевого множества характеристической функщии. А. Мерил и Д. Струппа [42] рассмотрели более обшую ситуацию уравнения свертки

$$
S * f=0
$$

где $S$ - аналитический функционал с определяюшим компактом $D$. При весьма жестких ограничениях на характеристическую функцию $\varphi$ функционала $S$, включаюших полную регулярность ее роста, доказьвается, что всякое решение $f$ уравнения (7), голоморфное в области $D+\omega$, где $\omega$-вьпуклая область в $\mathbb{C}^{n}$, допускает аналитическое продолжение в область

$$
D+\operatorname{conv}_{\Theta} * \omega
$$

где $\Theta$ - множество предельных направлений нулевого множества функции $\varphi$.

Наиболее сильный результат в обсуждаемом направлении был получен А. С. Кривошеевым [43]. Воспроизвести формулировку этого результата в том виде, в каком она дана в [43], не представляется возможным, так как она не вписывается в контекст настояшей статьи. Мы придадим этому результату другую форму, доступную геометрическому восприятию. Нам понадобится понятие геометрической разности множеств $\Omega$ и $D$ в $\mathbb{C}^{n}$. Геометрической разностью множеств $\Omega$ и $D$, в обозначении $\Omega \ominus D$, называется множество $\left\{z \in \mathbb{C}^{n}: z+D \subset \Omega\right\}$. Если $\Omega$ не содержит ни одного сдвига $D$, то $\Omega \ominus D$ пусто. Если $\Omega$ - выпуклая область, а $D$ - компакт, принадлежаший $\Omega$, то геометрическая разность $\Omega \ominus D$ - вьпуклая область, содержащая начало координат.

Рассмотрим уравнение свертки (7) в вьпуклой области $\Omega$. Пусть $D$ - какой-либо определяющий компакт функщионала $S$, принадлежаший $\Omega, \varphi$-характеристическая функция функционала $S, H_{\varphi}(\lambda)$ - регуляризованньй индикатор $\varphi, H_{D}(\lambda)$ - опорная функция $D, \Theta$ - множество предельных направлений нулевого множества функции $\varphi$. Предполагаются выполненньми условия:

а) характеристическая функция $\varphi$ имеет вполне регулярный рост по множеству направлений $\Theta$;

б) $H_{\varphi}(\tau)=H_{D}(\tau)$ для всех $\tau \in \Theta$;

в) $H_{\Omega}(\tau)=H_{\Omega_{0}}(\tau)$ для всех $\tau \in \Theta$, где $H_{\Omega}(\lambda), H_{\Omega_{0}}(\lambda)$ - опорные функции соответственно $\Omega$ и $\Omega_{0}=(\Omega \ominus D)+D$. 
Тогда каждое решение $f \in \mathscr{H}(\Omega)$ уравнения свертки $(7)$ аналитически продолжается в $\operatorname{conv}_{\Theta^{*}} \Omega$.

Геометрический смысл условия в) состоит в том, что границы областей $\Omega$ и $\Omega_{0}$ совпадают в точках опоры $\tau$-полупространств при $\tau \in \Theta^{*}$.

5. Основное содержание работы.

В настоящей работе схема статьи [35] распространяется на многие переменные и $q>1$. Чтобы описать эту схему, нам потребуется специальная терминология.

Пусть $\Omega=\left\{\Omega_{1}, \ldots, \Omega_{q}\right\}$ - система областей в $\mathbb{C}^{n}, E-$ некоторое множество элементарных ехр- $q$-многочленов, инвариантное относительно операторов частного дифференцирования. Будем говорить, что инвариантное подпространство $W \subset \mathscr{H}(\Omega)$ порожсдено $E$, если $W$ совпадает с замыканием в топологии $\mathscr{H}(\Omega)$ линейной оболочки $E$. Очевидно, что $W$ - инвариантное подпространство, допускающее спектральньй синтез; обратно, всякое инвариантное подпространство, допускающее спектральный синтез, порождается некоторьм множеством элементарных ехр- $q$-многочленов.

Рассмотрим теперь две системы областей $\Omega=\left\{\Omega_{1}, \ldots, \Omega_{q}\right\}, \Omega^{\prime}=\left\{\Omega_{1}^{\prime}, \ldots, \Omega_{q}^{\prime}\right\}$ и два множества $E, E^{\prime}$ элементарных ехр-q-многочленов. Предположим, что $\Omega \subset \Omega^{\prime}$, т.е. $\Omega_{i} \subset \Omega_{i}^{\prime}, i=1, \ldots, q$, и $E^{\prime} \subset E$. Пусть $W$ - инвариантное подпространство в $\mathscr{H}(\Omega)$, порожденное $E, W^{\prime}$ - инвариантное подпространство в $\mathscr{H}\left(\Omega^{\prime}\right)$, порожденное $E^{\prime}$. Линейное непрерывное отображение

$$
p: W \rightarrow W^{\prime}
$$

называется проектором Дирихле (см. §6, п. 1), если оно обладает следующим свойством:

$$
p(e)=\left\{\begin{array}{l}
e, \text { если } e \in E^{\prime}, \\
0, \text { если } e \in E \backslash E^{\prime} .
\end{array}\right.
$$

Известным образом (§2, п. 2) пространства, сопряженные к $\mathscr{H}(\Omega), \mathscr{H}\left(\Omega^{\prime}\right)$, можно отождествить с пространствами $P, P^{\prime}$ целых $q$-функций экспоненциального типа. Эти пространства обладают структурой модулей над кольцом многочленов $\mathbb{C}\left[z_{1}, \ldots, z_{n}\right]$ и назьваются модулями, ассоциированными с $\Omega, \Omega^{\prime}$ соответственно. Инвариантньм подпространствам $W, W^{\prime}$ соответствуют их аннуляторные подмодули $I \subset P, I^{\prime} \subset P^{\prime}$ $(\S 2$, п. 2). Сушествование проектора Дирихле равносильно существованию так называемого мономорфизма Дирихле $(\S 7$, п. 1$)$ - специального линейного непрерывного отображения фактор-модуля $P^{\prime} / I^{\prime}$ в фактор-модуль $P / I$.

Вопрос о сушествовании мономорфизма Дирихле сводится к разрешимости следующей интерполяционной задачи: для каждого элемента $\varphi^{\prime} \in P^{\prime}$ найти элемент $\varphi \in P$ такой, что $\varphi \equiv \varphi^{\prime}(\bmod I(\lambda))$ для всех $\lambda \in \mathbb{C}^{n}(\S 7$, п. 1$)$, здесь $I(\lambda)$ - локальный подмодуль, порожденный $I$ в точке $\lambda(\S 2$, п. 2). В одномерном случае эта задача решалась с помощью интегрирования вдоль контуров, охватывающих спектральное множество инвариантного подпространства [36]; в многомерном случае эта задача решается специальным приемом, восходящим к K. Кисельману [38] и использующим оценку Л. Хёрмандера для неоднородного уравнения Коши-Римана (см. [36; теорема 4.4.2]). Реализация описанной вьше схемы приводит к общей теореме 10.1 о существовании проекторов Дирихле. Эта теорема имеет многочисленные следствия. В частности, с ее помощью мы получаем весьма общую теорему об аналитическом продолжении. Ниже мы сформулируем вариант этой теоремы, относяшийся к случаю $q=1$. 


\section{6. Теорема об аналитическом продолжении.}

Для формулировки теоремы потребуется ряд дополнительных понятий.

$1^{\circ}$. Пусть $\Gamma$ - замкнутое множество в $\mathbb{C}^{n}$, дополнение которого $\Gamma^{(c)}=\mathbb{C}^{n} \backslash \Gamma$ состоит не менее чем из двух компонент связности. Для компоненты связности $e \subset \Gamma^{(c)}$ обозначим через $E_{e}$ объединение всех компонент связности множества $\Gamma^{(c)}$, отличных от $е$. Определим на $\Gamma^{(c)}$ функцию $T_{\Gamma}(z)$, полагая

$$
T_{\Gamma}(z)=\operatorname{dist}\left(z, E_{e}\right)
$$

при $z \in e$.

Множество Г является окружением множества $\Lambda$, если существует разбиение $\Gamma^{(c)}$ на два открытых непустых множества $Z^{\prime}, Z^{\prime \prime}$, первое из которых содержит $\Lambda$. Ясно, что множества $Z^{\prime}, Z^{\prime \prime}$ не пересекаются и каждое из них представляет собою объединение некоторого семейства компонент связности множества $\Gamma^{(c)}$.

Понятие окружения в $\mathbb{C}^{n}$ играет ту же роль, что и понятие замкнутого контурав $\mathbb{C}$. При наличии оценок снизу вдоль окружения можно, используя оценки Хёрмандера, добиться того же эффекта, что и с помошью интеграла типа Коши в $\mathbb{C}$.

$2^{\circ}$. Пусть $A, B$ - вьпуклье множества в $\mathbb{C}^{n}$. Множество $M \subset \mathbb{C}^{n}$ назьвается $(A, B)$-выпукльлм, если оно обладает следующим свойством: если сдвиг $A+a$ множества $A$ на вектор $а$ принадлежит $M$, то и сдвиг $B+a$ также принадлежит $M$. Пересечение любого семейства $(A, B)$-вьпуклых множеств является $(A, B)$-выпуклым; поэтому для любого множества $M$ существует его $(A, B)$-вьпуклая оболочка - пересечение всех $(A, B)$-выпуклых множеств, его содержащих. Если множество $M$ не содержит ни одного сдвига множества $A$, то оно постулируется $(A, B)$-вьпуклым при любом $B ;(A, B)$-выпуклая оболочка такого множества совпадает с ним самим.

Понятия спектрального множества инвариантного подпространства и его аннуляторного подмодуля содержатся в $§ 2$.

ТЕОРЕМА ОБ АНАЛИТИЧЕСКОМ ПРОДОЛЖЕНИИ. Пусть $\Omega-$ область $\boldsymbol{в} \mathbb{C}^{n} ; W-$ инвариантное подпространство в $\mathscr{H}(\Omega)$, допускающее спектральньй синтез; $I$ - аннуляторный подмодуль подпространства $W, \Lambda$ - его спектральное множество; Г - замкнутое подмножсество в $\mathbb{C}^{n}$, окружающее $\Lambda$.

Предположим, что аннуляторный подмодуль I подпространства $W$ содержит функцию и такую, что выполняются следующие условия:

1)

$$
\varliminf_{|\lambda| \rightarrow \infty, \lambda \in \Gamma^{(c)}} \frac{\ln T_{\Gamma}(\lambda)}{|\lambda|} \geqslant 0 ;
$$

2) для некоторого выпуклого компакта $d \subset \mathbb{C}^{n}$ выполняется оценка снизу

$$
\ln |u(\lambda)| \geqslant H_{d}(\lambda)+o(|\lambda|), \quad \lambda \rightarrow \infty, \quad \lambda \in \Gamma
$$

3) для некоторого определяюшего компакта $D$ функции и выполняется включение

$$
d \subset D
$$


Тогда для любого выпуклого компакта $\omega \subset \mathbb{C}^{n}$ любая функция $f \in W$ аналитически продолжается в $(A, B)$-вьпуклую оболочку $\Omega^{\prime}$ области $\Omega$, где $A=D+\omega$, $B=\operatorname{conv}_{\Theta_{Z^{\prime} \cup \Gamma}^{*}}(d+\omega), \Theta_{Z^{\prime} \cup \Gamma}-$ предельное множество направлений множества $Z^{\prime} \cup \Gamma, \Theta_{Z^{\prime} \cup \Gamma}^{*}-$ множество сопряженных направлений.

Это утверждение является следствием общей теоремы о существовании проекторов Дирихле (теорема 10.1), относящейся к случаю $q \geqslant 1$.

Если аннуляторньй подмодуль $I$ подпространства $W$ содержит другую функцию $u$ и при этом вьполняются условия 1)-3) теоремы, может быть, с другими $\Gamma, D, d, \omega$, то элементы из $W$ будут допускать аналитическое продолжение в соответствуюшую $\left(A^{\prime}, B^{\prime}\right)$-вьпуклую оболочку, скажем $\Omega^{\prime \prime}$, отличную от $\Omega^{\prime}$. Тогда аналитическое продолжение будет осуществляться в объединение $\Omega^{\prime} \cup \Omega^{\prime \prime}$. Так что, варьируя $u, \Gamma, D, d$, $\omega$ допустимым образом, мы можем сушественно расширить область аналитического продолжения.

Заметим, что $(A, B)$-вьпуклая оболочка области $\Omega$ вместе с $A=D+\omega$ содержит и $B=\operatorname{conv}_{\Theta_{Z^{\prime} \cup \Gamma}^{*}}(d+\omega)$. Отсюда получаем

СлЕДСТВИЕ 1. При выполнении условий 1)-3) каждый әлемент $f \in W$ допускает аналитическое продолжение в $\operatorname{conv}_{\Theta_{Z^{\prime} \cup \Gamma}^{*}}(d+\omega)$.

Мы применим это следствие к подпространству $W \subset \mathscr{H}(\Omega)$ решений $f$ однородного уравнения свертки (7). Известно, что $W$ допускает спектральньй синтез [13] и что характеристическая функция $u(\lambda)=\langle S, \exp \langle\lambda, z\rangle\rangle$ этого уравнения принадлежит аннуляторному подмодулю $I$ подпространства $W$.

СЛЕДСТВИЕ 2. Пусть $\Omega=D+\omega$, где $D$ - какой-либо определяющий компакт функиионала $S, \omega$ - выпуклая область, и $\Theta$-множество предельньх направлений нулевого множества и. Предположим, что выполняются условия: а) характеристическая функция и имеет вполне регулярный рост по множеству направлений $\Theta$; б) $H_{u}(\tau)=H_{D}(\tau)$ для всех $\tau \in \Theta$.

Тогда каждое решение $f \in \mathscr{H}(\Omega)$ уравнения свертки (7) аналитически продолжается в $\operatorname{conv}_{\Theta^{*}} \Omega$.

ДоКАЗАТЕЛЬство. При выполнении условий а), б) можно построить окружение Г множества $\Lambda$ (это фактически сделано в [43]) такое, что множество $\Theta_{Z^{\prime} \cup \Gamma}$ совпадает с $\Theta$ и выполняется оценка $(9)$ с $d=D$. По следствию 1 любое решение $f \in \mathscr{H}(\Omega)$ уравнения свертки (7) аналитически продолжается в $\operatorname{conv}_{\Theta} * \Omega$.

Все перечисленные вьше результаты, относяшиеся к уравнению свертки при $n>1$, следуют из этой теоремы. Результаты П. Шапиры [37], К. Кисельмана [38], А. Себбара [41] получаются при $D=\{0\}$. Утверждение А. Мерила и Д. Струпшы [42] следует из включения

$$
D+\operatorname{conv}_{\Theta} * \omega \subset \operatorname{conv}_{\Theta} *(D+\omega) .
$$

Утверждение А. С. Кривошеева [43] выводится с помошюю следуюшего рассуждения. Пусть вьполняются условия а)-в) П. 4. Рассмотрим решение $f$ уравнения свертки (7), голоморфное в $\Omega$. Так как $\Omega_{0} \subset \Omega$, то тем более $f$ голоморфно в $\Omega_{0}$. Но область $\Omega_{0}$ имеет вид, требуемьй следствием 2 , именно: $\Omega_{0}=D+\omega$, где $\omega=\Omega \ominus D$. Поэтому $f$ аналитически продолжается в conv ${ }^{*} \Omega_{0}$. Чтобы завершить рассуждение, достаточно заметить, что при условии в) $\operatorname{conv}_{\Theta} * \Omega=\operatorname{conv}_{\Theta} * \Omega_{0}$. 


\section{§ 2. Спектральный синтез и обильные подмодули}

\section{1. Обозначения.}

Объектами исследования настоящей работы являются инвариантные подпространства, допускаюшие спектральньй синтез. Если $W$ - такое подпространство в $\mathscr{H}(G)$, где $G$ - открытое множество в $\mathbb{C}^{n}$, то каждый элемент из $W$ аппроксимируется равномерно внутри $G$ полиномами Дирихле и, следовательно, обычными полиномами. Это значит, что $W$ вложено в подпространство $H(G)$ пространства $\mathscr{H}(G)$, состоящее из функций, аппроксимируемых полиномами. Наделив последнее топологией, индуцированной из $\mathscr{H}(G)$, мы можем рассматривать его как самостоятельное локально-вьпуклое пространство, а $W$ - как вложенное в него. Такой подход позволяет избежать сложностей, связанных со строением открытых множеств, в которых голоморфные функции апшроксимируются многочленами (множеств Рунге). Хорошо известно, что $\mathscr{H}(G)$ является пространством типа $\left(M^{*}\right)[44]$. Как замкнутое подпространство этого пространства $H(G)$ также является пространством типа $\left(M^{*}\right)$. Назовем $H(G)$ пространством, ассочиированным с $G$.

Пусть $\Omega=\left(\Omega_{1}, \ldots, \Omega_{q}\right)$ - упорядоченная система открытых множеств в $\mathbb{C}^{n}$;

$$
H(\Omega)=H\left(\Omega_{1}\right) \times \cdots \times H\left(\Omega_{q}\right)
$$

- топологическое произведение пространств $H\left(\Omega_{1}\right), \ldots, H\left(\Omega_{q}\right)$, ассоциированных с $\Omega_{1}, \ldots, \Omega_{q}$ соответственно. Для сокрашения формулировок удобно ввести следующую терминологию. У Уорядоченную систему $K=\left(K_{1}, \ldots, K_{q}\right)$ множеств $K_{1}, \ldots$, $K_{q} \subset \mathbb{C}^{n}$ будем назьвать $q$-множеством. $K$ - открытое $q$-множество, если все $K_{i}-$ открытые множества; $K$ - вьпуклая $q$-область, если все $K_{i}$ - вьпуклые области; $K-$ вьпукльй $q$-компакт, если все $K_{i}$ - выпуклые компакты. По отношению к $q$-множествам теоретико-множественные отношения включения и операции объединения и пересечения имеют покомпонентный смысл. Так, включение $K \subset K^{\prime}$ для $q$-множеств $K=\left(K_{1}, \ldots, K_{q}\right), K^{\prime}=\left(K_{1}^{\prime}, \ldots, K_{q}^{\prime}\right)$ равносильно $q$ включениям $K_{i} \subset K_{i}^{\prime}$, $i=1, \ldots, q$. Объединение $K=\bigcup_{\alpha} K^{\alpha}$ равносильно $K_{i}=\bigcup_{\alpha} K_{i}^{\alpha}, i=1, \ldots, q$. Напомним, что арифметическая сумма $A+B$ множеств $A, B \subset \mathbb{C}^{n}$ представляет собою множество всех сумм $a+b$, где $a \in A, b \in B$. В этой статье мы будем иметь дело с арифметическими суммами вида $K+A$, где $K=\left(K_{1}, \ldots, K_{q}\right)$ - q-множество, а $A$ - множество из $\mathbb{C}^{n}$; при этом под суммой $K+A$ мы подразумеваем $q$-множество $\left(K_{1}+A, \ldots, K_{q}+A\right)$, где $K_{i}+A-$ арифметическая сумма $K_{i}$ и $A$.

Пространство $H(\Omega)$, введенное по формуле (1), будем кратко обозначать $H$ и называть пространством, ассоциированнылм с открытым q-множеством $\Omega$. Как топологическое произведение пространств $H\left(\Omega_{1}\right), \ldots, H\left(\Omega_{q}\right)$ пространство $H$ наследует их свойства; в частности, оно является пространством типа $\left(M^{*}\right)$. Элементами $H$ являются векторы $f=\left(f_{1}, \ldots, f_{q}\right)$ с компонентами $f_{i} \in H\left(\Omega_{i}\right)$. Опишем сходимость в топологии $H$. Пусть $K=\left(K_{1}, \ldots, K_{q}\right)-q$-компакт в $\Omega$. Последовательность элементов

$$
f^{(k)}=\left(f_{1}^{(k)}, \ldots, f_{q}^{(k)}\right), \quad k=1,2, \ldots,
$$

сходится к элементу $f=\left(f_{1}, \ldots, f_{q}\right)$ равномерно на $K$, если $f_{i}^{(k)} \rightarrow f_{i}$ равномерно на $K_{i}$ для $i=1, \ldots, q$. Сходимость последовательности $(2)$ к элементу $f$ в топологии $H$ равносильна сходимости этой последовательности к элементу $f$ равномерно внутри $\Omega$, т.е. равномерно на каждом $q$-компакте $K \subset \Omega$. 
Чтобы указать зависимость компонент элемента $f=\left(f_{1}, \ldots, f_{q}\right)$ от соответствуюших переменных, правильно было бы использовать запись $\left(f_{1}\left(z^{(1)}\right), \ldots, f_{q}\left(z^{(q)}\right)\right)$, где векторные переменные $z^{(1)}=\left(z_{1}^{(1)}, \ldots, z_{n}^{(1)}\right), \ldots, z^{(q)}=\left(z_{1}^{(q)}, \ldots, z_{n}^{(q)}\right)$ изменяются в множествах $\Omega_{1}, \ldots, \Omega_{q}$ соответственно. Однако более удобна символическая запись

$$
f(z)=\left(f_{1}(z), \ldots, f_{q}(z)\right)
$$

где неявно предполагается, что в каждой компоненте переменная $z=\left(z_{1}, \ldots, z_{n}\right)$ меняется независимо от переменных в других компонентах. Продемонстрируем удобство такой символики. Будем $q$-одночлен, т.е. элемент вида

$$
\left(a_{1}\left(z^{(1)}\right)^{\alpha}, \ldots, a_{q}\left(z^{(q)}\right)^{\alpha}\right)
$$

где $a_{1}, \ldots, a_{q}$ - комплексные числа, $\alpha=\left(\alpha_{1}, \ldots, \alpha_{n}\right)$ - мультииндекс, $\left(z^{(i)}\right)^{\alpha}=$ $\left(z_{1}^{(i)}\right)^{\alpha_{1}} \cdots\left(z_{n}^{(i)}\right)^{\alpha_{n}}$, записывать символически в виде $a z^{\alpha}$, где $a=\left(a_{1}, \ldots, a_{q}\right)-$ вектор-столбец, умножение монома $z^{\alpha}=z_{1}^{\alpha_{1}} \cdots z_{n}^{\alpha_{n}}$ на вектор $a$ производится покомпонентно. q-многочлен стелени $m$, т.е. элемент вида $\left(p_{1}\left(z^{(1)}\right), \ldots, p_{q}\left(z^{(q)}\right)\right)$, где $p_{i}\left(z^{(i)}\right)$ - многочлен от переменных $z_{1}^{(i)}, \ldots, z_{n}^{(i)}$ и $\max _{i} \operatorname{deg} p_{i}=m$, допускает запись в виде суммы $q$-одночленов

$$
\sum_{|\alpha|=0}^{m} a_{\alpha} z^{\alpha},
$$

где $|\alpha|=\alpha_{1}+\cdots+\alpha_{n}, a_{\alpha}$ - векторы-столбцы. Наконец, әлементарный ехр-q-многочлен степени $m$, т.е. элемент вида

$$
\left(p_{1}\left(z^{(1)}\right) \exp \left\langle\lambda, z^{(1)}\right\rangle, \ldots, p_{q}\left(z^{(q)}\right) \exp \left\langle\lambda, z^{(q)}\right\rangle\right),
$$

где $p_{i}-$ многочлен, $\max _{i} \operatorname{deg} p_{i}=m$, допускает символическую запись

$$
p(z) \exp \langle\lambda, z\rangle
$$

где $p(z)-q$-многочлен степени $m$ и экспонента $\exp \langle\lambda, z\rangle$ умножается на $q$-многочлен $p(z)$ покомпонентно. Конечную сумму элементарных ехр- $q$-многочленов будем называть просто ехр- $q$-многочленом или полиномом Дирихле.

\section{2. Аннуляторный подмодуль.}

Задача спектрального синтеза сводится к задаче локального описания с помошюю принципа двойственности, восходящего к Л. Эренпрайсу [11]. Сначала рассмотрим случай $q=1$. Пусть $\Omega$ - открытое множество в $\mathbb{C}^{n}, H$ - пространство, ассоциированное с $\Omega, H^{*}$ - сильное сопряженное к $H$. Каждому линейному непрерывному функционалу $S \in H^{*}$ поставим в соответствие его характеристическую функцию $\varphi(\lambda)=\langle S, \exp \langle\lambda, z\rangle\rangle$. Когда $S$ пробегает $H^{*}$, его характеристическая функция $\varphi$ пробегает некоторьй класс целых функций $P$. Этот класс обладает структурой модуля над кольцом многочленов и наделяется топологией, индуцированной из $H^{*}$ отображением $T: S \mapsto \varphi(\lambda)=\langle S, \exp \langle\lambda, z\rangle\rangle$. Будем называть $P$ модулем, ассоциированнылм 
с открытым множеством $\Omega$, а отображение $T: H^{*} \rightarrow P-$ каноническим. Поскольку система всех одночленов, а значит, и система всех экспонент, полны в $H$, ядро отображения $T: H^{*} \rightarrow P$ тривиально. Отсюда следует, что отображение $T$ - топологический изоморфизм. Пространство Р как изоморфное пространству, сопряженному к пространству типа $\left(M^{*}\right)$, является пространством типа $\left(L N^{*}\right)$ и, значит, является бочечньм, борнологическим, рефлексивным [45; гл. ХI].

Теперь рассмотрим случай $q>1$. Пусть $H$ - пространство, определенное по формуле (1). Пространство $H^{*}$, сопряженное к $H$, представляется в виде топологического произведения

$$
H^{*}=H_{1}^{*} \times \cdots \times H_{q}^{*}
$$

где $H_{i}^{*}$ - пространство, сопряженное к $H\left(\Omega_{i}\right)$. Линейный непрерывньй функционал $S \in H^{*}$ представляется в виде вектора $S=\left(S_{1}, \ldots, S_{q}\right), S_{i} \in H_{i}^{*}$. Действие функционала $S$ на элемент $f=\left(f_{1}, \ldots, f_{q}\right) \in H$ осушествляется по формуле

$$
\langle S, f\rangle=\sum_{i=1}^{q}\left\langle S_{i}, f_{i}\right\rangle .
$$

Сопоставим функционалу $S=\left(S_{1}, \ldots, S_{q}\right)$ его характеристический элемент

$$
\varphi(\lambda)=\left(\varphi_{1}(\lambda), \ldots, \varphi_{q}(\lambda)\right)
$$

где $\varphi_{i}(\lambda)=\left\langle S_{i}, \exp \langle\lambda, z\rangle\right\rangle$. Когда $S$ пробегает $H^{*}$, его характеристический элемент $\varphi(\lambda)=\left(\varphi_{1}(\lambda), \ldots, \varphi_{q}(\lambda)\right)$ пробегает класс $P$ целых $q$-функций экспоненциального типа. Класс $P$ обладает структурой линейного пространства над полем $\mathbb{C}$ и выдерживает умножение (покомпонентное) на многочлены.

Обозначим через $T$ операцию перехода от функционала $S$ к его характеристическому элементу. $T$ является линейньм отображением $H^{*}$ на $P$. Наделим $P$ локально-вьпуклой топологией, индуцированной из $H^{*}$ отображением $T$. Легко видеть, что $P$ представляет собою топологическое произведение

$$
P=P_{1} \times \cdots \times P_{q}
$$

где $P_{i}$ - модуль, ассоциированньй с $\Omega_{i}$. Произведение $P$ наследует свойства пространств $P_{i}$ : оно является пространством типа $\left(L N^{*}\right)$, бочечным, борнологическим, рефлексивньм. Операция умножения на многочлен непрерывна в топологии $P$. Это означает, что $P$ обладает структурой топологического модуля над кольцом $\mathbb{C}[\lambda]=$ $\mathbb{C}\left[\lambda_{1}, \ldots, \lambda_{n}\right]$. Будем $P$ называть модулем, ассоциированным с открытымм q-множеством $\Omega$, а отображение $T: H^{*} \rightarrow P$ - каноническим. Отображение $T$ является топологическим изоморфизмом. Это следует из того, что таковыми являются канонические отображения $T_{i}: H_{i}^{*} \rightarrow P_{i}$. Подмодулем $\mathbb{C}[\lambda]$-модуля $P$ назьвается подмножество $I \subset P$, обладающее свойствами:

1) $\varphi_{1}, \varphi_{2} \in I \Longrightarrow \varphi_{1}+\varphi_{2} \in I$

2) $\varphi \in I, p \in \mathbb{C}[\lambda] \Longrightarrow p \varphi \in I$. 
Существует связь между замкнутьми подмодулями $I \subset P$ и инвариантными подпространствами $W \subset H$. Эта связь устанавливается следуюшим образом. Рассматривается подпространство $W^{0}$ в $H^{*}$, ортогональное $W$ :

$$
W^{0}=\left\{S \in H^{*}:\langle S, f\rangle=0 \forall f \in W\right\} .
$$

В силу рефлексивности $H$ подпространство $W^{0}$ замкнуто в $H^{*}$. Стандартньм образом показывается, что образ $I=T\left(W^{0}\right)$ обладает структурой замкнутого подмодуля в $P$. Обратно, в силу того, что каноническое отображение $T: H^{*} \rightarrow P$ - топологический изоморфизм, каждому замкнутому подмодулю $I \subset P$ соответствует инвариантное подпространство $W \subset H$, связанное с $I$ соотношением $I=T\left(W^{0}\right)$. Подмодуль $I$ назьвается аннуляторнылм подмодулем инвариантного подпространства $W$ и обозначается An $W$. Соотношение $I=\operatorname{An} W$ устанавливает взаимно однозначное соответствие между совокупностью $\{W\}$ всех инвариантных подпространств в $Н$ и совокупностью $\{I\}$ всех замкнутых подмодулей в $P$. Инвариантньм подпространствам, допускаюшим спектральный синтез, соответствуют замкнутые подмодули, обладаюшие специальным свойством. Чтобы описать это свойство, введем понятие локального подмодуля $I(\lambda)$, порождаемого подмодулем $I$ в точке $\lambda \in \mathbb{C}^{n}$. Обозначим через $O(\lambda)$ кольцо ростков функций, аналитических в точке $\lambda$, а через $O^{q}(\lambda)$ - совокупность ростков $q$-функций, аналитических в точке $\lambda$. Очевидно, что $O^{q}(\lambda)$ обладает структурой модуля над кольцом $O(\lambda)$. Фиксированный подмодуль $I \subset P$ порождает в каждой точке $\lambda \in \mathbb{C}^{n}$ локальный подмодуль $I(\lambda)$; последний состоит из всевозможных конечных линейных комбинаций элементов из $I$ с коэффициентами из $O(\lambda)$ :

$$
\sum c_{i} u^{(i)}, \quad u^{(i)} \in I, \quad c_{i} \in O(\lambda)
$$

Элемент $f$ из $P$ принадлежит $I$ локально, в записи $f \underset{\text { оос }}{\in} I$, если $f \in I(\lambda) \forall \lambda \in \mathbb{C}^{n}$. Подмодуль $I$ обильный, если справедлива импликация

$$
f \in P, \quad f \underset{\text { loc }}{\in} I \Longrightarrow f \in I .
$$

Термин “обильньй подмодуль" проистекает из того обстоятельства, что среди подмодулей с одинаковым локальным поведением обильные подмодули имеют наибольший запас элементов; иными словами, если $I$ - обильный подмодуль в $P$, а $I^{\prime}$ - другой подмодуль в $P$ с тем же набором локальных подмодулей, т.е. $I^{\prime}(\lambda)=I(\lambda) \forall \lambda \in \mathbb{C}^{n}$, то $I^{\prime} \subset I$. Очевидно, что обильные подмодули и только они допускают локальное описание, т.е. полностью восстанавливаются по набору $\{I(\lambda)\}, \lambda \in \mathbb{C}^{n}$, своих локальных подмодулей.

\section{3. Спектр.}

В пространстве $H$ действуют $n$ операторов частного дифференцирования

$$
D_{i}:\left(f_{1}, \ldots, f_{q}\right) \rightarrow\left(\frac{\partial}{\partial z_{i}} f_{1}, \ldots, \frac{\partial}{\partial z_{i}} f_{q}\right), \quad i=1, \ldots, n .
$$

Элемент $e \in H$ назьвается корневым әлементом системы операторов $D_{1}, \ldots, D_{n}$, отвечаюшим вектору собственных значений $\lambda=\left(\lambda_{1}, \ldots, \lambda_{n}\right)$, если для некоторого положительного целого числа $m$ элемент $е$ удовлетворяет системе уравнений

$$
\left(D_{1}-\lambda_{1} \mathrm{E}\right)^{m} e=0, \ldots,\left(D_{n}-\lambda_{n} \mathrm{E}\right)^{m} e=0
$$


где Е - тождественный оператор. Из теории дифференциальных уравнений известно, что целые решения системы (3) представляют собою ехр- $q$-многочлены степени, меньшей $m$, с показателем $\lambda$. Описание запаса элементарных exp- $q$-многочленов, содержашихся в инвариантном подпространстве $W \subset H$, осушествляется с помошю спектра этого подпространства. Обозначим $\mathbb{C}^{q}[\xi]=\mathbb{C}^{q}\left[\xi_{1}, \ldots, \xi_{n}\right]$ множество $q$-многочленов переменных $\xi_{1}, \ldots, \xi_{n}$. Легко видеть, что $\mathbb{C}^{q}[\xi]$ выдерживает умножение (покомпонентное) на многочлены $p(\xi)$. Поэтому можем рассматривать $\mathbb{C}^{q}[\xi]$ как модуль над кольцом многочленов $\mathbb{C}[\xi]$. Фиксируем точку $\lambda \in \mathbb{C}^{n}$. Локальный спектр $W$ в точке $\lambda$ определяется как множество

$$
\operatorname{Sp}_{\lambda} W:=\left\{p \in \mathbb{C}^{q}[\xi]: p(z) \exp \langle\lambda, z\rangle \in W\right\} .
$$

Локальный спектр обладает следующими свойствами:

$1^{\circ} p_{1}, p_{2} \in \mathrm{Sp}_{\lambda} W, c_{1}, c_{2} \in \mathbb{C} \Longrightarrow c_{1} p_{1}+c_{2} p_{2} \in \operatorname{Sp}_{\lambda} W$

$2^{\circ} W \subset W^{\prime} \Longrightarrow \operatorname{Sp}_{\lambda} W \subset \operatorname{Sp}_{\lambda} W^{\prime}$

$3^{\circ} p \in \operatorname{Sp}_{\lambda} W \Longrightarrow p^{(\alpha)} \in \operatorname{Sp}_{\lambda} W$, где $\alpha=\left(\alpha_{1}, \ldots, \alpha_{n}\right)$ - мультииндекс и $p^{(\alpha)}(z)=$ $\frac{\partial^{\alpha_{1}+\cdots+\alpha_{n}}}{\partial^{\alpha_{1}} z_{1} \cdots \partial^{\alpha_{n}} z_{n}} p(z)$

$C n е к т p ~ W$ определяется как дизъюнктное объединение локальных спектров

$$
\operatorname{Sp} W:=\bigsqcup_{\lambda \in \mathbb{C}^{n}} \operatorname{Sp}_{\lambda} W
$$

Спектр $W$ можно представить как совокупность пар $(\lambda, p), \lambda \in \mathbb{C}^{n}, p \in \mathbb{C}^{q}[\xi]$, для которых $p(z) \exp \langle\lambda, z\rangle \in W$.

Точка $\lambda \in \mathbb{C}^{n}$ назьвается спектральной точкой инвариантного подпространства $W$, если $\operatorname{Sp}_{\lambda} W \neq\{0\}$. Множество всех спектральных точек подпространства $W$ образует спектральное множество этого подпространства ${ }^{1}$.

\section{4. Дивизор.}

\section{1. Локальный дивизор. Рассмотрим $q$-многочлен}

$$
p(\xi)=\left(p_{1}(\xi), \ldots, p_{q}(\xi)\right) \in \mathbb{C}^{q}[\xi] .
$$

Этому $q$-многочлену соответствует дифференциальньй $q$-оператор $p(D)=\left(p_{1}(D)\right.$, $\left.\ldots, p_{q}(D)\right)$, который $q$-функщию $\varphi=\left(\varphi_{1}, \ldots, \varphi_{q}\right)$, голоморфную в области $U \subset \mathbb{C}^{n}$, переводит в скалярную функцию $\psi$, голоморфную в той же области, по правилу

$$
\psi=\sum_{i=1}^{q} p_{i}(D) \varphi_{i} .
$$

Стандартным образом действие оператора $p(D)$ распространяется на ростки $q$-функций, аналитических в точке $\lambda$. Рассмотрим подмодуль $I \subset O^{q}(\lambda)$. Дивизор подмодуля $I$, в записи $\operatorname{Div} I,-$ это множество $q$-многочленов:

$$
\operatorname{Div} I:=\left\{p \in \mathbb{C}^{q}[\xi]:\left.p(D) u\right|_{\lambda}=0 \forall u \in I\right\} .
$$

В статье [15] установлены следуюшие свойства дивизора.

Свойство 1. Если $p_{1}, p_{2} \in \operatorname{Div} I, a_{1}, a_{2} \in \mathbb{C}, m o a_{1} p_{1}+a_{2} p_{2} \in \operatorname{Div} I$.

\footnotetext{
${ }^{1}$ В работе [14] спектральное множество называется алгебраическим спектром.
} 


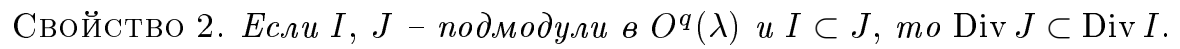

Свойство 3. Если $p \in \operatorname{Div} I$, mo $p^{\alpha} \in \operatorname{Div} I$, әде $\alpha=\left(\alpha_{1}, \ldots, \alpha_{n}\right)-$ мультииндeкc, $p^{(\alpha)}=\frac{\partial^{\alpha_{1}+\cdots+\alpha_{n}}}{\partial^{\alpha_{1}} z_{1} \cdots \partial^{\alpha_{n}} z_{n}} p$.

Свойство 4. Div $I \cup \operatorname{Div} J \subset \operatorname{Div}(I \cap J)$.

Для подмодулей $I, J$ символом $I \vee J$ обозначим подмодуль в $O^{q}(\lambda)$, порожденный $I$ и $J$.

Свойство 5. Справедливо соотношение

$$
\operatorname{Div}(I \vee J)=\operatorname{Div} I \cap \operatorname{Div} J .
$$

Свойство 6. Если $\operatorname{Div} I=\operatorname{Div} J, m o I=J$.

Свойство 7. Если Div $J \supset \operatorname{Div} I, m o J \subset I$.

Для произвольного множества $I \subset O^{q}(\lambda)$ дивизор $I$ определяется как дивизор подмодуля, порожденного $I$ в $O^{q}(\lambda)$; в частности, дивизор элемента $u \in O^{q}(\lambda)$, в обозначении $\operatorname{Div} u$, есть дивизор главного подмодуля, порожденного $u$ в $O^{q}(\lambda)$.

Свойство 8. Пусть $I-$ подмодуль в $O^{q}(\lambda), u \in O^{q}(\lambda)$. Ecли Div $u \supset \operatorname{Div} I, m o$ $u \in I$.

Пусть $I, J$ - множества в $O^{q}(\lambda)$. Множество $J$ зависит от множества $I$ (над кольцом $O(\lambda)$ ), если каждый элемент $v \in J$ представляется в виде конечной линейной комбинации

$$
v=\sum c_{i} u^{(i)}, \quad u^{(i)} \in I, \quad c_{i} \in O(\lambda) .
$$

Свойство 9. Для того чтобы множество J зависело от множества I, необходимо и достаточно, чтобь $\operatorname{Div} J \supset \operatorname{Div} I$.

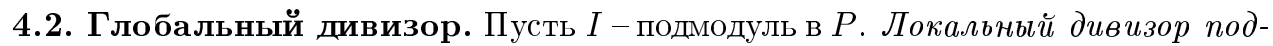
модуля $I$ в точке $\lambda$, в записи $\operatorname{Div}_{\lambda} I$, определяется как дивизор локального подмодуля $I(\lambda)$, порожденного $I$ в точке $\lambda: \operatorname{Div}_{\lambda} I:=\operatorname{Div} I(\lambda)$.

Дивизор $I$ есть дизъюнктное объединение локальных дивизоров

$$
\operatorname{Div} I:=\bigsqcup_{\lambda \in \mathbb{C}^{n}} \operatorname{Div}_{\lambda} I .
$$

В работе [15] доказано

ПРЕДЛОЖЕНИЕ 2.1. Пусть $W$ - инвариантное подпространство в $H, I=$ An $W$. Тогда $\operatorname{Sp} W=\operatorname{Div} I$ или, более точно,

$$
\operatorname{Sp}_{\lambda} W=\operatorname{Div}_{\lambda} I \quad \forall \lambda \in \mathbb{C}^{n} .
$$

Доказательство основано на следуюшем соотношении: для любого линейного непрерьвного функционала $S \in H^{*}$ и его характеристического элемента $\varphi$ справедливо соотношение

$$
\left.p(D) \varphi\right|_{\lambda}=\langle S, p(z) \exp \langle\lambda, z\rangle\rangle .
$$


ТЕОРема ДВОЙСТВеННОСТИ [14], [15]. Для того чтобы инвариантное подпространство $W \subset H$ допускало спектральный синтез, необходимо и достаточно, чтобь аннуляторный подмодуль $I=\operatorname{An} W$ этого подпространства был обильHbl.M.

\section{5. Нулевое множество подмодуля.}

Система элементов $u^{(1)}, \ldots, u^{(k)} \in O^{q}(\lambda)$ независима над кольцом $O(\lambda)$, если

$$
\sum c_{i} u^{(i)}=0, \quad c_{i} \in O(\lambda) \Longrightarrow c_{i}=0
$$

Если $J$ - подмодуль в $O^{q}(\lambda)$, то ранг $J$, в записи $\operatorname{rank} J,-$ максимальное число элементов в независимых системах $u^{(1)}, \ldots, u^{(k)} \in J$. Так же, как и в случае $n=1$ (см. $[46 ; \S 1])$, устанавливается, что $\operatorname{rank} J \leqslant q$.

Пусть $I$ - подмодуль в $P$. Нетрудно проверить, что ранг локального подмодуля $I(\lambda)$ не зависит от $\lambda$. Это число назьвается рангом $I$ и обозначается $\operatorname{rank} I$. Система элементов $u^{(1)}, \ldots, u^{(k)} \in P$ называется независимой, если она независима хотя бы над одним кольцом $O(\lambda)$. Ранг $I$ равен максимальному числу элементов в независимых системах $u^{(1)}, \ldots, u^{(k)} \in I$. Будем представлять элементы из $P$ как векторы-столбцы. Обозначим $\left(u^{(1)} \ldots u^{(k)}\right), k \leqslant q$, матрицу, составленную из векторов-столбцов $u^{(1)}, \ldots, u^{(k)}$. Рассмотрим минор

$$
\left[\begin{array}{ccc}
u^{(1)} & \ldots & u^{(k)} \\
i_{1} & \ldots & i_{k}
\end{array}\right]
$$

этой матрицы, полученньй выделением строк с номерами $i_{1}, \ldots, i_{k}, 1 \leqslant i_{1}<\ldots<$ $i_{k} \leqslant q$. Независимость системы $u^{(1)}, \ldots, u^{(k)}$ равносильна тому, что ранг матрищы $\left(u^{(1)} \ldots u^{(k)}\right)$ равен $k$, т.е. среди определителей вида (6) хотя бы один отличен от тождественного нуля.

В настоящей статье мы будем рассматривать только подмодули полного ранга $k=q$. Для таких подмодулей определим понятие нулевого множества. Пусть $I-$ подмодуль в $P$ полного ранга $q$. Рассмотрим всевозможные определители

$$
\Delta=\left|\begin{array}{ccc}
u_{1}^{(1)} & \ldots & u_{1}^{(q)} \\
u_{q}^{(1)} & \ldots & u_{q}^{(q)}
\end{array}\right|,
$$

где $u^{(1)}=\left(u_{1}^{(1)}, \ldots, u_{q}^{(1)}\right), \ldots, u^{(q)}=\left(u_{1}^{(q)}, \ldots, u_{q}^{(q)}\right)$ - элементы из $I$. Каждый определитель $\Delta$ представляет собою целую функцию. Точка $\lambda \in \mathbb{C}^{n}$ назьвается нулем подмодуля $I$, если все определители вида (7) обрашаются в нуль в точке $\lambda$. Совокупность $N$ всех нулей подмодуля $I$ образует его нулевое множсество. Нулевое множество $N$ является аналитическим в классическом смысле: для каждой точки $\lambda \in N$ сушествует открытая окрестность $Y$ этой точки такая, что $N \cap Y$ представляется как нулевое множество конечного числа аналитических функций. Это следует из известного факта нётеровости $[47 ; \S 1]$ любого локального подмодуля (в рассматриваемом случае - подмодуля, порожденного функциями, представленными в виде определителя (7)). В случае $q=1$ определители (7) совпадают с элементами $u \in I$ и $N$ совпадает с нулевым множеством подмодуля $I$ в прямом смысле. 
ПРЕДЛОЖЕНИЕ 2.2. Пусть $\operatorname{rank} I=q$. Для того чтобы локальный подмодуль $I(\lambda)$, порождаемый $I$ в точке $\lambda$, был нетривиальнылм, т.е. $I(\lambda) \neq O^{q}(\lambda)$, необходимо и достаточно, чтобы $\lambda$ было нулем подмодуля $I$.

ДокАЗАТЕЛЬСТво. Предложение эквивалентно элементарному утверждению: для того чтобы $I=O^{q}(\lambda)$, необходимо и достаточно, чтобы $\lambda$ не было нулем $I$.

ПРЕДЛОЖЕНИЕ 2.3. Спектральное множество инвариантного подпространства совпадает с нулевым множеством его аннуляторного подмодуля.

ДокАЗАТЕЛЬСТво. Пусть $\Lambda$ - спектральное множество инвариантного подпространства $W, N$ - нулевое множество его аннуляторного подмодуля $I$. Пусть $\lambda \in \Lambda$. Тогда $\operatorname{Sp}_{\lambda} W \neq\{0\}$. В силу соотношения (4) $\operatorname{Div}_{\lambda} I \neq\{0\}$. По свойству 7 дивизора отсюда следует, что $I(\lambda) \neq O^{q}(\lambda)$. По предложению 2.2 заключаем, что $\lambda \in N$. Таким образом, доказано, что $\Lambda \subset N$. Пусть теперь $\lambda \in N$. Согласно предложению 2.2 $I(\lambda) \neq O^{q}(\lambda)$. По свойству 7 дивизора $\operatorname{Div}_{\lambda} I \neq\{0\}$. В силу соотношения (4) будет $\mathrm{Sp}_{\lambda} W \neq\{0\}$, а это означает, что $\lambda \in \Lambda$. Таким образом, доказано, что $N \subset \Lambda$. Из вьшеизложенного следует $N=\Lambda$.

6. Квазиспектральное и квазинулевое множества.

Пусть $W$ - инвариантное подпространство в $H=H(\Omega)$, допускаюшее спектральный синтез; $\Lambda$ - спектральное множество подпространства $W, \Lambda^{\prime} \subset \Lambda$. Обозначим $W^{\prime}$ замькание в топологии $H(\Omega)$ множества линейных комбинаций ехр- $q$-многочленов из $W$ с показателями $\lambda \in \Lambda^{\prime}$. Будем $\Lambda^{\prime}$ называть квазиспектральнылм множеством инвариантного подпространства $W$, если $W^{\prime}=W$.

Пусть $I$ - обильньй подмодуль в $P, N$ - нулевое множество $I$. Множество $N^{\prime} \subset N$ называется квазинулевым множеством подмодуля $I$, если выполняется импликация

$$
f \in P, \quad f \in I(\lambda) \forall \lambda \in \Lambda^{\prime} \Longrightarrow f \in I .
$$

ПРЕДЛОЖЕНИЕ 2.4. Пусть I - аннуляторный подмодуль инвариантного подпространства $W$, допускающего спектральный синтез. Для того чтобь множество $\Lambda^{\prime}$ било квазиспектральным множеством подпространства $W$, необходимо и достаточно, чтобы $\Lambda^{\prime}$ было квазинулевым множеством его аннуляторного подмодуля.

ДокАЗАТЕЛЬство. Необходимость. Пусть $\Lambda$ - спектральное множество $W$, а $N$ нулевое множество $I$. По предложению $2.3 \Lambda=N$. Пусть $\Lambda^{\prime}-$ квазиспектральное множество подпространства $W$. Ясно, что $\Lambda^{\prime} \subset N$. У тверждаем, что $\Lambda^{\prime}-$ квазинулевое множество $I$. Пусть $\varphi \in P$ и $\varphi \in I(\lambda) \forall \in \Lambda^{\prime}$. По свойству 8 дивизора включение $\varphi \in I(\lambda)$ эквивалентно включению $\operatorname{Div}_{\lambda} \varphi \supset \operatorname{Div}_{\lambda} I$. Таким образом, для $\lambda \in \Lambda^{\prime}$ и

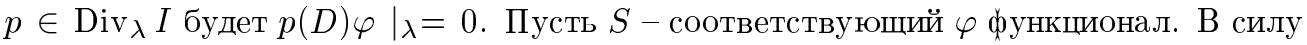
соотношения (5) функционал $S$ обрашается в нуль на всех $\exp -q$-многочленах из $W$ с показателями $\lambda \in \Lambda^{\prime}$. Поскольку по предположению замыкание линейной оболочки этих ехр- $q$-многочленов совпадает с $W, S$ аннулирует все $W$, т.е. $S \in W^{0}$. Это значит, что $\varphi \in I$.

Достаточность. Пусть $\Lambda^{\prime}$ - квазинулевое множество $I$. Из соотношения $\Lambda=N$ следует, что $\Lambda^{\prime}$ - часть спектрального множества $\Lambda$. Покажем, что $\Lambda^{\prime}-$ квазиспектральное множество. Для этого по теореме Хана-Банаха достаточно доказать следующее: если линейньй непрерывньй функционал $S$ обращается в нуль на элементарных 
exp-q-многочленах из $W$ с показателями $\lambda \in \Lambda^{\prime}$, то $S$ обращается в нуль на всем $W$. Пусть $S$ - такой функционал и $p(z) \exp \langle\lambda, z\rangle \in W, \lambda \in \Lambda^{\prime}$. Тогда $\langle S, p(z) \exp \langle\lambda, z\rangle\rangle=0$. Рассмотрим характеристический элемент $\varphi$ функционала $S$. Из соотношения (5) следует, что $\left.p(D) \varphi\right|_{\lambda}=0$. При фиксированном $\lambda \in \Lambda^{\prime}$ это верно для любого $p \in \operatorname{Sp}_{\lambda} W$. Поскольку $\operatorname{Sp}_{\lambda} W=\operatorname{Div}_{\lambda} I$, заключаем, что $\operatorname{Div}_{\lambda} \varphi \supset \operatorname{Div}_{\lambda} I, \lambda \in \Lambda^{\prime}$. В силу свойства 8 (п. 4) дивизора $\varphi \in I(\lambda)$. Это верно для любого $\lambda \in \Lambda^{\prime}$. Поскольку $\Lambda^{\prime}-$ квазинулевое множество $I$, заключаем, что $\varphi \in I$, или, что то же самое, $S \in W^{0}$. Иными словами, $S$ аннулирует $W$. Это и требовалось доказать.

Пусть $\Lambda$ - квазиспектральное множество инвариантного подпространства $W . K \boldsymbol{\text { в }} a$ зиспектром инвариантного подпространства мы называем дизъюнктное объединение

$$
\bigsqcup_{\lambda \in \Lambda} \operatorname{Sp}_{\lambda} W
$$

ПРЕДЛОЖЕНИЕ 2.5. Пусть инвариантные подпространства $W, W^{\prime}$ имеют одинаковие квазиспектры с квазиспектральным множеством $\Lambda$. Тогда $I(\lambda)=$ $I^{\prime}(\lambda) \forall \lambda \in \Lambda$.

ДоКАЗАТЕЛЬСТво. По условию

$$
\mathrm{Sp}_{\lambda} W=\operatorname{Sp}_{\lambda} W^{\prime} \forall \Lambda .
$$

По предложению 2.1

$$
\operatorname{Div}_{\lambda} I(\lambda)=\operatorname{Sp}_{\lambda} W=\operatorname{Sp}_{\lambda} W^{\prime}=\operatorname{Div}_{\lambda} I^{\prime}(\lambda)
$$

Таким образом, локальные дивизоры подмодулей $I$ и $I^{\prime}$ совпадают. По свойству 6 дивизоров (п. 4) совпадают и локальные подмодули.

ПреДЛОЖЕнИЕ 2.6. Пусть $\Omega \subset \Omega^{\prime}, a W, W^{\prime}$ допускают спектральный синтез с одним и тем же квазиспектром. Тогда $I \subset I^{\prime}$.

ДокАЗАТЕЛьство. Прежде всего отметим, что поскольку $W, W^{\prime}$ допускают спектральный синтез, их аннуляторные подмодули являются обильньми (п. 4). Далее, по условию $W$ и $W^{\prime}$ имеют один и тот же квазиспектр; следовательно, $W, W^{\prime}$ обладают одним и тем же спектральным множеством $\Lambda$ таким, что $\operatorname{Sp}_{\lambda} W=\operatorname{Sp}_{\lambda} W^{\prime}, \lambda \in \Lambda$. В силу предложения 2.5

$$
I(\lambda)=I^{\prime}(\lambda) \quad \forall \lambda \in \Lambda .
$$

Теперь приступим к доказательству включения $I \subset I^{\prime}$. Пусть $\varphi \in I$. Тогда $\varphi \in I(\lambda)$ $\forall \lambda \in \Lambda$. В силу (9), $\varphi \in I^{\prime}(\lambda) \forall \lambda \in \Lambda$. Кроме того, включение $\Omega \subset \Omega^{\prime}$ влечет включение $P \subset P^{\prime}$; следовательно, $\varphi \in P^{\prime}$. По предложению $2.4 \Lambda$ является квазинулевым множеством для $I^{\prime}$. По определению квазинулевого множества условие $\varphi \in P^{\prime}$ и условие $\varphi \in I^{\prime}(\lambda) \forall \lambda \in \Lambda$ влекут $\varphi \in I^{\prime}$. Включение $I \subset I^{\prime}$ установлено. 


\section{§ 3. Отображения инвариантных подпространств}

\section{1. Прямые и дуальные отображения.}

Пусть $H=H(\Omega), H^{\prime}=H\left(\Omega^{\prime}\right)$ - пространства, ассоциированные с открытыми $q$-множествами $\Omega, \Omega^{\prime} ; W, W^{\prime}$ - инвариантные подпространствав $H, H^{\prime}$ соответственно. Подпространства $W, W^{\prime}$ наделяются топологиями, индуцированными из пространств $H, H^{\prime}$, и рассматриваются как самостоятельные локально-вьпуклые пространства. Сопряженное $W^{*}$ к пространству $W$ изоморфно фактор-пространству $H^{*} / W^{0}$, наделенному фактор-топологией топологии $H^{*}$ по $W^{0}$ (см. [48; п. 9]); точно так же сопряженное $W^{\prime *}$ к пространству $W^{\prime}$ изоморфно фактор-пространству $H^{\prime *} / W^{\prime 0}$, наделенному фактор-топологией $H^{\prime *}$ по $W^{\prime 0}$. Пусть $P, P^{\prime}$ - модули, ассоциированные с $\Omega, \Omega^{\prime}$ соответственно; $I=\operatorname{An} W, I^{\prime}=\operatorname{An} W^{\prime}$. Очевидно, что $I, I^{\prime}$ - подмодули в $P, P^{\prime}$. Рассмотрим фактор-модули $P / I, P^{\prime} / I^{\prime}$. Наделим эти фактор-модули соответствуюшими фактор-топологиями. Изоморфизмы $T: H^{*} \rightarrow P, T^{\prime}: H^{\prime *} \rightarrow P^{\prime}$ порождают фактор-изоморфизмы

$$
\begin{gathered}
\widehat{T}: H^{*} / W^{0} \rightarrow P / I, \\
\widehat{T}^{\prime}: H^{\prime *} / W^{\prime 0} \rightarrow P^{\prime} / I^{\prime} .
\end{gathered}
$$

Пусть $u: W \rightarrow W^{\prime}$ - линейное непрерьвное отображение. Сопряженное отображение $u^{*}$ действует линейно и непрерывно из $H^{\prime *} / W^{\prime 0}$ в $H^{*} / W^{0}$. Отображения $\widehat{T}, \widehat{T}$ позволяют “поднять" отображение $u^{*}$ до отображения

$$
u^{\circledast}: P^{\prime} / I^{\prime} \rightarrow P / I
$$

именно,

$$
u^{\circledast}=\widehat{T} \circ u^{*} \circ \widehat{T}^{\prime-1} .
$$

Отображение $u^{\circledast}$ назьвается дуальным по отношению к прям⿻мм отображению $и$. Из соображений рефлексивности следует, что для всякого линейного непрерывного отображения $v: P^{\prime} / I^{\prime} \rightarrow P / I$ существует единственное прямое отображение $u: W \rightarrow W^{\prime}$, т.е. такое, что $v=u^{\circledast}$. Отметим, что если $u$ отображает $H$ в $H^{\prime}$, дуальное отображение $u^{\circledast}$ отображает $P^{\prime}$ в $P$. Известно, что $W, W^{\prime}$ - рефлексивные пространства Фреше. Поэтому для этих пространств справедливы известные утверждения Дьедонне-Шварца [48] о прямых и сопряженных операциях $u, u^{*}$; в этих утверждениях с соответствующей оговоркой можно заменить $u^{*}$ на $u^{\circledast}$.

2. Отображения, перестановочные с операторами частного дифференцирования.

Пусть $W, W^{\prime}$ - инвариантные подпространства в $H, H^{\prime}$ соответственно; $I, I^{\prime}$ - аннуляторные подмодули этих подпространств. Линейное отображение $u: W \rightarrow W^{\prime}$ перестановочно с операторами частного дифференцирования $D_{1}=\frac{\partial}{\partial z_{1}}, \ldots, D_{n}=\frac{\partial}{\partial z_{n}}$, если $D_{1} \circ u=u \circ D_{1}, \ldots, D_{n} \circ u=u \circ D_{n}$. Дуальная операция $D_{i}^{\circledast}$ к $D_{i}$ есть операция умножения на $i$-ю независимую переменную $\lambda_{i}$. Для перестановочности $u$ и $D_{i}$ необходимо и достаточно перестановочности $u^{\circledast}$ и $D^{\circledast}$, т.е.

$$
\lambda_{i} u^{\circledast}\left(\Phi^{\prime}\right)=u^{\circledast}\left(\lambda_{i} \Phi^{\prime}\right), \quad i=1, \ldots, n,
$$


где $\Phi^{\prime} \in P^{\prime} / I^{\prime}$. Стандартным образом соотношение (1) распространяется на многочлены:

$$
p u^{\circledast}\left(\Phi^{\prime}\right)=u^{\circledast}(p \Phi) \quad \forall p \in \mathbb{C}[\lambda] .
$$

Таким образом, справедливо

ПРЕДЛОЖЕНИЕ 3.1. Для того чтобы линейное непрерывное отображение $u: W \rightarrow W^{\prime}$ было перестановочно с операторами частного дифференцирования, необходимо и достаточно, чтобь дуальное отображсение $u^{\circledast}: P^{\prime} / I^{\prime} \rightarrow P / I$ было непрерывнылм модульныцм гомоморфизмом.

\section{3. Целые элементы.}

3.1. Элемент $f=\left(f_{1}, \ldots, f_{q}\right)$ из $H(\Omega)$ назьвается иельлм, если все компоненты $f_{i}$ допускают аналитическое продолжение до целых функций. Сдвигом $f_{h}$ целого элемента $f$ на шаг $h \in \mathbb{C}^{n}$ называется элемент

$$
f_{h}(z)=\left(f_{1}(z+h), \ldots, f_{q}(z+h)\right)
$$

Здесь мы используем запись элементов из $H(\Omega)$ в виде вектор-функций в соответствии с соглашением п. $1 \S 2$. Ясно, что если $f$ - цельй элемент, то и его сдвиг $f_{h}-$ целый элемент. Переход от $f$ к $f_{h}$ назьвается оператором сдвига и обозначается $T_{h}(f)$.

ПРЕДЛОЖЕНИЕ 3.2. Пусть $W$ - инвариантное подпространство в $H(\Omega)$ u $f-$ цельй әлемент из $W$. Тогда сдвиг $T_{h}(f)$ әлемента $f$ налюбой шаг $h \in \mathbb{C}^{n}$ снова принадлежстт $W$.

ДокаЗАТЕльство. Представим сдвиг $T_{h}(f)$ элемента $f$ в виде ряда Тейлора

$$
T_{h}(f)=\sum \frac{D^{\alpha}(f)}{\alpha !} h^{\alpha}
$$

Здесь $\alpha=\left(\alpha_{1}, \ldots, \alpha_{n}\right)$ - мультииндекс, $D^{\alpha}=D_{1}^{\alpha_{1}} \cdots D_{n}^{\alpha_{n}}, h^{\alpha}=h_{1}^{\alpha_{1}} \cdots h_{n}^{\alpha_{n}}$. С одной стороны, в силу инвариантности $W$ все производные $D^{\alpha} f$ принадлежат $W$ вместе c $f$. С другой стороны, ряд (2) при фиксированном $h$ сходится равномерно на каждом $q$-компакте в $\Omega$, т.е. в топологии $H(\Omega)$. В силу замкнутости $W$ сумма этого ряда, т.е. $T_{h}(f)$, принадлежит $W$.

3.2. Пусть теперь $W, W^{\prime}$ - инвариантные подпространства в $H(\Omega), H\left(\Omega^{\prime}\right) ; u: W \rightarrow$ $W^{\prime}$ - линейньй непрерьвньй оператор, перестановочньй с операторами частного дифференцирования. Если $f$ - целый элемент из $W$, то сдвиг $T_{h}(f)$ - снова целый элемент из $W$, и поэтому к нему можно применить оператор $u$, т.е. имеет смысл выражение $u \circ T_{h}(f)$.

ПРЕДЛОЖЕНИЕ 3.3. Для любого иелого әлемента $f \in W$ әлемент $u(f)$ является иельмм и справедливо соотношение

$$
u \circ T_{h}(f)=T_{h} \circ u(f) .
$$


ДокАЗАТЕЛЬСТВо. При фиксированном $h$ ряд (2) сходится равномерно в топологии $W$. Поэтому в силу непрерывности $u$ имеем

$$
u \circ T_{h}(f)=\sum \frac{u\left(D^{\alpha} f\right)}{\alpha !} h^{\alpha} .
$$

Пусть $F=u(f)$. В силу перестановочности оператора $u$ с операторами $D_{1}, \ldots, D_{n}$ справедливы равенства $u\left(D^{\alpha} f\right)=D^{\alpha} u(f)=D^{\alpha} F$. Таким образом,

$$
u \circ T_{h}(f)=\sum \frac{D^{\alpha} F}{\alpha !} h^{\alpha}=T_{h}(F)=T_{h} \circ u(f) .
$$

Соотношение (3) установлено.

По ходу доказательства установлено, что при любом фиксированном $h$ ряд

$$
\sum \frac{D^{\alpha} F}{\alpha !} h^{\alpha}
$$

сходится равномерно на каждом $q$-компакте в $\Omega$. Сумма этого ряда определяет аналитическое продолжение $F=u(f)$ до целого элемента.

\section{4. Преобразование элементарных ехр- $q$-многочленов.}

Пусть $u: W \rightarrow W^{\prime}$ - линейное непрерьвное отображение, перестановочное с операторами частного дифференцирования.

ПРЕДЛОЖЕНИЕ 3.4. Пусть $е$ - әлементарный ехр-q-многочлен в $W$ с показателем $\mu=\left(\mu_{1}, \ldots, \mu_{n}\right)$ и степенями $m_{1}, \ldots, m_{n}$ по переменныц $z_{1}, \ldots, z_{n}$ соответственно. Тогда образ $e^{\prime}=u(e)$ - элементарный ехр-q-многочлен с тем эке показателем $\mu$ и степенями, не превосходящими $m_{1}, \ldots, m_{n}$ соответственно по переменныц $z_{1}, \ldots, z_{n}$.

ДокАЗАТЕЛЬСТво. Используя перестановочность $D_{i}$ и $u$, имеем

$$
\left(D_{i}-\mu_{i} E\right)^{m_{i}+1} e^{\prime}=\left(D_{i}-\mu_{i} E\right)^{m_{i}+1} u(e)=u\left(\left(D_{i}-\mu_{i} E\right)^{m_{i}+1} e\right)=u(0)=0 .
$$

Здесь используется соотношение $\left(D_{i}-\mu_{i} E\right)^{m_{i}+1} e=0$, проистекающее из того, что степень ехр- $q$-многочлена $e$ по $z_{i}$ равна $m_{i}$. Это верно для любого $i=1, \ldots, n$. Таким образом, элемент $e^{\prime}$ удовлетворяет системе дифференциальных уравнений

$$
\left(D_{1}-\mu_{1} E\right)^{m_{1}+1} e^{\prime}=0, \ldots,\left(D_{n}-\mu_{n} E\right)^{m_{n}+1} e^{\prime}=0 .
$$

Согласно предложению $3.3 e^{\prime}$ - цельй элемент. Из теории обыкновенных дифференциальных уравнений известно, что всякое целое решение системы уравнений (4) представляет собою элементарный ехр- $q$-многочлен с показателем $\mu$ и степенями по переменным $z_{1}, \ldots, z_{n}$, не превосходящими $m_{1}, \ldots, m_{n}$ соответственно.

ПреДЛОЖенИЕ 3.5. Пусть $u: W \rightarrow W^{\prime}$ - линейное непрерывное отображение, $u^{\circledast}: P^{\prime} / I^{\prime} \rightarrow P / I-$ дуальное отображение, $е(z)=p(z) \exp \langle\lambda, z\rangle, e^{\prime}(z)=$ $p^{\prime}(z) \exp \langle\mu, z\rangle$ - әлементарные ехр-q-многочлены, содержащиеся соответственно в $W$ и $W^{\prime}$. Для того чтобы $e^{\prime}=u(e)$, необходимо и достаточно, чтобб для любого класса смезности $\Phi^{\prime} \in P^{\prime} / I^{\prime}$ и его образа $\Phi=u^{\circledast}\left(\Phi^{\prime}\right) \in P / I$ выполнялось соотношение

$$
\left.p(D) \Phi\right|_{\lambda}=\left.p^{\prime}(D) \Phi^{\prime}\right|_{\mu}
$$


ЗАмЕЧАНИЕ. Соотношение (5) следует понимать в следующем смысле: для любых $\varphi \in \Phi$ и $\varphi^{\prime} \in \Phi^{\prime}$ вьполняется соотношение

$$
\left.p(D) \varphi\right|_{\lambda}=\left.p^{\prime}(D) \varphi^{\prime}\right|_{\mu}
$$

ДокаЗАтельство. Необходимость. Пусть $e^{\prime}=u(e)$. Сопряженное отображение $u^{*}: H^{\prime *} / W^{\prime 0} \rightarrow H^{*} / W^{0}$ переводит класс смежности $S^{\prime} \in H^{\prime *} / W^{\prime 0}$, содержаший функционал $s^{\prime}$, в класс смежности $S \in H^{*} / W^{0}$, содержаший функционал $s$; при этом

$$
\langle s, f\rangle=\left\langle s^{\prime}, u(f)\right\rangle \quad \forall f \in W .
$$

Полагая в этом соотношении $f=e$, получим

$$
\langle s, e\rangle=\left\langle s^{\prime}, e^{\prime}\right\rangle
$$

или, что то же самое,

$$
\langle s, p(z) \exp \langle\lambda, z\rangle\rangle=\left\langle s^{\prime}, p^{\prime}(z) \exp \langle\mu, z\rangle\right\rangle .
$$

Это соотношение верно для любых функционалов $s \in S, s^{\prime} \in S^{\prime}$, где $S=u^{*}\left(S^{\prime}\right)$. Пусть $\varphi, \varphi^{\prime}$ - характеристические элементы $s, s^{\prime}$ соответственно. По определению дуального отображения $u^{\circledast}: P^{\prime} / I^{\prime} \rightarrow P / I$ оно переводит класс смежности $\Phi^{\prime} \in P^{\prime} / I^{\prime}$, содержащий элемент $\varphi^{\prime}$, в класс смежности $\Phi \in P / I$, содержащий элемент $\varphi$; при этом соответствуюшие функционалы $s$ и $s^{\prime}$ связаны соотношением (7). В силу формулы $(5) \S 2$ соотношение (7) можно записать в терминах характеристических элементов в виде $(6)$, где $\varphi \in \Phi, \varphi^{\prime} \in \Phi^{\prime}$ и $\Phi=u^{\circledast}\left(\Phi^{\prime}\right)$.

Достаточность. Пусть $\Phi=u^{\circledast}\left(\Phi^{\prime}\right)$ и выполняется соотношение (5). Это означает, что для любых представителей соответствующих классов вьполняется соотношение (6). В терминах соответствуюших функционалов $s, s^{\prime}$ соотношение (6) переписывается в виде

$$
\langle s, e\rangle=\left\langle s^{\prime}, e^{\prime}\right\rangle .
$$

Здесь $s$ - любой представитель класса смежности $S \in H^{*} / W^{0}, s^{\prime}$ - любой представитель класса $S^{\prime} \in H^{\prime *} / W^{\prime 0}$, причем $S=u^{*}\left(S^{\prime}\right)$. Соотношение (8) можно записать в эквивалентной форме

$$
\langle S, e\rangle=\left\langle S^{\prime}, e^{\prime}\right\rangle
$$

Отсюда следует

$$
\left\langle S^{\prime}, e^{\prime}\right\rangle=\left\langle u^{*}\left(S^{\prime}\right), e\right\rangle=\left\langle S^{\prime}, u(e)\right\rangle .
$$

Итак, $\left\langle S^{\prime}, e^{\prime}-u(e)\right\rangle=0$. Это верно для любого линейного непрерьвного функционала $S^{\prime}$ на $W^{\prime}$. По теореме Хана-Банаха $e^{\prime}-u(e)=0$.

5. Специальное свойство отображений, перестановочных с операторами дифференцирования.

Пусть $W, W^{\prime}$ - инвариантные подпространства в $H=H(\Omega), H^{\prime}=H\left(\Omega^{\prime}\right)$, причем $W$ допускает спектральньй синтез; $u: W \rightarrow W^{\prime}$ - линейное непрерывное отображение, перестановочное с операторами дифференцирования. Напомним, что для $q$-множества $\Omega$ и множества $A \subset \mathbb{C}^{n}$ символ $\Omega+A$ обозначает арифметическую сумму $\Omega$ и $A$ (см. $\S 2$, п. 1$)$. Обозначим $W_{A}$ (соответственно $\left.W_{A}^{\prime}\right)$ инвариантное подпространство в $H(\Omega+A)$ (соответственно в $\left.H\left(\Omega^{\prime}+A\right)\right)$, допускающее спектральньй синтез с тем же спектром, что и $W$ (соответственно $\left.W^{\prime}\right)$. 
ПреДЛОЖЕНИЕ 3.6. Для произвольного множества $A \subset \mathbb{C}^{n}$ существует линейное непрерьвное отображение $v: W_{A} \rightarrow W_{A}^{\prime}$ со свойствами:

1) v перестановочно с операторами частного дифференцирования;

2) для любого полинома Дирихле $е \in W$ вьполняется соотношение $v(e)=u(e)$.

ДокАЗАТЕЛЬСТво. Пусть $E$ - множество всех полиномов Дирихле из $W$. Очевидно, $E$ - линейное подпространство пространства $W_{A}$, плотное в нем. Наметим план рассуждений. Мы определим оператор $v_{0}: E \rightarrow W_{A}^{\prime}$; покажем, что этот оператор непрерывен в топологии, индуцируемой в $E$ из $W_{A}$; затем, пользуясь тем, что $E$ плотно в $W_{A}$, расширим $v_{0}$ до оператора $v$, действуюшего на всем $W_{A}$. Приступим к осуществлению этого плана.

Рассмотрим оператор $v_{0}$, который каждому полиному Дирихле $e \in E$ ставит в соответствие элемент $e^{\prime}=u(e)$. Согласно предложению $3.4, e^{\prime}-$ полином Дирихле. Поскольку $u$ отображает $E$ в $W^{\prime}, e^{\prime}$ принадлежит $W^{\prime}$; более того, $e^{\prime} \in W_{A}^{\prime}$, ибо $W_{A}^{\prime}$ содержит все полиномы Дирихле из $W^{\prime}$. Ясно, что $v_{0}$ линейно отображает $E$ в $W_{A}^{\prime}$. Отображение $v_{0}$ перестановочно с операторами частного дифференцирования. Действительно, используя инвариантность $E$ и перестановочность $u$ с операторами $D_{1}, \ldots, D_{n}$, имеем

$$
D_{i} \circ v_{0}(e)=D_{i} \circ u(e)=u \circ D_{i}(e)=v_{0} \circ D_{i}(e) .
$$

Наделим $E$ топологией, индуцированной из $H(\Omega+A)$, и покажем, что отображение $v_{0}: E \rightarrow W_{A}^{\prime}$ является непрерьвньм. Пусть $K-$ компакт в $\mathbb{C}^{n}$. Обозначим $H(K)$ банахово пространство функций, голоморфных внутри $K$ и непрерывных вплоть до гранищы $K$. Обозначим

$$
B(K)=\left\{f \in H(K): \max _{K}|f| \leqslant 1\right\}
$$

единичный шар в $H(K)$. Для $q$-компакта $\mathscr{K}=\left(K_{1}, \ldots, K_{q}\right)$ введем топологическое произведение $H(\mathscr{K}):=H\left(K_{1}\right) \times \cdots \times H\left(K_{q}\right)$. Это произведение есть банахово пространство с единичным шаром

$$
B(\mathscr{K})=B\left(K_{1}\right) \times \cdots \times B\left(K_{q}\right) .
$$

Семейства множеств

$$
\begin{aligned}
& \left\{\varepsilon B(\mathscr{K}) \cap W_{A}\right\}, \quad \mathscr{K} \Subset \Omega+A, \quad \varepsilon>0, \\
& \left\{\varepsilon B\left(\mathscr{K}^{\prime}\right) \cap W_{A}^{\prime}\right\}, \quad \mathscr{K}^{\prime} \Subset \Omega^{\prime}+A, \quad \varepsilon>0,
\end{aligned}
$$

образуют фундаментальные системы окрестностей нуля в $W_{A}$ и $W_{A}^{\prime}$ соответственно. Ввиду такого описания топологий рассматриваемых пространств достаточно доказать следующее утверждение: для каждого $q$-компакта $\mathscr{K}^{\prime} \Subset \Omega^{\prime}+A$ и каждого $\varepsilon>0$ сушествуют $q$-компакт $\mathscr{K} \subset \Omega+A$ и $\delta>0$ такие, что

$$
u(E \cap \delta B(\mathscr{K})) \subset \varepsilon B\left(\mathscr{K}^{\prime}\right) .
$$

Очевидно, что $q$-компакт $\mathscr{K}^{\prime}$ покрывается системой открытых $q$-множеств вида $\Omega^{\prime}+a, a \in A ;$ в свою очеред $\Omega^{\prime}$ покрывается системой открытых $q$-множеств $D^{\prime} \Subset \Omega^{\prime}$. 
Отсюда следует, что $q$-компакт $\mathscr{K}^{\prime}$ покрьвается конечным набором $q$-множеств вида $D_{j}^{\prime}+a_{j}, j=1, \ldots, N$, где $D_{j}^{\prime}-q$-компакты в $\Omega^{\prime}, a_{j} \in A$. Таким образом,

$$
\mathscr{K}^{\prime} \subset \bigcup_{j=1}^{N}\left(D_{j}^{\prime}+a_{j}\right) \text {. }
$$

Это влечет включение

$$
\bigcap_{j=1}^{N} B\left(D_{j}^{\prime}+a_{j}\right) \subset B\left(\mathscr{K}^{\prime}\right) .
$$

В силу непрерывности отображения $u: W \rightarrow W^{\prime}$ сушествуют $q$-компакты $D_{j} \subset \Omega$, $j=1, \ldots, N$, и $\delta>0$ такие, что

$$
u\left(\delta B\left(D_{j}\right) \cap W\right) \subset \varepsilon B\left(D_{j}^{\prime}\right) .
$$

Полагаем

$$
\mathscr{K}=\bigcup_{j=1}^{N}\left(D_{j}+a_{j}\right) .
$$

Пусть $e \in E$ и $e^{\prime}=u(e)$. Докажем импликацию

$$
e \in \delta B(\mathscr{K}) \Longrightarrow e^{\prime} \in \varepsilon B\left(\mathscr{K}^{\prime}\right) .
$$

Включение $e \in \delta B(\mathscr{K})$ равносильно $N$ включениям

$$
e \in \delta B\left(D_{j}+a_{j}\right), \quad j=1, \ldots, N .
$$

Рассмотрим сдвиги $e_{j}=T_{a_{j}}(e)$. Включения (11) равносильны включениям

$$
e_{j} \in \delta B\left(D_{j}\right), \quad j=1, \ldots, N .
$$

Положим $e_{j}^{\prime}=u\left(e_{j}\right), j=1, \ldots, N$. Включения (12) и (9) влекут

$$
e_{j}^{\prime} \in \varepsilon B\left(D_{j}^{\prime}\right), \quad j=1, \ldots, N .
$$

Но согласно предложению 3.3

$$
e_{j}^{\prime}=u\left(T_{a_{j}}(e)\right)=T_{a_{j}}(u(e))=T_{a_{j}}\left(e^{\prime}\right) .
$$

Из вьшеизложенного следует, что

$$
T_{a_{j}}\left(e^{\prime}\right) \in \varepsilon B\left(D_{j}^{\prime}\right), \quad j=1, \ldots, N .
$$

Эти включения можем записать в равносильной форме

$$
e^{\prime} \in \varepsilon B\left(D_{j}^{\prime}+a_{j}\right), \quad j=1, \ldots, N .
$$

Это означает, что

$$
e^{\prime} \in \varepsilon \bigcap_{j=1}^{N} B\left(D_{j}^{\prime}+a_{j}\right) \subset \varepsilon B\left(\mathscr{K}^{\prime}\right) .
$$

Импликация (10) доказана. Тем самым установлено, что отображение $v_{0}$ действует непрерьвно из $E$ в $W_{A}^{\prime}$. Поскольку $E$ плотно в $W_{A}$, а $W_{A}^{\prime}$ полно, отображение $v_{0}$ по непрерьвности распространяется на все $W_{A}$. Это расширение $v$ будет линейным. В силу бочечности $W_{A}[45 ;$ гл. XI] оно будет непрерывным; при этом образ расширенного отображения будет по-прежнему лежать в $W_{A}^{\prime}$. Построенное отображение $v: W_{A} \rightarrow W_{A}^{\prime}$ удовлетворяет всем требованиям: оно непрерьвно; перестановочно с операторами частного дифференцирования, ибо таким свойством обладает его сужение $v_{0}$ на подпространство $E$, плотное в $W_{A}$; в силу построения вьполняется соотношение $v(e)=u(e)$ для любого $e \in E$. 


\section{$\S$ 4. $\Theta$-выпуклость}

1. Определение.

Пространство $\mathbb{C}^{n} n$-мерных комплексных векторов мы отождествляем с евклидовым пространством $\mathbb{R}^{2 n}$ вешественных $2 n$-мерных векторов с помошью изоморфизма

$$
\alpha: z=\left(z_{1}, \ldots, z_{n}\right) \rightarrow w=\left(x_{1}, y_{1}, \ldots, x_{n}, y_{n}\right)
$$

где $x_{i}=\operatorname{Re} z_{i}, y_{i}=\operatorname{Im} z_{i}$. Скалярное произведение $\left(z^{\prime}, z^{\prime \prime}\right)$ в $\mathbb{C}^{n}$ вводится по правилу

$$
\left(z^{\prime}, z^{\prime \prime}\right)=\operatorname{Re} \sum_{j=1}^{n} z_{j}^{\prime} \bar{z}_{j}^{\prime \prime}=\operatorname{Re} \sum_{j=1}^{n}\left(x_{j}^{\prime}+i y_{j}^{\prime}\right)\left(x_{j}^{\prime \prime}-i y_{j}^{\prime \prime}\right)=\sum_{j=1}^{n}\left(x_{j}^{\prime} x_{j}^{\prime \prime}+y_{j}^{\prime} y_{j}^{\prime \prime}\right)=\left(w^{\prime}, w^{\prime \prime}\right) \text {. }
$$

Вместе со скалярным произведением в $\mathbb{C}^{n}$ вводятся геометрические понятия расстояния, ортогональности, вьпуклости, вьпуклой оболочки и т. д. Расстояние $|z|$ точки $z$ от начала координат вычисляется по формуле

$$
|z|^{2}=(z, z)=\sum_{j=1}^{n}\left(x_{j}^{2}+y_{j}^{2}\right)=|w|^{2}
$$

Пусть $S^{2 n-1}=\left\{\tau \in \mathbb{C}^{n}:|\tau|=1\right\}$ - единичная сфера в $\mathbb{C}^{n}$. Каждый элемент $\tau$ единичной сферы задает луч $t \tau, t>0$, уходящий в бесконечность в направлении $\tau$. Поэтому элементы единичной сферы удобно назьвать направлениями. $\tau$-гиперплоскость в $\mathbb{C}^{n}$, находяшаяся на "расстоянии" $p$ от начала ("расстояние" снабжено знаком), задается уравнением

$$
(z, \tau)=p,
$$

где $p \in \mathbb{R}^{n}$. Эта гиперплоскость является общей границей двух полупространств: замкнутого полупространства

$$
\Pi(\tau, p)=\left\{z \in \mathbb{C}^{n}:(z, \tau) \leqslant p\right\}
$$

и открытого полупространства

$$
\Pi^{0}(\tau, p)=\left\{z \in \mathbb{C}^{n}:(z, \tau)<p\right\} .
$$

Полупространства вида (1), (2) будем назьвать $\tau$-полупространствами. Условимся, что при $p=-\infty$ любое $\tau$-полупространство пусто, а при $p=\infty$ совпадает с $\mathbb{C}^{n}$.

Напомним, что множество $M$ в $\mathbb{C}^{n}$ назьвается вылукльљм, если вместе с двумя точками $z_{1}, z_{2}$ оно содержит отрезок $\left[z_{1}, z_{2}\right]$. Вьпуклое замкнутое множество представляется как пересечение замкнутых полупространств $[49 ; \S 11$, теорема 11.5$]$; открытое вьпуклое множество - как пересечение открытых полупространств [49; $\S 11$, теоремa 11.3].

Пусть $\Theta$ - некоторое множество направлений на сфере $S^{2 n-1}$. Множество $L \subset \mathbb{C}^{n}$ называется $\Theta$-вылуклым, если оно представляется как пересечение некоторого семейства $\tau$-полупространств, $\tau \in \Theta$. Если $\Theta^{\prime} \subset \Theta^{\prime \prime}$, то согласно определению всякое $\Theta^{\prime}$-выпуклое множество является и $\Theta^{\prime \prime}$-выпуклым. Пересечение любого семейства $\Theta$-вьпуклых множеств является $\Theta$-вьпуклым. Поэтому для любого множества 
$L \subset \mathbb{C}^{n}$ существует наименьшее $\Theta$-выпуклое множество, его содержащее, - пересечение всех $\Theta$-выпуклых множеств, содержащих $L$. Это множество назьвается $\Theta$-в впуклой оболочкой множества $L$ и обозначается $\operatorname{conv}_{\Theta} L$. Очевидно, что conve $L \subset$ $\operatorname{conv}_{\Theta} L^{\prime}$ при $L \subset L^{\prime}$. Далее, $\Theta$-вьпуклую оболочку открытого множества можно представить как пересечение всех открытых $\tau$-полупространств, его содержаших, а $\Theta$-вьпуклую оболочку компакта - как пересечение всех замкнутых полупространств, его содержаших $(\tau \in \Theta)$. Из последнего утверждения, в частности, следует, что $\Theta-$-выпуклая оболочка компакта - замкнутое множество. Отметим, что если $\Theta=S^{2 n-1}$, а $L$ - либо компакт, либо открытое множество, то $\Theta$-выпуклая оболочка множества $L$ совпадает с его выпуклой оболочкой. Насколько нам известно, понятие плоского $\Theta$-выпуклого множества было впервые введено в 1973 г. в работе [35]. Замкнутые $\Theta$-вьпуклые множества в $\mathbb{R}^{n}$ (под названием $H$-выпуклых) были введены В. Г. Болтянским (см. [50], [51]) в 1976 г. в связи с теоремой Хелли [52]. Свойства замкнутых $\Theta$-вьпуклых множеств исследованы в книгах [53], [54].

\section{2. Элементарные свойства.}

ПРЕДЛОЖЕНИЕ 4.1. Пусть $\Theta$ - замкнутое множсество направлений, $G$ - открытое множество. Тогда $\Theta$-выпуклая оболочка множества $G$ - открытое множество.

ДокаЗАТЕльство. Пусть $z_{0} \in \operatorname{conv}_{\Theta} G$. Нужно показать, что $z_{0}$ - внутренняя точка $\operatorname{conv}_{\Theta} G$, т.е. существует шар положительного радиуса с центром в $z_{0}$, содержащийся в $\operatorname{conv}_{\Theta} G$. Для этого достаточно показать, что существует положительное число $\delta>0$ такое, что расстояние $d(\tau, p)$ точки $z_{0}$ до границы открытого полупространства $\Pi^{0}(\tau, p), \tau \in \Theta$, содержашего $G$, не менее $\delta$. Предположим противное, т.е. сушествует последовательность открытых полупространств $\Pi^{0}\left(\tau_{n}, p_{n}\right), \tau_{n} \in \Theta$, содержаших $G$, такая, что $d\left(\tau_{n}, p_{n}\right) \rightarrow 0$. Заметим, что $d\left(\tau_{n}, p_{n}\right)=p_{n}-\left(z_{0}, \tau_{n}\right)$. Таким образом, согласно предположению

$$
p_{n}-\left(z_{0}, \tau_{n}\right) \rightarrow 0
$$

Последовательность чисел $\left(z_{0}, \tau_{n}\right)$ ограничена, так как $\left|\left(z_{0}, \tau_{n}\right)\right| \leqslant\left|z_{0}\right|$.

Из (3) следует, что и последовательность $p_{n}, n=1,2, \ldots$, ограничена. Поэтому сушествует подпоследовательность $\mathbb{N}^{\prime}$ натурального ряда такая, что $p_{n} \rightarrow p_{0}, \tau_{n} \rightarrow \tau_{0}$ при $n \rightarrow \infty, n \in \mathbb{N}^{\prime}$. В силу замкнутости $\Theta$ будет $\tau_{0} \in \Theta$. Совершая в $(3)$ предельньй переход, получим

$$
\left(z_{0}, \tau_{0}\right)=p_{0}
$$

Далее, так как $G \subset \Pi^{0}\left(\tau_{n}, p_{n}\right)$, то для любой точки $z \in G$ имеем

$$
\left(z, \tau_{n}\right)<p_{n}, \quad n=1,2, \ldots
$$

Совершая предельный переход, получим

$$
\left(z, \tau_{0}\right) \leqslant p_{0}
$$

Это неравенство справедливо для любой точки $z \in G$. Поскольку образ $G$ при отображении $z \rightarrow\left(z, \tau_{0}\right)$ открыт, в действительности будет вьполняться строгое неравенство

$$
\left(z, \tau_{0}\right)<p_{0} \quad \forall z \in G .
$$


Это неравенство означает, что открытое полупространство $\Pi^{0}\left(\tau_{0}, p_{0}\right)$ содержит $G$. $\mathrm{C}$ другой стороны, равенство (4) означает, что точка $z_{0}$ принадлежит границе открытого $\tau_{0}$-полупространства $\Pi^{0}\left(\tau_{0}, p_{0}\right), \tau_{0} \in \Theta$, содержащего $G$, и, значит, не может принадлежать $G$. Это противоречие заставляет признать, что существует положительное $\delta$ такое, что $d(\tau, p) \geqslant \delta$ для всякого полупространства $\Pi^{0}(\tau, p)$, содержашего $G$. Тем самьм, предложение доказано.

ПрЕДЛОЖЕНИЕ 4.2. Пусть $\Omega$ - ограниченное открытое множество, $\bar{\Omega}-$ замыкание $\Omega$. Тогда

$$
\overline{\operatorname{conv}_{\Theta} \Omega}=\operatorname{conv}_{\Theta} \bar{\Omega} .
$$

ДоКАЗАТЕЛЬСТВО. Поскольку $\Omega \subset \bar{\Omega}$,

$$
\operatorname{conv}_{\Theta} \Omega \subset \operatorname{conv}_{\Theta} \bar{\Omega} \text {. }
$$

В силу ограниченности $\Omega$ множество $\bar{\Omega}$ является компактом, и, следовательно, conv $\Theta \bar{\Omega}$ замкнуто (см. п. 1). После операции замыкания получим

$$
\overline{\operatorname{conv}_{\Theta} \Omega} \subset \operatorname{conv}_{\Theta} \bar{\Omega} .
$$

Теперь будем доказьвать обратное включение. Множества $\Omega$ и $\bar{\Omega}$ имеют одну и ту же опорную функцию (в смысле вещественного анализа)

$$
H_{\Omega}^{(r)}(z)=H_{\bar{\Omega}}^{(r)}(z)=\sup _{\zeta \in \Omega}(\zeta, z) .
$$

Множество conv $_{\Theta} \bar{\Omega}$ представляется как пересечение всех замкнутых $\tau$-полупространств, $\tau \in \Theta$, содержаших $\Omega$, а $\operatorname{conv}_{\Theta} \Omega$ - как пересечение всех открытых $\tau$-полупространств, $\tau \in \Theta$, содержащих $\Omega$. Это позволяет представить $\Theta$-вьпуклые оболочки множеств $\bar{\Omega}$ и $\Omega$ в виде

$$
\begin{aligned}
& \operatorname{conv}_{\Theta} \bar{\Omega}=\left\{z:(z, \tau) \leqslant H^{(r)}(\tau) \forall \tau \in \Theta\right\}, \\
& \operatorname{conv}_{\Theta} \Omega=\left\{z:(z, \tau)<H^{(r)}(\tau) \forall \tau \in \Theta\right\} .
\end{aligned}
$$

Пусть $z_{0} \in \operatorname{conv}_{\Theta} \bar{\Omega}$. Согласно (7) это значит, что

$$
\left(z_{0}, \tau\right) \leqslant H^{(r)}(\tau) \quad \forall \tau \in \Theta .
$$

Фиксируем точку $z_{0}^{\prime} \in \Omega$, отличную от $z_{0}$. Согласно (8) имеем

$$
\left(z_{0}^{\prime}, \tau\right)<H^{(r)}(\tau) \quad \forall \tau \in \Theta .
$$

Рассмотрим интервал $\left(z_{0}^{\prime}, z_{0}\right)$. Этот интервал не пуст, так как $z_{0}^{\prime} \neq z_{0}$. Утверждаем, что каждая точка этого интервала принадлежит $\operatorname{conv}_{\Theta} \Omega$. Пусть $z \in\left(z_{0}^{\prime}, z_{0}\right)$. Тогда $z$ представляется в виде $z=\alpha z_{0}^{\prime}+\beta z_{0}$, где $\alpha>0, \beta>0, \alpha+\beta=1$. Отсюда для любого направления $\tau \in \Theta$ имеем $(z, \tau)=\alpha\left(z_{0}^{\prime}, \tau\right)+\beta\left(z_{0}, \tau\right)$. Поскольку $\alpha>0$ и для точек $z_{0}^{\prime}$, $z_{0}$ выполняются неравенства $(9),(10)$, получаем неравенство

$$
(z, \tau)<H^{(r)}(\tau) .
$$

Это верно для любого направления $\tau \in \Theta$. Согласно (8) заключаем, что $z \in \operatorname{conv}_{\Theta} \Omega$. Таким образом, мы показали, что $\left(z_{0}^{\prime}, z_{0}\right) \subset \operatorname{conv}_{\Theta} \Omega$. Но любая окрестность $z_{0}$ пересекается с $\left(z_{0}^{\prime}, z_{0}\right)$. Значит, $z_{0}$ есть точка прикосновения для $\operatorname{conv}_{\Theta} \Omega$, т.е. $z_{0} \in$ $\overline{\operatorname{conv}_{\Theta} \Omega}$. Это верно для любой точки $z_{0} \in \operatorname{conv}_{\Theta} \bar{\Omega}$. Значит, имеет место включение

$$
\operatorname{conv}_{\Theta} \bar{\Omega} \subset \overline{\operatorname{conv}_{\Theta} \Omega} .
$$

Тем самьм, соотношение (5) установлено. 
ПРЕДЛОЖЕНИЕ 4.3. Пусть $\Theta$ - множество направлений, $\bar{\Theta}-$ замыкание $\Theta$. Тогда для любого компакта D справедливо соотношение

$$
\operatorname{conv}_{\Theta} D=\operatorname{conv}_{\Theta} D
$$

ДокАЗАтельство. Так как $\Theta \subset \bar{\Theta}$, то семейство $\tau$-полупространств, $\tau \in \bar{\Theta}$, содержаших $D$, шире семейства $\tau$-полупространств, $\tau \in \Theta$, содержащих $D$. Поэтому

$$
\operatorname{conv}_{\Theta} D \subset \operatorname{conv}_{\Theta} D
$$

Докажем обратное включение. Пусть $x \notin \operatorname{conv}_{\Theta} D$. Тогда сушествует замкнутое $\tau_{0}$-полупространство $\Pi\left(\tau_{0}, p\right), \tau_{0} \in \bar{\Theta}$, такое, что $D \subset \Pi\left(\tau_{0}, p\right)$, но $x \notin \Pi\left(\tau_{0}, p\right)$. Путем малого “шевеления" $p$ можно добиться того, что $D \subset \operatorname{Int} \Pi\left(\tau_{0}, p\right)$, но по-прежнему $x \notin \Pi\left(\tau_{0}, p\right)$. Далее, существует последовательность направлений $\tau_{n} \rightarrow \tau_{0}, \tau_{n} \in \Theta$. Последовательность полупространств П $\left(\tau_{n}, p\right)$ будет сходиться к полупространству $\Pi\left(\tau_{0}, p\right)$, и в силу того, что $D$ компактно принадлежит $\Pi\left(\tau_{0}, p\right)$, найдется достаточно большое $n$ такое, что П $\left(\tau_{n}, p\right)$ содержит $D$, но не содержит $x$. Это означает, что $x \notin$ $\operatorname{conv}_{\Theta} D$. Таким образом, справедлива импликация

$$
x \notin \operatorname{conv}_{\Theta} D \Longrightarrow x \notin \operatorname{conv}_{\Theta} D
$$

Эта импликация равносильна тому, что

$$
\operatorname{conv}_{\Theta} D \subset \operatorname{conv}_{\Theta} D
$$

Предложение доказано.

Интересно отметить, что аналогичное утверждение для открытых множеств не верно. В качестве примера рассмотрим открытьй шар $B$ единичного радиуса с центром в начале координат. В качестве $\Theta$ возьмем $S^{2 n-1} \backslash \tau_{0}$, где $\tau_{0}$ - точка $S^{2 n-1}$. Замыкание $\bar{\Theta}$ множества $\Theta$ совпадает с $S^{2 n-1}$. Однако $\operatorname{conv}_{\Theta} B=B \cup\left\{\tau_{0}\right\}$, a $\operatorname{conv}_{\bar{\Theta}} B=$ $\operatorname{conv}_{S^{2 n-1}} B=B$.

\section{3. Предельный переход.}

ПРЕДЛОЖЕНИЕ 4.4. Пусть $G$ - открытое множество, $\Theta$ - замкнутое множество направлений, $K_{1} \Subset K_{2} \Subset \cdots-$ возрастающая последовательность компактов, исчерпьвающая $G$. Тогда

$$
\operatorname{conv}_{\Theta} G=\bigcup_{n=1}^{\infty} \operatorname{conv}_{\Theta} K_{n}
$$


ДокАЗАТЕЛЬСтво. Так как $K_{n} \subset G$, то

$$
\operatorname{conv}_{\Theta} K_{n} \subset \operatorname{conv}_{\Theta} G
$$

и потому

$$
\bigcup_{n=1}^{\infty} \operatorname{conv}_{\Theta} K_{n} \subset \operatorname{conv}_{\Theta} G
$$

Докажем обратное включение. Если $\bigcup_{n=1}^{\infty} \operatorname{conv}_{\Theta} K_{n}=\mathbb{C}^{n}$, то из (12) следует (11) и предложение доказано. Поэтому предположим, что $\bigcup_{n=1}^{\infty} \operatorname{conv}_{\Theta} K_{n} \neq \mathbb{C}^{n}$. Пусть $z^{0} \notin \bigcup_{n=1}^{\infty} \operatorname{conv}_{\Theta} K_{n}$. Тогда $z^{0} \notin \operatorname{conv}_{\Theta} K_{n}$ при любом $n=1,2, \ldots$ Это значит, что для любого $n$ существует замкнутое полупространство $\Pi\left(\tau_{n}, p_{n}\right), \tau_{n} \in \Theta$, содержащее $K_{n}$, но не содержашее $z^{0}$, т.е.

$$
\begin{gathered}
\left(z, \tau_{n}\right) \leqslant p_{n}, \quad \forall z \in K_{n}, \\
\left(z^{0}, \tau_{n}\right)>p_{n} .
\end{gathered}
$$

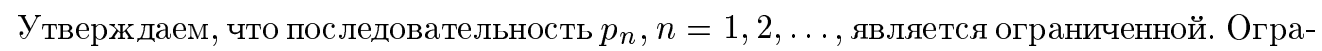
ниченность сверху следует из неравенства

$$
p_{n}<\left(z_{0}, \tau_{n}\right) \leqslant\left|z^{0}\right| .
$$

Ограниченность снизу следует из следующего соображения: фиксируем точку $z_{0} \in G$; при достаточно больших $n$ (скажем, $\left.n>n_{0}\right) z_{0} \in K_{n}$, тем более, $z_{0} \in \Pi\left(\tau_{n}, p_{n}\right)$, и, значит, $\left(z_{0}, \tau_{n}\right) \leqslant p_{n}$; отсюда следует, что $p_{n} \geqslant-\left|\left(z_{0}, \tau_{n}\right)\right| \geqslant-\left|z_{0}\right|$. Пользуясь тем, что $\Theta$ замкнуто и, следовательно, компактно на сфере $S^{2 n-1}$, а последовательность $p_{n}$ ограниченна, выделим подпоследовательность $\mathbb{N}^{\prime}$ натурального ряда такую, что при $n \rightarrow \infty, n \in \mathbb{N}^{\prime}$, будет $\tau_{n} \rightarrow \tau_{0}, \tau_{0} \in \Theta$, и $p_{n} \rightarrow p_{0}$. Совершая предельный переход в соотношениях (13), (14), получим

$$
\begin{gathered}
\left(z, \tau_{0}\right) \leqslant p_{0} \quad \forall z \in G, \\
\left(z^{0}, \tau_{0}\right) \geqslant p_{0} .
\end{gathered}
$$

Неравенство (15) справедливо при любом $z \in G$. Поскольку образ $G$ при отображении $z \rightarrow\left(z, \tau_{0}\right)$ открыт, в действительности для каждого $z \in G$ будет вьполняться неравенство

$$
\left(z, \tau_{0}\right)<p .
$$

Неравенства (16) и (17) равносильны тому, что открытое полупространство $\Pi^{0}\left(\tau_{0}, p_{0}\right)$ содержит $G$, но не содержит $z^{0}$. С другой стороны, как подчеркивалось выше (п. 1), conv $_{\Theta} G$ представляется в виде пересечения всех открытых $\tau$-полупространств, $\tau \in \Theta$, содержаших $G$. Следовательно, $z^{0} \notin \operatorname{conv}_{\Theta} G$. Таким образом,

$$
z \notin \bigcup_{n=1}^{\infty} \operatorname{conv}_{\Theta} K_{n} \Longrightarrow z \notin \operatorname{conv}_{\Theta} G
$$

Это означает, что $\operatorname{conv}_{\Theta} G \subset \bigcup_{n=1}^{\infty} \operatorname{conv}_{\Theta} K_{n}$. Тем самьм, соотношение (11) установлено. 
Отметим, что в завуалированной форме доказанное утверждение содержится в статье К. Кисельмана [38; предложение 3.2].

\section{4. Об опорных функциях компактов.}

Для неограниченного множества $Z \subset \mathbb{C}^{n}$ обозначим $\Theta_{Z}$ множество предельных направлений этого множества, т.е. множество точек $\tau$ на единичной сфере $S^{2 n-1} \subset \mathbb{C}^{n}$, для которых существуют последовательности точек $z_{n} \in Z$ таких, что $z_{n} /\left|z_{n}\right| \rightarrow \tau$. Множество предельных направлений любого неограниченного множества $Z$ является замкнутым. Если $Z$ ограничено, то полагаем $\Theta_{Z}=\varnothing$.

Пусть $K-$ компакт в $\mathbb{C}^{n}$. Функция

$$
H^{(r)}(\lambda)=\sup _{z \in \mathbb{C}^{n}}(z, \lambda)=\sup _{z \in K} \operatorname{Re}(z, \bar{\lambda})
$$

называется опорной функиией компакта $K$ в смысле вещественного анализа. Эта функция связана с соответствующей опорной функцией в смысле комплексного анализа $(\S 1$, п. 4$)$ соотношением

$$
H_{K}^{(r)}(\lambda)=H_{K}(\bar{\lambda})
$$

где $\lambda=\left(\bar{\lambda}_{1}, \ldots, \bar{\lambda}_{n}\right)$ - вектор, комплексно сопряженньй $\lambda=\left(\lambda_{1}, \ldots, \lambda_{n}\right)$.

ПРЕДЛОЖЕНИЕ 4.5. Пусть $G$ - открытое множество, $M$ - неограниченное множсество в $\mathbb{C}^{n}, \Theta-$ множество предельных направлений этого множества. Тогда для любого компакта $K^{\prime} \subset \operatorname{conv}_{\Theta} G$ существуют компакт $K \subset G u$ константы $\varepsilon>0, c<\infty$ такие, что

$$
H_{K^{\prime}}^{(r)}(z) \leqslant H_{K}^{(r)}(z)-\varepsilon|z|+c
$$

nрu $z \in M$.

ДоКАЗАтЕЛЬСтво. Рассмотрим возрастающую последовательность компактов

$$
D_{1} \Subset D_{2} \Subset \cdots,
$$

исчерпывающую $G$. Согласно предложению 4.4

$$
\operatorname{conv}_{\Theta} G=\bigcup_{n=1}^{\infty} \operatorname{conv}_{\Theta} D_{n}
$$

По условию $D_{n-1} \subset \operatorname{Int} D_{n}$, следовательно,

$$
\operatorname{conv}_{\Theta} D_{n-1} \subset \operatorname{conv}_{\Theta} \operatorname{Int} D_{n} \subset \operatorname{conv}_{\Theta} D_{n}
$$

В силу этих включений и (19) $\operatorname{conv}_{\Theta} G$ представляется в виде объединения последовательности множеств conve Int $D_{n}$; при этом по предложению 4.1 множества conve Int $D_{n}$ открыты. Фиксируем компакт $K^{\prime} \subset \operatorname{conv}_{\Theta} G$. Возрастающая последовательность открытых множеств conv $\operatorname{Int}_{n}$ покрывает $K^{\prime}$. Поэтому найдется номер $n_{0}$ такой, что $K^{\prime} \subset \operatorname{conv}_{\Theta} \operatorname{Int} D_{n_{0}}$. Из этого включения следует, что 
$H_{K^{\prime}}^{(r)}(\tau) \leqslant H_{D_{n_{0}}}^{(r)}(\tau), \tau \in \Theta$. Далее, поскольку $D_{n_{0}} \Subset D_{n_{0}+1}$, найдется $\varepsilon>0$ такое, что

$$
H_{D_{n_{0}}}^{(r)}(\tau) \leqslant H_{D_{n_{0}+1}}^{(r)}(\tau)-2 \varepsilon .
$$

Таким образом,

$$
H_{K^{\prime}}^{(r)}(\tau) \leqslant H_{D_{n_{0}+1}}^{(r)}(\tau)-2 \varepsilon, \quad \tau \in \Theta .
$$

Положим $K=D_{n_{0}+1}$ и покажем, что при таком выборе $K$ будет иметь место (18). Запишем неравенство (20) в виде

$$
H_{K^{\prime}}^{(r)}\left(\tau^{\prime}\right) \leqslant H_{K}^{(r)}\left(\tau^{\prime}\right)-2 \varepsilon, \quad \tau^{\prime} \in \Theta .
$$

Пусть $z=\tau|z| \in M$, где $\tau$ - точка на единичной сфере. Сопоставим точке $\tau$ одну из ближайших к ней точек $\tau^{\prime} \in \Theta$. Это можно сделать, так как $\Theta$ - компакт на единичной сфере. Используя неравенство (21), имеем

$$
\begin{aligned}
H_{K^{\prime}}^{(r)}(z) & =|z| H_{K^{\prime}}^{(r)}(\tau)=|z|\left(H_{K^{\prime}}^{(r)}(\tau)-H_{K^{\prime}}^{(r)}\left(\tau^{\prime}\right)\right)+|z| H_{K^{\prime}}^{(r)}\left(\tau^{\prime}\right) \\
& \leqslant|z|\left(H_{K^{\prime}}^{(r)}(\tau)-H_{K^{\prime}}^{(r)}\left(\tau^{\prime}\right)\right)+|z|\left(H_{K}^{(r)}\left(\tau^{\prime}\right)-H_{K}^{(r)}(\tau)\right)+|z| H_{K}^{(r)}(\tau)-2 \varepsilon|z| .
\end{aligned}
$$

При $z \rightarrow \infty, z \in M$, справедливо соотношение $\tau-\tau^{\prime}=o(1)$ и, следовательно, в силу равномерной непрерьвности $H_{K}^{(r)}$ и $H_{K^{\prime}}^{(r)}$ на единичной сфере $H_{K^{\prime}}^{(r)}(\tau)-H_{K^{\prime}}^{(r)}\left(\tau^{\prime}\right)=$ $o(1), H_{K}^{(r)}\left(\tau^{\prime}\right)-H_{K}^{(r)}(\tau)=o(1)$. Таким образом, при $z \rightarrow \infty, z \in M$, и достаточно большом $C$ имеем

$$
H_{K^{\prime}}^{(r)}(z) \leqslant o(|z|)-2 \varepsilon|z|+|z| H_{K}^{(r)}(\tau) \leqslant c+\varepsilon|z|+H_{K}^{(r)}(z) .
$$

Предложение доказано.

\section{$\S$ 5. Нестандартная выпуклость}

Возможность аналитического продолжения элементов из инвариантных подпространств связана со специальным свойством $(A, B)$-вьпуклости $q$-областей существования этих элементов. Это свойство является частным случаем более общей выпуклости, описанной в книге Л. Данцера, Б. Грюнбаума и В. Кли [52; §9].

1. Геометрическая разность $q$-множеств.

Пусть $K=\left(K_{1}, \ldots, K_{q}\right)-q$-множество, $A \subset \mathbb{C}^{n}$. Напомним, что $q$-множество $K+A:=\left(K_{1}+A, \ldots, K_{q}+A\right)$ назьвается арифметической суммой $q$-множества $K$ и множества $A \subset \mathbb{C}^{n}$. В частности, если $a \in \mathbb{C}^{n}$, то $K+a:=\left(K_{1}+a, \ldots, K_{q}+a\right)-$ сдвиг $q$-множества на вектор $a$. Пусть $K, K^{\prime}-q$-множества. Множество $K^{\prime} \ominus K:=$ $\left\{a \in \mathbb{C}^{n}: K+a \subset K^{\prime}\right\}$ называется геометрической разностью множеств $K^{\prime}$ и $K$. Очевидно, $K^{\prime} \ominus K \subset \mathbb{C}^{n}$ и $K+\left(K^{\prime} \ominus K\right) \subset K^{\prime}$.

В том случае, когда никакой сдвиг $K+a$ не содержится в $K^{\prime}$, геометрическая разность $K^{\prime} \ominus K$ представляет собою пустое множество. Нам понадобятся следуюшие свойства геометрической разности.

Свойство 1. Пусть $\Omega$ - открытое q-множество, $A$-q-компакт. Тогда $\Omega \ominus A$ - открытое множество.

Доказательство элементарно.

Пусть $M=\left(M_{1}, \ldots, M_{q}\right)-q$-множество. Замыканием $M$, в обозначении $\bar{M}$, называется $q$-множество $\left(\overline{M_{1}}, \ldots, \overline{M_{q}}\right)$, где $\overline{M_{i}}$ - замыкание $M_{i}$ в $\mathbb{C}^{n}$. 
Свойство 2. Пусть $\Omega$ - открытое q-множество, $A$ - q-компакт, $B$ - q-множество. Тогда $(\Omega \ominus A)+B=(\Omega \ominus A)+\bar{B}$.

Доказательство элементарно.

Свойство 3. Пусть

$$
D_{1} \supset D_{2} \supset \cdots
$$

- убьвающая последовательность q-компактов, сходящаяся к q-компакту

$$
D=\bigcap D_{n}
$$

G - открытое q-множество.

Тогда $G \ominus D=\bigcup\left(G \ominus D_{n}\right)$.

Доказательство использует вспомогательное утверждение.

Лемма. Пусть q-компакт $D$ есть предел убывающей последовательности q-компактов (1). Тогда для любого открытого q-множсества $V \supset D$ существует номер $n_{0}$ такой, что $D_{n} \subset V$ при $n \geqslant n_{0}$.

ДокАЗАТЕльство. Это утверждение следует очевидным образом из аналогичного утверждения для компактов в $\mathbb{C}^{n}$, а последнее следует из известного свойства компактов в топологических пространствах [55; следствие 3.1.5].

ДоКАЗАТЕЛЬСТВо СВОЙСТВА 3. Установим включение

$$
G \ominus D \subset \bigcup_{n=1}^{\infty}\left(G \ominus D_{n}\right)
$$

Пусть $x \in G \ominus D$, т.е. $x+D \subset G$. По условию $q$-компакт $x+D$ представляется в виде предела убывающей последовательности $q$-компактов $x+D_{1} \supset x+D_{2} \supset \cdots$. По лемме сушествует номер $n_{0}$ такой, что $x+D_{n} \subset G$ при $n \geqslant n_{0}$, или, что то же самое, $x \in G \ominus D_{n}$. Тем самым включение (2) установлено. Обратное включение следует из включений $G \ominus D_{n} \subset G \ominus D$, проистекающих из включений $D \subset D_{n}, n=$ $1,2, \ldots$ В Ваключение этого пункта отметим, что понятие геометрической разности для выпуклых множеств в $\mathbb{R}^{n}$ было введено Л. С. Понтрягиным [56].

2. $(A, B)$-выпуклость.

Пусть $A, B, \Omega$ - $q$-множества. Множество $\Omega$ назьвается $(A, B)$-вылукльлм, если справедлива импликация

$$
c \in \mathbb{C}^{n}, \quad A+c \subset \Omega \Longrightarrow B+c \subset \Omega \text {. }
$$

ЗАмЕчАнИЕ 1. Это определение подразумевает, что множество, не содержащее ни одного сдвига множества $A$, является $(A, B)$-вьпукльм.

Перечисленные ниже свойства $(A, B)$-вьпуклости достаточно элементарны и не требуют доказательства. 
Свойство 1. Пересечение любого семейства $\left\{\Omega_{\sigma}\right\}, \sigma \in \Sigma,(A, B)$-выпукльх множеств является $(A, B)$-выпуклым.

Из свойства 1 следует, что для любого множества $\Omega$ сушествует наименьшее $(A, B)$ вьпуклое множество, его содержашее; именно - пересечение всех $(A, B)$-выпуклых множеств, его содержаших. Это множество называется $(A, B)$-выпуклой оболочкой $\Omega$ и обозначается $\operatorname{conv}_{(A, B)} \Omega$.

Из замечания 1 следует: если $A+c \not \subset \Omega, \forall c \in \mathbb{C}^{n}$, то $(A, B)$-вьпуклая оболочка множества $\Omega$ совпадает с $\Omega$.

Свойство 2. Пусть $A \subset A^{\prime}$. Тогда всякое $(A, B)$-выпуклое множсество является $u\left(A^{\prime}, B\right)$-выпукльим.

СвоЙство 3. Пусть $\Omega-q$-множество и $A \subset A^{\prime}$. Тогда

$$
\operatorname{conv}_{\left(A^{\prime}, B\right)} \Omega \subset \operatorname{conv}_{(A, B)} \Omega .
$$

Свойство 4. Пусть $B \subset B^{\prime}$. Тогда всякое $\left(A, B^{\prime}\right)$-выпуклое множество является и $(A, B)$-выпукльим.

Свойство 5. Пусть $B \subset B^{\prime}$. Тогда для любого q-множества $\Omega$

$$
\operatorname{conv}_{(A, B)} \Omega \subset \operatorname{conv}_{\left(A, B^{\prime}\right)} \Omega .
$$

Свойство 6. Пусть $A$-q-компакт, $B$ - произвольное $q$-множество, $\bar{B}-$-замыкание В. Для того чтобы открытое q-множество $\Omega$ было $(A, \bar{B})$-выпуклым, необходимо и достаточно, чтобь оно было $(A, B)$-выпукльм.

Пусть $M=\left(M_{1}, \ldots, M_{q}\right)-q$-множество. Внутренняя часть $M$, в обозначении $\operatorname{Int} M$, есть $q$-множество $\left(\operatorname{Int} M_{1}, \ldots, \operatorname{Int} M_{q}\right)$, где Int $M_{i}$ - внутренняя часть $M_{i}$.

Свойство 7. Пусть $A-q$-компакт, $B$ - произвольное q-множество. Если $q$-множество $\Omega$ является $(A, B)$-выпукльим, то и Int $\Omega$ является $(A, B)$-выпукльм множсеством.

Свойство 8. Пусть $A$ - q-компакт, $B$ - произвольное q-множество, $\bar{B}$ замыкание B. Тогда для любого открытого q-множества $\Omega$

$$
\operatorname{conv}_{(A, B)} \Omega=\operatorname{conv}_{(A, \bar{B})} \Omega .
$$

3. Построение $(A, B)$-выпуклой оболочки.

Пусть $A-q$-компакт, $\Omega$ - открытое $q$-множество. Ниже приводится построение $(A, B)$-вьпуклой оболочки открытого $q$-множества $\Omega$.

Положим

$$
\begin{gathered}
\Omega_{0}=\Omega, \quad \Omega_{0}^{\prime}=\left(\Omega_{0} \ominus A\right)+B ; \\
\Omega_{1}=\Omega_{0} \cup \Omega_{0}^{\prime}, \quad \Omega_{1}^{\prime}=\left(\Omega_{1} \ominus A\right)+B ; \\
\Omega_{2}=\Omega_{1} \cup \Omega_{1}^{\prime}, \quad \Omega_{2}^{\prime}=\left(\Omega_{2} \ominus A\right)+B
\end{gathered}
$$

и т. д. Таким образом мы построим возрастающую последовательность множеств

$$
\Omega_{1} \subset \Omega_{2} \subset \cdots,
$$

где $\Omega_{n}=\Omega_{n-1} \cup \Omega_{n-1}^{\prime}, \Omega_{n-1}^{\prime}=\left(\Omega_{n-1} \ominus A\right)+B$. 
ПРЕДЛОЖЕНИЕ 5.1. (A,B)-выпуклая оболочка открытого q-множества $\Omega$ совпадает с q-множеством

$$
\Omega^{\infty}=\bigcup_{n=0}^{\infty} \Omega_{n}
$$

ДокАЗАТЕЛЬСТВо. По свойству 1 , п. 1 , геометрической разности $\Omega_{0} \ominus A$ - открытое множество; следовательно, $q$-множество $\Omega_{0}^{\prime}=\left(\Omega_{0} \ominus A\right)+B$ также открыто; открыто будет и объединение $\Omega_{1}=\Omega_{0} \cup \Omega_{0}^{\prime}$. Повторяя эти рассуждения, последовательно установим, что $q$-множества $\Omega_{2}, \Omega_{3}, \ldots$ - открыты. Следовательно, будет открыто и объединение $\Omega^{\infty}$ этих множеств. Покажем, что $q$-множество $\Omega^{\infty}$ является $(A, B)$-выпуклым. Пусть $A+c \subset \Omega^{\infty}$. Так как $A+c-q$-компакт, а $\Omega^{\infty}$ есть объединение возрастаюшей последовательности открытых $q$-множеств $\Omega_{n}$, то $A+c \subset \Omega_{n}$ для некоторого $n$. Это включение означает, что $c \in \Omega_{n} \ominus A$. Поэтому

$$
B+c \subset\left(\Omega_{n} \ominus A\right)+B=\Omega_{n}^{\prime} \subset \Omega_{n} \cup \Omega_{n}^{\prime}=\Omega_{n+1} \subset \Omega^{\infty} .
$$

Следовательно, выполняется импликация

$$
A+c \subset \Omega^{\infty} \Longrightarrow B+c \subset \Omega^{\infty},
$$

т.е. $\Omega^{\infty}$ действительно является $(A, B)$-вьпуклым. Из вьшеизложенного следует, что $(A, B)$-вьпуклая оболочка $\widehat{\Omega} q$-множества $\Omega$ содержится в $\Omega^{\infty}$. С другой стороны, из способа построения $q$-множеств $\Omega_{1}, \Omega_{2}, \ldots$ следует, что $(A, B)$-оболочка $\widehat{\Omega} q$-множества $\Omega$ содержит эти $q$-множества, значит, содержит их объединение $\Omega^{\infty}$. Тем самым, установлено, что $\widehat{\Omega}=\Omega^{\infty}$.

СлЕДСТВИЕ. Если $A$ - q-компакт, то $(A, B)$-оболочка открытого q-множества $\Omega$ открыта.

ДокАЗАТЕльСТво. Согласно предложению 5.1 указанная оболочка $\widehat{\Omega}$ представляется в виде объединения открытых множеств $\Omega_{n}, n=1,2, \ldots$.

\section{4. Предельные переходы.}

ПРЕДЛОЖЕНИЕ 5.2. Пусть $\Omega_{1} \subset \Omega_{2} \subset \cdots-$ возрастающая последовательность открытых q-множеств, сходящаяся $\kappa \Omega ; A-q$-компакт, $B$ - произвольное q-множество. Тогда

$$
\operatorname{conv}_{(A, B)} \Omega=\bigcup_{n=1}^{\infty} \operatorname{conv}_{(A, B)} \Omega_{n} .
$$

ДоКАЗАТЕЛЬСТВо. Положим

$$
\Omega^{\infty}=\bigcup_{n=1}^{\infty} \operatorname{conv}_{(A, B)} \Omega_{n} .
$$

Включение $\Omega_{n} \subset \Omega$ влечет включение

$$
\operatorname{conv}_{(A, B)} \Omega_{n} \subset \operatorname{conv}_{(A, B)} \Omega,
$$


откуда следует

$$
\Omega^{\infty} \subset \operatorname{conv}_{(A, B)} \Omega .
$$

Прежде чем установить обратное включение, покажем, что множество $\Omega^{\infty}$ является $(A, B)$-вьпуклым. По следствию к предложению $5.1 q$-множества $\operatorname{conv}_{(A, B)} \Omega_{n}$ являются открытыми. Пусть $A+c \subset \Omega^{\infty}$. Это значит, что $q$-компакт $A+c$ покрьвается возрастающей последовательностью открытых $q$-множеств $\operatorname{conv}_{(A, B)} \Omega_{n}$. Поэтому сушествует $n$ такое, что $A+c \subset \operatorname{conv}_{(A, B)} \Omega_{n}$. Поскольку $\operatorname{conv}_{(A, B)} \Omega_{n}-(A, B)$-выпуклое множество, $B+c \subset \operatorname{conv}_{(A, B)} \Omega_{n}$. Тем более, $B+c \subset \Omega^{\infty}$. Таким образом, для $\Omega^{\infty}$ выполняется импликация:

$$
A+c \subset \Omega^{\infty} \Longrightarrow B+c \subset \Omega^{\infty}
$$

т.е. $\Omega^{\infty}-(A, B)$-вьпукло. Теперь приступаем к доказательству включения, обратного (6). Имеем

$$
\Omega^{\infty}=\bigcup_{n=1}^{\infty} \operatorname{conv}_{(A, B)} \Omega_{n} \supset \bigcup_{n=1}^{\infty} \Omega_{n}=\Omega .
$$

Поскольку $q$-множество $\Omega^{\infty}$ является $(A, B)$-вьпукльм,

$$
\Omega^{\infty}=\operatorname{conv}_{(A, B)} \Omega^{\infty} \supset \operatorname{conv}_{(A, B)} \Omega
$$

Следовательно, имеет место (5). Предложение доказано.

СлЕДСТВИЕ. Пусть $A-q$-компакт, $B$ - произвольное q-множсество, $\Omega_{1} \subset$ $\Omega_{2} \subset \cdots$ - возрастающая последовательность открытых $(A, B)$-выпуклых множеств, сходящаяся $\kappa \Omega$. Тогда $\Omega$ является $(A, B)$-выпукльм.

ДокАЗАТЕЛьСтво. Поскольку каждое $\Omega_{n}$ является $(A, B)$-выпуклым,

$$
\operatorname{conv}_{(A, B)} \Omega_{n}=\Omega_{n}
$$

По предложению 5.2 имеем

$$
\operatorname{conv}_{(A, B)} \Omega=\bigcup_{n=1}^{\infty} \operatorname{conv}_{(A, B)} \Omega_{n}=\bigcup_{n=1}^{\infty} \Omega_{n}=\Omega
$$

Отсюда и следует, что $\Omega-(A, B)$-вьпукло.

ПреДЛОЖЕНИЕ 5.3. Пусть $A_{1} \supset A_{2} \supset \cdots-$ - убьвающая последовательность q-компактов, сходящаяся $\kappa A, B-q$-множество. Тогда для любого открытого q-множсества $\Omega$

$$
\operatorname{conv}_{(A, B)} \Omega=\bigcup_{n=1}^{\infty} \operatorname{conv}_{\left(A_{n}, B\right)} \Omega
$$


ДоКАЗАТЕЛЬСТво. Положим

$$
\Omega^{\infty}=\bigcup_{n=1}^{\infty} \operatorname{conv}_{\left(A_{n}, B\right)} \Omega
$$

По свойству 3 (п. 2)

$$
\operatorname{conv}_{\left(A_{n}, B\right)} \Omega \subset \operatorname{conv}_{(A, B)} \Omega, \quad n=1,2, \ldots
$$

Отсюда следует

$$
\Omega^{\infty} \subset \operatorname{conv}_{(A, B)} \Omega .
$$

Чтобы доказать обратное включение, предварительно установим, что $\Omega^{\infty}$ является $(A, B)$-вьпуклым. По следствию к предложению $5.1 q$-множества $\operatorname{conv}_{\left(A_{n}, B\right)} \Omega$ открыты и образуют возрастаюшую последовательность. Пусть $A+c \subset \Omega^{\infty}$. Поскольку $A+c$ есть $q$-компакт, покрываемый возрастаюшей последовательностью открытых $q$-множеств $\operatorname{conv}_{\left(A_{n}, B\right)} \Omega$, то $A+c \subset \operatorname{conv}_{\left(A_{n_{0}}, B\right)} \Omega$ при некотором $n_{0}$. С другой стороны, убываюшая последовательность $q$-компактов $A_{m}+c$ сходится к $A+c$. По лемме (п. 1) найдется $m \geqslant n_{0}$ столь большое, что $A_{m}+c \subset \operatorname{conv}_{\left(A_{n_{0}}, B\right)} \Omega$ и, тем более, $A_{m}+c \subset \operatorname{conv}_{\left(A_{m}, B\right)} \Omega$. Но множество $\operatorname{conv}_{\left(A_{m}, B\right)} \Omega$ является $\left(A_{m}, B\right)$-вьпуклым. Поэтому $B+c \subset \operatorname{conv}_{\left(A_{m}, B\right)} \Omega$ и, тем более, $B+c \subset \Omega^{\infty}$. Таким образом, для $q$-множества вьполняется импликация

$$
A+c \subset \Omega^{\infty} \Longrightarrow B+c \subset \Omega^{\infty}
$$

т.е. $\Omega^{\infty}-(A, B)$-выпуклое множество.

Приступим к доказательству включения, обратного (9). Используя включение

$$
\Omega \subset \operatorname{conv}_{\left(A_{n}, B\right)} \Omega
$$

и выражение (8) для $\Omega^{\infty}$, получаем включение $\Omega \subset \Omega^{\infty}$. Отсюда, используя $(A, B)$ вьпуклость $\Omega^{\infty}$, имеем

$$
\operatorname{conv}_{(A, B)} \Omega \subset \operatorname{conv}_{(A, B)} \Omega^{\infty}=\Omega^{\infty} .
$$

Это включение вместе с (9) дает (7).

ПрЕДЛОЖЕНИЕ 5.4. Пусть $A-q$-компакт; $B_{1} \subset B_{2} \subset \cdots$ - возрастающая последовательность q-множеств, сходящаяся к $B ; \Omega$ - открытое $q$-множество. Тогда

$$
\operatorname{conv}_{(A, B)} \Omega=\bigcup_{n=1}^{\infty} \operatorname{conv}_{\left(A, B_{n}\right)} \Omega
$$


ДоКАЗАТЕЛЬСТВО. Положим

$$
\Omega^{\infty}=\bigcup_{n=1}^{\infty} \operatorname{conv}_{\left(A, B_{n}\right)} \Omega .
$$

По свойству 5 (п. 2) включение $B_{n} \subset B$ влечет включение

$$
\operatorname{conv}_{\left(A, B_{n}\right)} \Omega \subset \operatorname{conv}_{(A, B)} \Omega,
$$

верное при $n=1,2, \ldots$ Отсюда следует, что

$$
\Omega^{\infty} \subset \operatorname{conv}_{(A, B)} \Omega .
$$

Установим обратное включение. Для этого покажем, что $\Omega^{\infty}$ является $(A, B)$-выпуклым. Пусть $A+c \subset \Omega^{\infty}$. По следствию к предложению $5.1 q$-множества $\operatorname{conv}_{\left(A, B_{n}\right)} \Omega$ являются открытыми и система этих множеств покрывает $q$-компакт $A+c$. Поэтому сушествует номер $n_{0}$ такой, что $A+c \subset \operatorname{conv}_{\left(A, B_{n}\right)} \Omega$ при $n \geqslant n_{0}$. Далее, так как $\left(A, B_{n}\right)$-вьпуклая оболочка любого $q$-множества является $\left(A, B_{n}\right)$-выпуклым множеством, то

$$
B_{n}+c \subset \operatorname{conv}_{\left(A, B_{n}\right)} \Omega,
$$

тем более, $B_{n}+c \subset \Omega^{\infty}$. Это верно при $n \geqslant n_{0}$. Следовательно,

$$
B+c=\bigcup_{n=n_{0}}^{\infty} B_{n}+c=\bigcup_{n=n_{0}}^{\infty}\left(B_{n}+c\right) \subset \Omega^{\infty} .
$$

Таким образом, $\Omega^{\infty}$ является $(A, B)$-вьпуклым. Так как $\Omega^{\infty} \supset \Omega$, то $(A, B)$-выпуклая оболочка $\Omega$ содержится в $\Omega^{\infty}$, т.е.

$$
\operatorname{conv}_{(A, B)} \Omega \subset \Omega^{\infty} .
$$

Тем самьм, соотношение (10) установлено.

ПРЕДЛОЖЕНИЕ 5.5. Пусть $A_{1} \supset A_{2} \supset \cdots-$ убьвающая последовательность q-компактов, сходящаяся $\kappa A ; B_{1} \subset B_{2} \subset \cdots$ - возрастающая последовательность $q$-множсеств, сходящаяся к $B ; \Omega_{1} \subset \Omega_{2} \subset \cdots$ - возрастающая последовательность открытых $q$-множеств, сходящаяся $\kappa \Omega$.

Тогда

$$
\operatorname{conv}_{(A, B)} \Omega=\bigcup \operatorname{conv}_{\left(A_{n}, B_{n}\right)} \Omega_{n} .
$$


ДОКАЗАТЕЛЬСТВО. Положим

$$
\Omega^{\infty}=\bigcup \operatorname{conv}_{\left(A_{n}, B_{n}\right)} \Omega_{n} .
$$

Включение $\Omega_{n} \subset \Omega$ влечет включение

$$
\operatorname{conv}_{\left(A_{n}, B_{n}\right)} \Omega_{n} \subset \operatorname{conv}_{\left(A_{n}, B_{n}\right)} \Omega .
$$

Так как $A \subset A_{n}$ и $B_{n} \subset B$, то, используя свойства 3,5 (п. 2), имеем

$$
\operatorname{conv}_{\left(A_{n}, B_{n}\right)} \Omega \subset \operatorname{conv}_{\left(A, B_{n}\right)} \Omega \subset \operatorname{conv}_{(A, B)} \Omega .
$$

Продолжая включение (13), получаем

$$
\operatorname{conv}_{\left(A_{n}, B_{n}\right)} \Omega_{n} \subset \operatorname{conv}_{(A, B)} \Omega .
$$

Это верно при любом $n=1,2, \ldots$ Поэтому

$$
\Omega^{\infty} \subset \operatorname{conv}_{(A, B)} \Omega .
$$

Докажем обратное включение. Фиксируем целое положительное число $m$. По свойству 3 (п. 2) при $k \geqslant m$ имеем

$$
\operatorname{conv}_{\left(A_{m}, B_{k}\right)} \Omega_{k} \subset \operatorname{conv}_{\left(A_{k}, B_{k}\right)} \Omega_{k} \subset \Omega^{\infty} .
$$

Фиксируем целое число $n \geqslant m$. По свойству 5 (п. 2 ) при $k \geqslant n$ имеем

$$
\operatorname{conv}_{\left(A_{m}, B_{n}\right)} \Omega_{k} \subset \operatorname{conv}_{\left(A_{m}, B_{k}\right)} \Omega_{k} \subset \Omega^{\infty} .
$$

Итак, $\operatorname{conv}_{A_{m}, B_{n}} \Omega_{k} \subset \Omega^{\infty}$ при $m \leqslant n \leqslant k$. Отсюда следует

$$
\bigcup_{k \geqslant n} \operatorname{conv}_{\left(A_{m}, B_{n}\right)} \Omega_{k} \subset \Omega^{\infty} \text {. }
$$

По предложению 5.2 левая часть включения есть $\operatorname{conv}_{\left(A_{m}, B_{n}\right)} \Omega$ и, следовательно,

$$
\operatorname{conv}_{\left(A_{m}, B_{n}\right)} \Omega \subset \Omega^{\infty} .
$$

Это верно при любых $m, n, m \leqslant n$. Поэтому при любом фиксированном $m=1,2, \ldots$

$$
\bigcup_{n \geqslant m} \operatorname{conv}_{\left(A_{m}, B_{n}\right)} \Omega \subset \Omega^{\infty} .
$$

По предложению 5.4 левая часть этого включения есть $\operatorname{conv}_{\left(A_{m}, B\right)} \Omega$.

Следовательно, $\operatorname{conv}_{\left(A_{m}, B\right)} \Omega \subset \Omega^{\infty}$. Поскольку это верно при $m=1,2, \ldots$,

$$
\bigcup_{m=1}^{\infty} \operatorname{conv}_{\left(A_{m}, B\right)} \Omega \subset \Omega^{\infty} .
$$

По предложению 5.3 левая часть этого включения есть $\operatorname{conv}_{(A, B)} \Omega$.

Таким образом,

$$
\operatorname{conv}_{(A, B)} \Omega \subset \Omega^{\infty} .
$$

Соотношение (11) установлено.

Предложение доказано. 


\section{§6. Проекторы Дирихле}

\section{1. Определение проектора Дирихле.}

Пусть $\Omega, \Omega^{\prime}$ - открытые $q$-множества; $H=H(\Omega), H^{\prime}=H\left(\Omega^{\prime}\right) ; W$ - инвариантное подпространство в $H$, допускающее спектральный синтез; $\Lambda$ - квазиспектральное множество подпространства $W(\S 2$, п. 6$) ; \Lambda^{\prime}$ - часть $\Lambda ; E$ - множество всех элементарных ехр- $q$-многочленов из $W$ с показателями в $\Lambda ; E^{\prime}$ - часть $E$, состоящая из всех элементарных ехр- $q$-многочленов из $E$ с показателями в $\Lambda^{\prime} ; W^{\prime}=W\left(\Lambda^{\prime}, \Omega^{\prime}\right)$ - замыкание линейной оболочки $E^{\prime}$ в топологии $H\left(\Omega^{\prime}\right)$. Согласно последнему обозначению $W=W(\Lambda, \Omega)$. Заметим, что $\Lambda^{\prime}$ - квазиспектральное множество $W^{\prime}$.

Проектором Дирихле

$$
p: W(\Lambda, \Omega) \rightarrow W\left(\Lambda^{\prime}, \Omega^{\prime}\right)
$$

мы называем линейное непрерывное отображение подпространства $W$ в подпространство $W^{\prime}$ со следуюшим свойством:

$$
p(e)= \begin{cases}e, & e \in E^{\prime}, \\ 0, & e \in E \backslash E^{\prime} .\end{cases}
$$

Так как $E$ полно в $W$, то может существовать только один проектор Дирихле.

Образ $F=p(f)$ элемента $f \in W$ относительно $p$ будем назьвать проекиией Дирихле элемента $f$. Настояший параграф посвящен исследованию свойств проектора Дирихле.

Для удобства изложения мы принимаем следующее соглашение:

если $q$-множество $\Omega^{\prime}$ пусто (т.е. хотя бы одна его компонента - пустое множество), то проектор Дирихле (1) сушествует при любых $\Lambda$ и $\Omega$.

\section{2. Проекторы Дирихле и сходимость.}

ПРЕДЛОЖЕНИЕ 6.1. Для того чтобы существовал проектор Дирихле $p: W \rightarrow$ $W^{\prime}$, необходимо и достаточно, чтобь из сходимости любой последовательности полиномов Дирихле в $W$ следовала сходимость их проекций Дирихле в $W^{\prime}$.

Доказательство столь же просто, как и в случае одной переменной [35; предложение 1.3].

\section{3. Проектор Дирихле и аналитическое продолжение.}

В случае $\Lambda^{\prime}=\Lambda$ образ $p(f)$ элемента $f$ из $W$ будем называть продолжением по Дирихле элемента $f$ на $q$-множество $\Omega^{\prime}$. Если $\Omega^{\prime}$ - q-область и $\Omega^{\prime} \supset \Omega$, то продолжение по Дирихле совпадает с обычным аналитическим продолжением.

ПреДЛОЖЕНИЕ 6.2. Пусть $\Omega \subset \Omega^{\prime}$. Для того чтобь все әлементы из $W$ допускали аналитическое продолжение в $\Omega^{\prime}$, необходимо и достаточно, чтобы они допускали продолжение по Дирихле в $\Omega^{\prime}$. 
ДоКАЗАТЕЛЬСтво. Необходимость. Пусть $\widehat{W}$ - подпространство, состоящее из элементов $H\left(\Omega^{\prime}\right)$, сужения которых на $\Omega$ принадлежат $W$. Легко проверить, что $\widehat{W}$ будет инвариантным подпространством в $H\left(\Omega^{\prime}\right)$ с тем же спектром, что и $W$. Обаподпространства $W$ и $W^{\prime}$, наделенные топологиями, индушированньми из $H(\Omega)$ и $H\left(\Omega^{\prime}\right)$ соответственно, являются пространствами типа $(\mathscr{F})$. Рассмотрим линейньй непрерьвный оператор $r: \widehat{W} \rightarrow W$ сужения элементов $\widehat{W}$ на $\Omega$. Ясно, что $\operatorname{ker} r=\{0\}$. По теореме Банаха $[57 ;$ с. 40$] r$ - топологический изоморфизм. Отсюда следует, что $\widehat{W}=W\left(\Lambda, \Omega^{\prime}\right)$ и отображение $r^{-1}$, обратное $r$, есть проектор Дирихле

$$
p: W(\Lambda, \Omega) \rightarrow W\left(\Lambda, \Omega^{\prime}\right) .
$$

Достаточность. Пусть все элементы из $W$ допускают продолжение по Дирихле в $\Omega^{\prime}$, т.е. сушествует проектор Дирихле

$$
p: W \rightarrow W\left(\Omega^{\prime}, \Lambda\right) .
$$

Фиксируем элемент $f \in W, F=p(f)$ - образ этого элемента относительно $p$. Поскольку $W$ допускает спектральньй синтез, то существует последовательность $e_{n}$, $n=1,2, \ldots$, полиномов Дирихле из $W$, сходящаяся к $f$ в $\Omega$. Так как отображение $p$ непрерьвно, последовательность $f_{n}=p\left(e_{n}\right)$ образов будет сходиться к $F$ в $\Omega^{\prime}$. Но по определяющему свойству проектора Дирихле $f_{n}=e_{n}$. Поэтому последовательность $f_{n}, n=1,2, \ldots$, будет одновременно сходиться к $F$ во всей области $\Omega^{\prime}$ и к $f$ в части $\Omega$ этой области. Отсюда следует, что сужение $F$ на $\Omega$ совпадает с $f$, т.е. $F-$ аналитическое продолжение $f$.

\section{4. Прямые суммы.}

Пусть инвариантное подпространство $W \subset H(\Omega)$ содержит инвариантные подпространства $W_{1}, \ldots, W_{n}$. Будем писать

$$
W=W_{1}+\cdots+W_{m}
$$

если $W$ представляет собою замыкание в топологии $H(\Omega)$ множества сумм

$$
f_{1}+\cdots+f_{m}, \quad f_{i} \in W_{i} .
$$

Будем говорить, что $W$ разлагается в алгебраическую прямую сумму и писать

$$
W=W_{1} \oplus \cdots \oplus W_{m}
$$

если любой элемент $f \in W$ представляется единственньм образом в виде

$$
w=w_{1}+\cdots+w_{m}, \quad \text { где } w_{i} \in W_{i} ;
$$

если при этом $w_{i}$ непрерывно зависят от $w$, то будем говорить, что $W$ разлагается в топологическую прямую сумму. 
ПреДЛОЖЕНИЕ 6.3. Алгебраическая прямая сумма $W=W_{1} \oplus \cdots \oplus W_{m}$ необходимо является топологической.

Доказательство проводится по известной схеме [35; предложение 1.5].

Пусть $W$ - инвариантное подпространство в $H$, допускаюшее спектральный синтез, $\Lambda$ - квазиспектральное множество этого подпространства. Предположим, что $\Lambda$ разбито на конечное число частей $\Lambda_{1}, \ldots, \Lambda_{m}$, т.е. $\Lambda=\bigcup_{i=1}^{m} \Lambda_{i}, \Lambda_{i} \cap \Lambda_{j}=\varnothing$ при $i \neq j$.

ПРЕДЛОЖЕНИЕ 6.4. Для того чтобы $W$ разлагалось в прямую сумму

$$
W=W_{1} \oplus \cdots \oplus W_{m},
$$

әде $W_{i}=W\left(\Lambda_{i}, \Omega\right)$, необходимо и достаточно, чтобь существовали проекторы Дирихле

$$
p_{i}: W(\Lambda, \Omega) \rightarrow W\left(\Lambda_{i}, \Omega\right), \quad i=1, \ldots, m .
$$

ДокаЗАтельство. Необходимость. Рассмотрим отображение $p_{i}: W \rightarrow W_{i}$, которое каждому элементу $w \in W$ ставит в соответствие $i$-е слагаемое в разложении $w=w_{1}+\cdots+w_{m}$. Легко проверить, что отображение $p_{i}$ будет проектором Дирихле (3).

Достаточность. Любой элемент $w \in W$ представляется в виде $w=w_{1}+\cdots+w_{m}$, где $w_{i}=p_{i}(w) \in W_{i}$ и непрерьвно зависит от $w$.

5. Перестановочность с операторами частного дифференцирования.

Из определения проектора Дирихле

$$
p: W(\Lambda, \Omega) \rightarrow W\left(\Lambda^{\prime}, \Omega^{\prime}\right)
$$

следует, что $p$ перестановочен с операторами частного дифференцирования $D_{1}=\frac{\partial}{\partial z_{1}}$, $\ldots, D_{n}=\frac{\partial}{\partial z_{n}}$ на множестве $E$ элементарных ехр- $q$-многочленов из $W$ с показателями $\lambda \in \Lambda$. Так как $E$ полно в $W$, то по непрерывности $p$ перестановочен с $D_{1}, \ldots, D_{n}$ на всем $W$.

ПреДЛОЖЕНИЕ 6.5. Пусть существует проектор Дирихле

$$
p: W(\Lambda, \Omega) \rightarrow W\left(\Lambda^{\prime}, \Omega^{\prime}\right) .
$$

Тогда для любого множества $A \subset \mathbb{C}^{n}$ существует проектор Дирихле

$$
p_{A}: W(\Lambda, \Omega+A) \rightarrow W\left(\Lambda^{\prime}, \Omega^{\prime}+A\right) .
$$

ДокАЗАТЕЛЬСтво. Введем обозначения: $W=W(\Lambda, \Omega), W^{\prime}=W\left(\Lambda^{\prime}, \Omega^{\prime}\right)$, $W_{A}=W(\Lambda, \Omega+A), W_{A}^{\prime}=W\left(\Lambda^{\prime}, \Omega^{\prime}+A\right)$. Пусть $E-$ множество ехр- $q$-многочленов из $W$ с показателями $\lambda \in \Lambda, E^{\prime}-$ множество ехр-q-многочленов из $W$ с показателями $\lambda \in \Lambda^{\prime}$. Согласно предложению 3.6 существует линейное непрерьвное отображение $v: W_{A} \rightarrow W_{A}^{\prime}$, перестановочное с операторами дифференцирования и удовлетворяюшее условию: $v(e)=p(e), \forall e \in E$. Отсюда следует

$$
v(e)= \begin{cases}e, & e \in E^{\prime} \\ 0, & e \in E \backslash E^{\prime}\end{cases}
$$

Тем самьм установлено, что $v$ - искомый проектор Дирихле (4).

Из предложения 6.5 следуют два утверждения. 
ПРЕДЛОЖЕНИЕ 6.6. Если все әлементы из $W=W(\Lambda, \Omega)$ допускают продолжение по Дирихле на $\Omega^{\prime}$, то все элементы из $W_{A}=W(\Lambda, \Omega+A)$ допускают продолжение по Дирихле на $\Omega^{\prime}+A$.

ПРЕДЛОЖЕНИЕ 6.7. Если $W$ разлагается в прямую сумму

$$
W=W_{1} \oplus \cdots \oplus W_{m}
$$

где $W_{i}=W\left(\Lambda_{i}, \Omega\right)$, то $W_{A}=W(\Lambda, \Omega+A)$ разлагается в прямую сумму

$$
W_{A}=W_{1 A} \oplus \cdots \oplus W_{m A}
$$

əде $W_{i A}=W\left(\Lambda_{i}, \Omega+A\right)$.

6. Элементарные свойства.

ПРЕДЛОЖЕНИЕ 6.8. Пусть $\Omega^{\prime \prime} \subset \Omega^{\prime}$. Тогда если существует проектор Дирихле

$$
p^{\prime}: W(\Lambda, \Omega) \rightarrow W\left(\Lambda^{\prime}, \Omega^{\prime}\right),
$$

то существует и проектор Дирихле

$$
p^{\prime \prime}: W(\Lambda, \Omega) \rightarrow W\left(\Lambda^{\prime}, \Omega^{\prime \prime}\right)
$$

ДокАЗАТЕЛЬство. Пусть $r$ - оператор сужения на $\Omega^{\prime \prime}$. Отображение $r \circ p^{\prime}$ есть искомьй проектор Дирихле $p^{\prime \prime}$.

ПРЕДЛОЖЕНИЕ 6.9. Пусть $\Lambda_{0} \supset \Lambda_{1} \supset \cdots \supset \Lambda_{n} u$

$$
\begin{aligned}
& p_{1}: W\left(\Lambda_{0}, \Omega_{0}\right) \rightarrow W\left(\Lambda_{1}, \Omega_{1}\right), \\
& p_{2}: W\left(\Lambda_{1}, \Omega_{1}\right) \rightarrow W\left(\Lambda_{2}, \Omega_{2}\right), \\
& \ldots \ldots \ldots \ldots \ldots \ldots \ldots \ldots \ldots \ldots \ldots \ldots \ldots \ldots \ldots \\
& p_{n}: W\left(\Lambda_{n-1}, \Omega_{n-1}\right) \rightarrow W\left(\Lambda_{n}, \Omega_{n}\right)
\end{aligned}
$$

- проекторы Дирихле.

Тогда композиция

$$
p=p_{n} \circ p_{n-1} \circ \cdots \circ p_{1}
$$

есть проектор Дирихле из $W\left(\Lambda_{0}, \Omega_{0}\right)$ в $W\left(\Lambda_{n}, \Omega_{n}\right)$.

ДокаЗАтельство. Оператор $р$ линейно и непрерьвно отображает $W\left(\Lambda_{0}, \Omega_{0}\right)$ в $W\left(\Lambda_{n}, \Omega_{n}\right)$, при этом элементарньй ехр- $q$-многочлен из $W\left(\Lambda_{0}, \Omega_{0}\right)$ с показателем $\lambda \in \Lambda_{n}$ остается неподвижным, а с показателем $\lambda \in \Lambda_{0} \backslash \Lambda_{n}$ переводится в нуль.

СлЕДСТВИЕ. Пусть существует проектор Дирихле $p: W\left(\Lambda, \Omega_{1}\right) \rightarrow W\left(\Lambda^{\prime}, \Omega_{2}\right)$ u $\Omega_{1} \subset \Omega_{0}, \Omega_{3} \subset \Omega_{2}$. Тогда существует проектор Дирихле

$$
p: W\left(\Lambda, \Omega_{0}\right) \rightarrow W\left(\Lambda^{\prime}, \Omega_{3}\right) .
$$


ДокАЗАТЕЛЬСТво. Проектор (5) представляется в виде $\widehat{p}=r_{2} \circ p \circ r_{1}$, где $r_{1}, r_{3}$ - отображения сужения на $\Omega_{1}, \Omega_{2}$.

ПрЕДЛОЖЕНИЕ 6.10. Пусть $\Omega_{1}, \ldots, \Omega_{m}$ - открытые q-множества и существуют проекторы Дирихле

$$
p_{i}: W(\Lambda, \Omega) \rightarrow W\left(\Lambda^{\prime}, \Omega_{i}\right), \quad i=1, \ldots, m .
$$

Тогда существует проектор Дирихле

$$
p: W(\Lambda, \Omega) \rightarrow W\left(\Lambda^{\prime}, \Omega^{\prime}\right),
$$

əде $\Omega^{\prime}=\bigcup_{i=1}^{m} \Omega_{i}$.

ДоКАЗАТЕЛЬСТВО. Для сокрашения записи обозначим: $W=W(\Lambda, \Omega), W_{i}^{\prime}=$ $W\left(\Lambda^{\prime}, \Omega_{i}\right), W^{\prime}=W\left(\Lambda^{\prime}, \Omega^{\prime}\right), W^{\prime \prime}=\bigcap_{i=1}^{m} W_{i}^{\prime}$. Нетрудно видеть, что $W^{\prime} \subset W^{\prime \prime} ;$ при этом топология $W^{\prime}$ совпадает с топологией, индуцированной из $W^{\prime \prime}$. Из определяющего свойства (2) проектора Дирихле следует, что отображения $p_{i}$ совпадают на множестве $E$ элементарных ехр- $q$-многочленов из $W$; поскольку $E$ полно в $W$, по соображениям непрерывности $p_{i}$ совпадают на $W$ и, следовательно, представляют одно и то же отображение $p$, действующее из $W$ в $W^{\prime \prime}$. Отображение $p: W \rightarrow W^{\prime \prime}$ непрерывно по той причине, что непрерывно каждое из отображений $p_{i}: W \rightarrow W_{i}^{\prime}$, и при этом топология $W^{\prime \prime}$ - слабейшая топология, при которой вложения $W^{\prime \prime} \subset W_{i}^{\prime}$, $i=1, \ldots, m$, непрерьвны.

Пусть $E^{\prime}$ - множество элементарных ехр- $q$-многочленов из $W$ с показателями в $\Lambda^{\prime}$. Из соотношений $p=p_{i}, i=1, \ldots, m$, и свойств проектора Дирихле следует

$$
p(e)= \begin{cases}e, & e \in E^{\prime}, \\ 0, & e \in E \backslash E^{\prime} .\end{cases}
$$

Для завершения доказательства нужно показать, что

$$
p(W) \subset W^{\prime} .
$$

Пусть $F=p(f)$. Сушествует последовательность $f_{n}, n=1,2, \ldots$, полиномов Дирихле с показателями в $\Lambda$, сходящаяся к $f$ в $W$. Элементы $f_{n}^{\prime}=p\left(f_{n}\right)$ суть полиномы Дирихле из $W^{\prime}$. В силу непрерьвности отображения $p: W \rightarrow W^{\prime \prime}$ последовательность $f_{n}^{\prime}, n=1,2, \ldots$, будет сходиться к $F$ в топологии $W^{\prime \prime}$. Но топология $W^{\prime}$ совпадает с топологией, индуцированной из $W^{\prime \prime}$. Значит, $f_{n}^{\prime} \rightarrow F$ в $W^{\prime}$. Так как $W^{\prime}$ замкнуто в $W^{\prime \prime}$, то $F \in W^{\prime}$. Тем самьм, включение (6) установлено.

\section{7. Предельный переход.}

ПреДЛОЖЕНИЕ 6.11. Пусть $\Omega_{1} \subset \Omega_{2} \subset \cdots$ - возрастающая последовательность открытых q-множеств, $\Omega$ - открытое q-множество. Предположим, что существуют проекторы Дирихле

$$
p_{n}: W(\Lambda, \Omega) \rightarrow W\left(\Lambda^{\prime}, \Omega_{n}\right), \quad n=1,2, \ldots .
$$

Тогда существует проектор Дирихле

$$
p: W(\Lambda, \Omega) \rightarrow W\left(\Lambda^{\prime}, \Omega^{\prime}\right),
$$

əде $\Omega^{\prime}=\bigcup_{n=1}^{\infty} \Omega_{n}$. 
ДокАЗАТЕЛьство. Для сокрашения обозначений положим $W=W(\Lambda, \Omega), W_{n}=$ $W\left(\Lambda^{\prime}, \Omega_{n}\right), W^{\prime}=W\left(\Lambda^{\prime}, \Omega^{\prime}\right)$. Можем рассматривать последовательность $W_{n}, n=$ $1,2, \ldots$, как убывающую цепочку вложенных друг в друга пространств

$$
W_{1} \supset W_{2} \supset \cdots,
$$

причем вложение $W_{n+1} \subset W_{n}$ означает, что каждый элемент $f$ из $W_{n+1}$ отождествляется со своим сужением на $\Omega_{n}$. При такой трактовке вложений (7) можно рассматривать пространство $W^{\prime}$ как пересечение: $W^{\prime}=\bigcap W_{n}$.

Пространство $W^{\prime}$ представляет собою проективньй предел последовательности пространств (7). Покажем, что для элемента $f \in W$ образ $F_{n}=p_{n}(f)$ не зависит от $n$ и принадлежит $W^{\prime}$.

Пусть $e_{\nu}, \nu=1,2, \ldots,-$ последовательность полиномов Дирихле из $W$ с показателями в $\Lambda$, сходяшаяся к $f$ в $W$. В силу непрерьвности проекторов Дирихле последовательность $p_{n}\left(e_{\nu}\right)$ будет сходиться к $F_{n}$ в топологии $W_{n}$ при $\nu \rightarrow \infty$. Но элемент $p_{n}\left(e_{\nu}\right)$ представляет собою полином Дирихле (из $W_{n}$ ), который получается из $e_{\nu}$ отбрасыванием членов с показателями в $\Lambda \backslash \Lambda^{\prime}$. Этот полином не зависит от $n$. Итак, одна и та же последовательность полиномов Дирихле сходится к $F_{n}$ в топологии $W_{n}$ при любом $n=1,2, \ldots$. Значит, эта последовательность сходится равномерно внутри $\Omega$ к некоторому элементу $F \in W$, причем этот элемент является аналитическим продолжением любого из элементов $F_{n}$. Таким образом, $F_{n}=F$ и $F \in W^{\prime}$. Тем самым установлено, что проекторы $p_{n}$ не зависят от $n$ и фактически представляют собою одно и то же отображение $p: W \rightarrow W^{\prime}$. При каждом $n$ отображение $p=p_{n}$ есть непрерывное отображение $W$ в $W_{n}$. По известному свойству проективных пределов [58; гл. V] $p$ будет непрерьвньм отображением $W$ в $W^{\prime}$. Очевидно, что отображение $p$, так же как и любое $p_{n}$, переводит элементарные exp- $q$-многочлены из $W$ с показателями в $\Lambda^{\prime}$ в самих себя, а с показателями в $\Lambda \backslash \Lambda^{\prime}-$ в нуль. Это значит, что $p: W \rightarrow W^{\prime}$-проектор Дирихле.

ПРЕДЛОЖЕНИЕ 6.12. Пусть $\Omega_{n}, n=1,2, \ldots$, - открытые q-множества и для каждого п существует проектор Дирихле

$$
p_{n}: W(\Lambda, \Omega) \rightarrow W\left(\Lambda^{\prime}, \Omega_{n}\right) .
$$

Тогда существует проектор Дирихле

$$
p: W(\Lambda, \Omega) \rightarrow W\left(\Lambda^{\prime}, \Omega^{\prime}\right)
$$

где $\Omega^{\prime}=\bigcup_{n=1}^{\infty} \Omega_{n}$.

ДокАЗАТЕЛЬСТВо. Пусть $\Omega_{m}^{\prime}=\bigcup_{n=1}^{m} \Omega_{n}$. Согласно предложению 6.10 существуют проекторы Дирихле

$$
p_{m}^{\prime}: W(\Lambda, \Omega) \rightarrow W\left(\Lambda^{\prime}, \Omega_{m}^{\prime}\right), \quad m=1,2, \ldots
$$

Далее, открытые $q$-множества $\Omega_{m}^{\prime}$ образуют возрастаюшую последовательность $\Omega_{1}^{\prime} \subset \Omega_{2}^{\prime} \subset \cdots$. По предложению 6.11 существует проектор Дирихле

$$
p: W(\Lambda, \Omega) \rightarrow W\left(\Lambda^{\prime}, \Omega^{\prime}\right),
$$

где $\Omega^{\prime}=\bigcup_{m=1}^{\infty} \Omega_{m}^{\prime}=\bigcup_{n=1}^{\infty} \Omega_{n}$. 


\section{8. Проекторы Дирихле и нестандартная выпуклость.}

ПРЕДЛОЖЕНИЕ 6.13. Пусть $D, D^{\prime}$ - открытые q-множества. Предположсим, что существует проектор Дирихле

$$
p: W(\Lambda, D) \rightarrow W\left(\Lambda^{\prime}, D^{\prime}\right)
$$

Тогда для любого открытого q-множества $\Omega$ существует проектор Дирихле

$$
\widehat{p}: W(\Lambda, \Omega) \rightarrow W\left(\Lambda^{\prime}, \Omega^{\prime}\right)
$$

где $\Omega^{\prime}=(\Omega \ominus D)+D^{\prime}$.

ДоКАЗАТЕльство. Если $\Omega \ominus D$ пусто, то и $(\Omega \ominus D)+D^{\prime}$ пусто. Согласно принятому соглашению (см. п. 1) проектор Дирихле (9) сушествует. Пусть $\Omega \ominus D$ не пусто. Положим $A=\Omega \ominus D$. По предложению 6.5 существование проектора Дирихле (8) влечет существование проектора Дирихле

$$
p_{A}: W(\Lambda, D+A) \rightarrow W\left(\Lambda^{\prime}, D^{\prime}+A\right) .
$$

Теперь заметим, что из определения геометрической разности следует $D+A \subset \Omega$. Кроме того, $\Omega^{\prime}=D^{\prime}+A$. Применяя следствие предложения 6.9 к множествам $\Omega_{0}=\Omega$, $\Omega_{1}=D+A, \Omega_{2}=D^{\prime}+A, \Omega_{3}=D^{\prime}+A$, получим, что сушествует проектор Дирихле (9).

ПРЕДЛОЖЕНИЕ 6.14. Пусть $D_{1} \ni D_{2} \ni \cdots-$ последовательность компактно вложенных открытых q-множсеств, сходящаяся к q-компакту $D ; D^{\prime}$ - открытое q-множество. Предположим, что для каждого $n=1,2, \ldots$ существует проектор Дирихле

$$
p_{n}: W\left(\Lambda, D_{n}\right) \rightarrow W\left(\Lambda^{\prime}, D^{\prime}\right) .
$$

Тогда для любого открытого q-множества $\Omega$ существует проектор Дирихле

$$
p: W(\Lambda, \Omega) \rightarrow W\left(\Lambda^{\prime}, \Omega^{\prime}\right)
$$

əде $\Omega^{\prime}=(\Omega \ominus D)+D^{\prime}$.

ДоКАЗАТЕЛЬСТВо. По предложению 6.13 для каждого $n=1,2, \ldots$ существует проектор Дирихле

$$
p_{n}: W(\Lambda, \Omega) \rightarrow W\left(\Lambda^{\prime}, \Omega_{n}^{\prime}\right),
$$

где $\Omega_{n}^{\prime}=\left(\Omega \ominus D_{n}\right)+D^{\prime}$. Пусть $\bar{D}_{n}-$ замыкание $D_{n}, \Omega_{n}^{\prime \prime}=\left(\Omega \ominus \bar{D}_{n}\right)+D^{\prime}$. Легко видеть, что $\Omega_{n}^{\prime \prime} \subset \Omega_{n}^{\prime}$. По предложению 6.8 сушествуют проекторы Дирихле

$$
p_{n}^{\prime \prime}: W(\Lambda, \Omega) \rightarrow W\left(\Lambda^{\prime}, \Omega_{n}^{\prime \prime}\right) .
$$

Заметим, что $q$-компакты $\bar{D}_{n}$ образуют убывающую последовательность, сходящуюся к $D$. По свойству 3 геометрической разности $(\S 5$, п. 1) последовательность открытых $q$-множеств $\Omega_{n}^{\prime \prime}, n=1,2, \ldots$, возрастает и сходится к $\Omega^{\prime}$. Согласно предложению 6.11 существует проектор Дирихле (10). 
ПРЕДЛОЖЕНИЕ 6.15. Пусть $D_{1} \ni D_{2} \ni \cdots-$ - убывающая последовательность компактно вложенных друг в друга открытых q-множеств, сходящаяся $к$ q-компакту $D ; D^{\prime}$ - открытое q-множество. Предположим, что для каждого $n=1,2, \ldots$ существует проектор Дирихле

$$
p_{n}: W\left(\Lambda, D_{n}\right) \rightarrow W\left(\Lambda^{\prime}, D^{\prime}\right), \quad n=1,2, \ldots
$$

Тогда для любого открытого q-множества $\Omega$ существует проектор Дирихле

$$
p: W(\Lambda, \Omega) \rightarrow W\left(\Lambda^{\prime}, \Omega^{\prime}\right)
$$

где $\Omega^{\prime}-\left(D, D^{\prime}\right)$-вьпукклая оболочка $\Omega$, если $\Lambda=\Lambda^{\prime}$, и $\Omega^{\prime}-\left(D, D^{\prime}\right)$-вьлпукля оболочка $(\Omega \ominus D)+D^{\prime}$, если $\Lambda^{\prime} \subset \Lambda$.

ЗАмЕЧАНИЕ. $q$-множества $\Omega$ и $(\Omega \ominus D)+D^{\prime}$ являются открытыми; поэтому по свойству 8 п. $2 \S 5,\left(D, D^{\prime}\right)$-вьпуклые оболочки этих множеств совпадают с их $\left(D, \bar{D}^{\prime}\right)$-выпуклыми оболочками, где $\bar{D}^{\prime}-$ замыкание $D^{\prime}$.

ДокАЗАтельство. Рассмотрим случай $\Lambda^{\prime}=\Lambda$. Построим возрастаюшую последовательность открытых $q$-множеств

$$
\Omega_{0} \subset \Omega_{1} \subset \cdots,
$$

где $\Omega_{0}=\Omega, \Omega_{1}=\Omega_{0} \cup \Omega_{0}^{\prime}, \Omega_{0}^{\prime}=\left(\Omega_{0} \ominus D\right)+D^{\prime}, \Omega_{2}=\Omega_{1} \cup \Omega_{1}^{\prime}, \Omega_{1}^{\prime}=\left(\Omega_{1} \ominus D\right)+D^{\prime}$ и т. д.

Таким образом, в последовательности (12) $\Omega_{n}=\Omega_{n-1} \cup \Omega_{n-1}^{\prime}, \quad \Omega_{n-1}^{\prime}=$ $\left(\Omega_{n-1} \ominus D\right)+D^{\prime}$. Согласно предложению $5.1\left(D, D^{\prime}\right)$-выпуклая оболочка $\Omega$ совпадает с $\Omega^{\prime}=\bigcup_{n=0}^{\infty} \Omega_{n}$. По предложению 6.14 сушествует проектор Дирихле

$$
p_{0}^{\prime}: W\left(\Lambda, \Omega_{0}\right) \rightarrow W\left(\Lambda, \Omega_{0}^{\prime}\right)
$$

Тождественное отображение

$$
p_{0}^{\prime \prime}: W\left(\Lambda, \Omega_{0}\right) \rightarrow W\left(\Lambda, \Omega_{0}\right)
$$

есть проектор Дирихле. Отсюда, согласно предложению 6.10, заключаем, что сушествует проектор Дирихле

$$
p_{1}: W\left(\Lambda, \Omega_{0}\right) \rightarrow W\left(\Lambda, \Omega_{1}\right) .
$$

Применяя снова предложение 6.14 к $q$-множеству $\Omega_{1}$, получим, что существует проектор Дирихле

$$
p_{1}^{\prime}: W\left(\Lambda, \Omega_{1}\right) \rightarrow W\left(\Lambda, \Omega_{1}^{\prime}\right) .
$$

Обозначим $p_{1}^{\prime \prime}: W\left(\Lambda, \Omega_{1}\right) \rightarrow W\left(\Lambda, \Omega_{1}\right)$ тождественное отображение. Это отображение является проектором Дирихле. По предложению 6.10 сушествует проектор Дирихле

$$
p_{2}: W\left(\Lambda, \Omega_{1}\right) \rightarrow W\left(\Lambda, \Omega_{2}\right)
$$


Последовательно проводя такие рассуждения, получим, что для каждого $n=1,2, \ldots$ сушествует проектор Дирихле

$$
p_{n}: W\left(\Lambda, \Omega_{n-1}\right) \rightarrow W\left(\Lambda, \Omega_{n}\right) .
$$

Рассмотрим суперпозицию $\widehat{p}_{n}=p_{n} \circ p_{n-1} \circ \cdots \circ p_{1} . \widehat{p}_{n}$ есть линейное непрерьвное отображение пространства $W(\Lambda, \Omega)$ в $W\left(\Lambda, \Omega_{n}\right)$ По предложению $6.9 \widehat{p}_{n}$ - проектор Дирихле.

Итак, для каждого $n=1,2, \ldots$ сушествует проектор Дирихле

$$
\widehat{p}_{n}: W(\Lambda, \Omega) \rightarrow W\left(\Lambda, \Omega_{n}\right) .
$$

Так как $q$-множества $\Omega_{n}$ образуют возрастаюшую последовательность, сходящуюся к $\Omega^{\prime}$, то по предложению 6.11 , сушествует проектор Дирихле

$$
p: W(\Lambda, \Omega) \rightarrow W\left(\Lambda, \Omega^{\prime}\right) .
$$

В случае $\Lambda^{\prime}=\Lambda$ утверждение предложения доказано.

Рассмотрим случай $\Lambda^{\prime} \neq \Lambda$. По предложению 6.14 существует проектор Дирихле

$$
\widehat{p}: W(\Lambda, \Omega) \rightarrow W\left(\Lambda^{\prime}, \widehat{\Omega}\right),
$$

где $\widehat{\Omega}=(\Omega \ominus D)+D^{\prime}$. Теперь заметим, что условие (11) влечет существование проекторов

$$
\widehat{p}_{n}: W\left(\Lambda^{\prime}, D_{n}\right) \rightarrow W\left(\Lambda^{\prime}, D^{\prime}\right), \quad n=1,2, \ldots
$$

Это позволяет свести случай $\Lambda^{\prime} \neq \Lambda$ к уже разобранному случаю $\Lambda^{\prime}=\Lambda$ (с заменой $\Lambda$ на $\left.\Lambda^{\prime}\right)$. В силу (13), по уже доказанному, существует проектор Дирихле

$$
p^{\prime}: W\left(\Lambda^{\prime}, \widehat{\Omega}\right) \rightarrow W\left(\Lambda^{\prime}, \Omega^{\prime}\right),
$$

где $\Omega^{\prime}-\left(D, D^{\prime}\right)$-вьпуклая оболочка $\widehat{\Omega}$. По предложению 6.9 суперпозиция

$$
p=p^{\prime} \circ \widehat{p}
$$

есть проектор Дирихле, отображаюший $W(\Lambda, \Omega)$ в $W\left(\Lambda^{\prime}, \Omega^{\prime}\right)$. Предложение доказано.

\section{$\S$ 7. Мономорфизмы Дирихле}

1. Пусть $\Omega, \Omega^{\prime}$ - открытые $q$-множества; $P, P^{\prime}$ - модули, ассоциированные с $\Omega, \Omega^{\prime}$ соответственно; $I, I^{\prime}$ - обильные подмодули в $P, P^{\prime} ; \Lambda, \Lambda^{\prime}$ - квазинулевые множества подмодулей $I, I^{\prime}$ соответственно (см. $\S 2$, п. 6). Предположим, что $\Lambda^{\prime} \subset \Lambda$ и

$$
I^{\prime}(\lambda)=I(\lambda) \quad \forall \lambda \in \Lambda^{\prime} .
$$

Мономорфизмом, Дирихле назьвается линейное отображение

$$
u: P^{\prime} / I^{\prime} \rightarrow P / I,
$$


которое класс смежности $\Psi \in P^{\prime} / I^{\prime}$ переводит в класс смежности $\Phi \in P / I$ так, что вьполняются условия

$$
\begin{aligned}
& \Phi \equiv \Psi(\bmod I(\lambda)) \quad \forall \lambda \in \Lambda^{\prime}, \\
& \Phi \equiv 0(\bmod I(\lambda)) \quad \forall \lambda \in \Lambda \backslash \Lambda^{\prime} .
\end{aligned}
$$

ЗАмЕчАНИЕ. Условия (1), (2) означают, что для любых представителей $\varphi, \psi$ классов $\Phi, \Psi$ имеют место сравнения $\varphi \equiv \psi(\bmod I(\lambda))$ при $\lambda \in \Lambda^{\prime}$ и $\varphi \equiv 0(\bmod I(\lambda))$ при $\lambda \in \Lambda \backslash \Lambda^{\prime}$.

Отметим, что может сушествовать не более одного класса $\Phi \in P / I$, удовлетворяющего условиям (1), (2). Действительно, если существует другой класс $\Phi^{\prime}$, то для любых двух элементов $\varphi \in \Phi, \varphi^{\prime} \in \Phi^{\prime}$ будет $\varphi-\varphi^{\prime} \equiv 0(\bmod I(\lambda)) \forall \lambda \in \Lambda$. Отсюда, в силу того, что подмодуль $I$ - обильный, а $\Lambda$ - квазинулевое множество этого подмодуля, справедливо включение $\varphi-\varphi^{\prime} \in I$. Это значит, что $\Phi=\Phi^{\prime}$. Аналогичньм образом проверяется, что отображение $u$ мономорфно.

ПРЕДЛОЖЕНИЕ 7.1. Для того чтобы существовал проектор Дирихле

$$
p: W(\Lambda, \Omega) \rightarrow W\left(\Lambda^{\prime}, \Omega^{\prime}\right),
$$

необходимо и достаточно, чтобы существовал непрерывный мономорфизм Дирихле

$$
u: P^{\prime} / I^{\prime} \rightarrow P / I .
$$

ДокаЗАтельство. Необходимость. Пусть сушествует проектор Дирихле (3). Рассмотрим дуальную операцию $u=p^{\circledast}: P^{\prime} / I^{\prime} \rightarrow P / I$. Для многочлена $P \in \operatorname{Sp}_{\lambda} W$ имеем

$$
p(P(z) \exp \langle\lambda, z\rangle)= \begin{cases}P(z) \exp \langle\lambda, z\rangle, & \lambda \in \Lambda^{\prime}, \\ 0, & \lambda \in \Lambda \backslash \Lambda^{\prime} .\end{cases}
$$

Отсюда, в силу предложения 3.5 для класса смежности $\Psi \in P^{\prime} / I^{\prime}$ и его образа $\Phi=p^{\circledast}(\Psi)$ имеем

$$
\left.P(D) \Phi\right|_{\lambda}= \begin{cases}\left.P(D) \Psi\right|_{\lambda} & \forall \lambda \in \Lambda^{\prime}, \\ 0 & \forall \lambda \in \Lambda \backslash \Lambda^{\prime} .\end{cases}
$$

При фиксированном $\lambda$ соотношения (5) справедливы для любого многочлена $P$ из локального спектра $\mathrm{Sp}_{\lambda} W$. Но согласно предложению $2.1 \mathrm{Sp}_{\lambda} W=\operatorname{Div}_{\lambda} I$. Поэтому соотношения (5) можно записать в виде

$$
\begin{gathered}
\left.P(D)(\Phi-\Psi)\right|_{\lambda}=0 \quad \forall P \in \operatorname{Div}_{\lambda} I \quad \forall \lambda \in \Lambda^{\prime}, \\
\left.P(D) \Phi\right|_{\lambda}=0 \quad \forall P \in \operatorname{Div}_{\lambda} I \quad \forall \lambda \in \Lambda \backslash \Lambda^{\prime} .
\end{gathered}
$$

По свойству 8 дивизора (см. $\S 2$, п. 4 )

$$
\begin{aligned}
& \Phi-\Psi \subset I(\lambda) \quad \forall \lambda \in \Lambda^{\prime}, \\
& \Phi \subset I(\lambda) \quad \forall \lambda \in \Lambda \backslash \Lambda^{\prime} .
\end{aligned}
$$


Тем самьм установлено, что отображение $u=p^{\circledast}$ есть непрерывный мономорфизм Дирихле.

Достаточность. Пусть существует непрерывный мономорфизм Дирихле (4). Известно (см. $\S 3$, п. 1 ), что существует линейная непрерывная операция $p: W \rightarrow W^{\prime}$, по отношению к которой $u$ является дуальной операцией: $u=p^{\circledast}$. Покажем, что $p$ - проектор Дирихле. Отображение $u$ переводит класс смежности $\Psi \in P^{\prime} / I^{\prime}$ в класс смежности $\Phi \in P / I$, связанный с $\Psi$ соотношениями (1), (2). В силу свойства 8 дивизора $(\S 2$, п. 4$)$ эти соотношения можно переписать в виде

$$
\begin{gathered}
P(D) \Phi=\left.P(D) \Psi\right|_{\lambda} \quad \forall P \in \operatorname{Div}_{\lambda} I \quad \forall \lambda \in \Lambda^{\prime}, \\
P(D) \Phi=0 \quad \forall P \in \operatorname{Div}_{\lambda} I \quad \forall \lambda \in \Lambda \backslash \Lambda^{\prime} .
\end{gathered}
$$

Применяя предложение 3.5 , видим, что прямая операция $p$ действует на элементарный ехр- $q$-многочлен

$$
e=P(z) \exp \langle\lambda, z\rangle \in W
$$

следуюшим образом:

$$
p(e)= \begin{cases}e, & \text { если } \lambda \in \Lambda^{\prime}, \\ 0, & \text { если } \lambda \in \Lambda \backslash \Lambda^{\prime} .\end{cases}
$$

Это значит, что $p$ - проектор Дирихле.

2. Введем следуюшие обозначения. Для вьпуклого $q$-компакта $K=\left(K_{1}, \ldots, K_{q}\right)$ и целой $q$-функции $\varphi=\left(\varphi_{1}, \ldots, \varphi_{q}\right)$ положим

$$
\|\varphi\|_{K}=\sum_{i=1}^{q} \sup _{z \in \mathbb{C}^{n}} \frac{\left|\varphi_{i}(\lambda)\right|}{\exp H_{K_{i}}(\lambda)}
$$

где $H_{K_{i}}(\lambda)=\sup _{\zeta \in K_{i}} \operatorname{Re}\langle\zeta, \lambda\rangle$ - опорная функция $K_{i}$ в смысле комплексного анализа; $P_{K}$ - банахово пространство целых $q$-функций с нормой $\|\varphi\|_{K}<\infty ; B_{K}=\left\{\varphi \in P_{K}\right.$ : $\left.\|\varphi\|_{K} \leqslant 1\right\}$ - единичньй шар в $P_{K}$.

ПРЕДЛОЖЕНИЕ 7.2. Следующие два утверждения равносильны:

А) существует непрерьвный мономорфизм Дирихле $и: P^{\prime} / I^{\prime} \rightarrow P / I$;

Б) для любого q-компакта $K^{\prime} \subset \Omega^{\prime}$ существуют q-компакт $K \subset \Omega$ и константа $C$ такие, что для любого әлемента $f^{\prime} \in P^{\prime}$ найдется әлемент $f \in P$, удовлетворяющий условиям:

1) имеют место сравнения

$$
f \equiv f^{\prime} \quad(\bmod I(\lambda)) \quad \forall \lambda \in \Lambda^{\prime}, \quad f \equiv 0 \quad(\bmod I(\lambda)) \quad \forall \lambda \in \Lambda \backslash \Lambda^{\prime} ;
$$

2) имеет место неравенство

$$
\|f\|_{K} \leqslant C\left\|f^{\prime}\right\|_{K^{\prime}}
$$


Доказательство этого предложения требует подготовки, которую мы проведем в п. 3,4 .

3. Пусть $P$ - модуль, ассоциированньй с выпуклой $q$-областью $\Omega$. В этом пункте мы будем рассматривать $P$ как локально-вьпуклое пространство над полем комплексных чисел. Наша цель - описать фактор-топологию фактор-пространства $P / I$ по замкнутому подпространству $I \subset P$.

Пусть $K_{1} \Subset K_{2} \Subset \cdots-$ возрастаюшая последовательность вьпуклых $q$-компактов, исчерпывающая $\Omega$. Используя теорему Мартино-Эренпрайса [36; теорема 4.5.3], можно показать, что топология $P$ есть индуктивньй предел возрастаюшей последовательности

$$
P_{K_{1}} \subset P_{K_{2}} \subset \cdots
$$

банаховых пространств, вложенных друг в друга вполне непрерьвно. Напомним, что $B_{K_{n}}$ - единичный шар в $P_{K_{n}}$. Абсолютно вьпуклое множество $V \subset P$ является окрестностью нуля в $P$ тогда и только тогда, когда для любого $n=1,2, \ldots$ найдется положительная константа $c_{n}$ такая, что $c_{n} B_{K_{n}} \subset V$.

Пусть $I$ - замкнутое подпространство $P$. Рассмотрим фактор-пространство $P / I$, наделенное фактор-топологией топологии $P$ по $I$. Обозначим через $\theta$ канонический гомоморфизм $P \rightarrow P / I$. Совокупность образов $\theta(V)$ окрестностей нуля $V \subset P$ является фундаментальной системой окрестностей нуля в $P / I$. Соответствующую топологию обозначим $\tau$. Кроме $\tau$ в $P / I$ можно ввести топологию $\mu$ индуктивного предела следуюшим образом. Каждая норма $\|\cdot\|_{K_{n}}$ порождает в $P / I$ фактор-норму

$$
\|\Phi\|_{K_{n}}=\inf \left\{\|\varphi\|_{K_{n}}: \varphi \in \Phi\right\} .
$$

Обозначим $(P / I)_{K_{n}}$ нормированное пространство классов смежности $\Phi \in P / I$, для которых $\|\Phi\|_{K_{n}}<\infty$. Легко видеть, что единичный шар $\widehat{B}_{K_{n}}=\{\Phi \in P / I$ : $\left.\|\Phi\|_{K_{n}} \leqslant 1\right\}$ содержит в себе образ $\theta\left(B_{K_{n}}\right)$ единичного шара $B_{K_{n}} \subset P_{K_{n}}$ и содержится в образе того же, но удвоенного шара:

$$
\theta\left(B_{K_{n}}\right) \subset \widehat{B}_{K_{n}} \subset \theta\left(2 B_{K_{n}}\right)
$$

Очевидно, что

$$
\bigcup_{n=1}^{\infty}(P / I)_{K_{n}}=P / I .
$$

Обозначим через $\mu$ топологию индуктивного предела возрастающей последовательности нормированных пространств

$$
(P / I)_{K_{1}} \subset(P / I)_{K_{2}} \subset \cdots .
$$

ПРЕДЛОЖЕНИЕ 7.3. Фактор-топология $\tau$ пространства $P / I$ совпадает с топологией $\mu$ индуктивного предела последовательности (10); при әтом вложения в (10) являются вполне непрерывнылми, т.е. $P / I$ является пространством muna $\left(L N^{*}\right)$.

Доказательство основано на сравнении окрестностей нуля фактор-пространства $P / I$ в топологии $\tau$ и в топологии $\mu$; при сравнении используются включения (9).

Полная непрерывность вложений (10) следует тривиальным образом из полной непрерывности вложений (8).

4. Пусть $u$ - линейное отображение $P^{\prime} / I^{\prime}$ в $P / I$. Нас интересуют условия, при которых это отображение будет непрерывным. 
ПреДЛОЖенИЕ 7.4. Для того чтобы линейное отображение $u: P^{\prime} / I^{\prime} \rightarrow P / I$ было непрерывным, необходимо и достаточно, чтобы выполнялось следующее условие: для любого выпукллого q-компакта $K^{\prime} \subset \Omega^{\prime}$ существует выпуклый $q$-компакт $K \subset \Omega$ такой, что для любого әлемента $f^{\prime} \in P^{\prime}$ существует әлемент $f \in u\left(\left[f^{\prime}\right]\right)$, для которого

$$
\|f\|_{K} \leqslant \mathrm{const}\left\|f^{\prime}\right\|_{K^{\prime}}
$$

әде констанста не зависит от $f^{\prime}, f$.

ДоказАтельство. Необходимость. Пусть отображение $u$ непрерьвно. Образ $\widehat{B}_{K^{\prime}}$ шара $B_{K^{\prime}}$ относительно канонического гомоморфизма $\theta^{\prime}: P^{\prime} \rightarrow P^{\prime} / I^{\prime}$ представляет собою ограниченное множество в $P^{\prime} / I^{\prime}$. Поэтому его образ $u\left(\widehat{B}_{K^{\prime}}\right)-$ ограниченное множество в $P / I$. Согласно предложению $7.3 P / I$ есть пространство типа $\left(L N^{*}\right)$ с определяющей последовательностью нормированных пространств $(P / I)_{K_{n}}, n=1,2, \ldots$ По известному свойству таких пространств [44; теорема 2] $u\left(\widehat{B}_{K^{\prime}}\right)$ ограничено в некотором $(P / I)_{K_{n}}$. Полагая $K=K_{n}$, можем утверждать, что сушествует $q$-компакт $K \subset \Omega$ такой, что $u\left(\widehat{B}_{K^{\prime}}\right) \subset$ const $\widehat{B}_{K}$, где $\widehat{B}_{K}$ - образ шара $B_{K}$ относительно канонического гомоморфизма $\theta: P \rightarrow P / I$. Последнее равносильно тому, что

$$
\left\|u ( F ^ { \prime } ) \left|\left\|_{K} \leqslant c \mid\right\| F^{\prime} \|_{K^{\prime}}\right.\right.
$$

для любого класса $F^{\prime} \in P^{\prime} / I^{\prime}$. Здесь $c$-положительная константа. Пусть теперь $f^{\prime}$ - какой-либо элемент из $P^{\prime}$, а $F^{\prime}$ - класс смежности, его содержаший. Очевидно,

$$
\left\|F^{\prime}\right\|_{K^{\prime}} \leqslant\left\|f^{\prime}\right\|_{K^{\prime}}
$$

Выберем элемент $f$ из $u\left(F^{\prime}\right)$, для которого

$$
\|f\|_{K} \leqslant 2\left\|u\left(F^{\prime}\right)\right\|_{K} .
$$

Учитьвая (12), получим

$$
\|f\|_{K} \leqslant 2\left\|u\left(F^{\prime}\right)\right\|_{K} \leqslant 2 c\left\|f^{\prime}\right\|_{K^{\prime}} .
$$

Необходимая часть утверждения доказана.

Достаточность. Пусть $K_{1}^{\prime} \Subset K_{2}^{\prime} \Subset \cdots-$ возрастаюшая последовательность выпуклых $q$-компактов, исчерпьвающая $\Omega^{\prime}$. По предложению $7.3 P^{\prime} / I^{\prime}$ есть индуктивный предел пространств $\left(P^{\prime} / I^{\prime}\right)_{K_{n}^{\prime}}$. Пусть выполняется условие (11). Это условие можно переформулировать следуюшим образом: для любого выпуклого $q$-компакта $K^{\prime} \subset \Omega^{\prime}$ существует $q$-компакт $K \subset \Omega$ такой, что

$$
u\left(\widehat{B}_{K^{\prime}}\right) \subset \text { const } \widehat{B}_{K},
$$

где $\widehat{B}_{K^{\prime}}, \widehat{B}_{K}$ - образы шаров $B_{K^{\prime}}, B_{K}$ относительно канонических гомоморфизмов $\theta^{\prime}: P^{\prime} \rightarrow P^{\prime} / I^{\prime}, \theta: P \rightarrow P / I$ соответственно. 
Включение (13) означает, что для любого $q$-компакта $K^{\prime} \subset \Omega^{\prime}$ образ $u\left(\widehat{B}_{K^{\prime}}\right)$ ограничен в $P / I$; в частности, это верно при $K^{\prime}=K_{n}^{\prime}, n=1,2 \ldots$ Пусть $\widehat{V}$ - абсолютно вьпуклая окрестность нуля в $P / I$. Из вьшеизложенного следует, что для любого $n=1,2, \ldots$ сушествует $\varepsilon_{n}>0$ такое, что

$$
u\left(\varepsilon_{n} \widehat{B}_{K_{n}^{\prime}}\right) \subset \widehat{V}
$$

Иными словами, прообраз $V^{\prime}=u^{-1}(\widehat{V})$ окрестности $\widehat{V}$ содержит множества $\varepsilon_{n} \widehat{B}_{K_{n}^{\prime}}$, $n=1,2, \ldots$. Поскольку $V^{\prime}$ - абсолютно вьпуклое множество вместе с $\widehat{V}$, заключаем, что $V^{\prime}$ - окрестность нуля в $P^{\prime} / I^{\prime}$. Тем самым, установлено, что прообраз $V^{\prime}=$ $u^{-1}(\widehat{V})$ любой абсолютно вьпуклой окрестности $\widehat{V} \subset P / I$ есть окрестность в $P^{\prime} / I^{\prime}$, т.е. отображение $u$ непрерывно.

\section{5. Доказательство предложения 7.2.}

$1^{\circ}$. Пусть верно утверждение А), т.е. сушествует непрерывный мономорфизм Дирихле $u: P^{\prime} / I^{\prime} \rightarrow P / I$. Согласно предложению 7.4 для любого $q$-компакта $K^{\prime} \subset \Omega^{\prime}$ сушествуют $q$-компакт $K \subset \Omega$ и константа $c<\infty$ такие, что справедливо следующее утверждение:

для любого элемента $f^{\prime} \in P^{\prime}$ существует элемент $f \in P$ такой, что

$$
\begin{gathered}
f \in u\left(\left[f^{\prime}\right]\right), \\
\|f\|_{K} \leqslant c\left\|f^{\prime}\right\|_{K^{\prime}} .
\end{gathered}
$$

Поскольку $u$ - мономорфизм Дирихле, включение (14) равносильно сравнениям (6); а условие (15) совпадает с условием (7). Условие Б) вьполнено.

$2^{\circ}$. Пусть имеет место условие Б), т.е. для любой $q$-функции $f^{\prime} \in P^{\prime}$ найдется $q$-функция $f$, связанная с $f^{\prime}$ условиями (6), (7). Рассмотрим отображение $u: P^{\prime} / I^{\prime} \rightarrow$ $P / I$, которое классу смежности $\Psi=\left[f^{\prime}\right]$ ставит в соответствие класс смежности $\Phi=[f]$. Покажем, что определение корректно. Пусть $f_{0}^{\prime}$ - другой элемент из класса $\Psi$. Этому элементу соответствует элемент $f_{0}$ и класс $\Phi_{0}=\left[f_{0}\right]$ в $P / I$. Элементы $f, f_{0}$ удовлетворяют сравнениям

$$
\begin{gathered}
f \equiv \begin{cases}f^{\prime}(\bmod I(\lambda)) & \forall \lambda \in \Lambda^{\prime}, \\
0(\bmod I(\lambda)) & \forall \lambda \in \Lambda \backslash \Lambda^{\prime},\end{cases} \\
f_{0} \equiv \begin{cases}f_{0}^{\prime}(\bmod I(\lambda)) & \forall \lambda \in \Lambda^{\prime}, \\
0(\bmod I(\lambda)) & \forall \lambda \in \Lambda \backslash \Lambda^{\prime} .\end{cases}
\end{gathered}
$$

Ввиду того, что $f^{\prime} \equiv f_{0}^{\prime}(\bmod I(\lambda)) \forall \lambda \in \Lambda^{\prime}$, из этих сравнений следует, что $f-f_{0} \in$ $I(\lambda) \forall \lambda \in \Lambda$. Поскольку $I$ - обильньй подмодуль в $P$, а $\Lambda$ - квазинулевое множество подмодуля $I$, заключаем, что $f-f_{0} \in I$. Значит, $\Phi=\Phi_{0}$. Тем самым, установлено, что $u$ определено корректно. Отображение $u$ удовлетворяет условиям (14), (15). Легко проверяется линейность этого отображения. На основании предложения 7.4 заключаем, что отображение $u$ - непрерьвньй мономорфизм Дирихле.

6. В некоторых случаях мономорфизм Дирихле является непрерьвным автоматически. Следующее утверждение для случая одного переменного известно [35]. 
ПРЕДЛОЖЕНИЕ 7.5. Пусть выполнено условие а) $\Lambda=\Lambda^{\prime}$, либо условие б) $\Omega \subset \Omega^{\prime}$.

Тогда алгебраический мономорфизм Дирихле $q: P^{\prime} / I^{\prime} \rightarrow P / I$ является непрерывныцм; при этом в случае б) $q$ - топологический мономорфизм.

ЗАмечАниЕ. Сушествование мономорфизма Дирихле $q$ эквивалентно условию: для любого элемента $\psi \in P^{\prime}$ существует элемент $\varphi \in P$ такой, что

$$
\varphi \equiv \psi \quad(\bmod I(\lambda)) \quad \forall \lambda \in \Lambda
$$

в случае а) и

$$
\varphi \equiv \begin{cases}\psi(\bmod I(\lambda)) & \forall \lambda \in \Lambda^{\prime} \\ 0(\bmod I(\lambda)) & \forall \lambda \in \Lambda \backslash \Lambda^{\prime}\end{cases}
$$

в случае б).

Доказательство проводится по схеме доказательства теоремы 1.11 из статьи [35], и мы его опускаем.

\section{§ 8. Разделение множеств}

\section{1. О кратности систем.}

Пусть $E=\left\{e_{\alpha}\right\}, \alpha \in A,-$ некоторая система замкнутых шаров в $\mathbb{R}^{m}$. Кратностью системы $E$ в точке $x$, в обозначении $k_{E}(x)$, назьвается число шаров $e_{\alpha}$, содержаших $x$. Число

$$
k_{E}=\sup \left\{k_{E}(x): x \in \mathbb{R}^{m}\right\}
$$

называется кратностью системы $E$.

Рассмотрим две системы шаров

$$
E=\left\{e_{\alpha}\right\}, \quad B=\left\{b_{\alpha}\right\}, \quad \alpha \in A .
$$

Предположим, что

$$
e_{\alpha} \subset b_{\alpha} \quad \forall \alpha \in A \text {. }
$$

Введем обозначения: $\rho(b)-$ радиус шара $b, \rho_{B}^{+}(x)=\sup \left\{\rho\left(b_{\alpha}\right): x \in b_{\alpha}\right\}, \rho_{E \mid B}^{-}(x)=$ $\inf \left\{\rho\left(e_{\alpha}\right): x \in b_{\alpha}\right\}$.

ПРЕДЛОЖЕНИЕ 8.1. Пусть система Е имеет конечную кратность $k_{E}$. Тогда

$$
k_{B}(x) \leqslant k_{E} 2^{m}\left(\frac{\rho_{B}^{+}(x)}{\rho_{E \mid B}^{-}(x)}\right)^{m}
$$


ДокаЗАтельство. Фиксируем $x \in \mathbb{R}^{m}$. Пусть $B(x)=\bigcup\left\{b_{\alpha}: x \in b_{\alpha}\right\}$. Обозначим $|e|$ объем шара $e$. В силу условия (1) имеем

$$
\sum_{x \in b_{\alpha}}\left|e_{\alpha}\right| \leqslant k_{E} \int_{B(x)} d s
$$

где $d s$ - элемент меры Лебега.

Множество $B(x)$ содержится в шаре с центром в $x$ и радиуса $2 \rho_{B}^{+}(x)$. Поэтому интеграл в правой части не превосходит $B(1) 2^{m}\left(\rho_{B}^{+}(x)\right)^{m}$, где $B(1)$ - обозначение объема единичного шара. Отсюда

$$
\sum_{x \in b_{\alpha}}\left|e_{\alpha}\right| \leqslant k_{E} B(1) 2^{m}\left(\rho_{B}^{+}(x)\right)^{m} .
$$

$\mathrm{C}$ другой стороны, радиус шара $e_{\alpha}$, для которого $x \in b_{\alpha}$, не менее $\rho_{E \mid B}^{-}(x)$. Поэтому

$$
\sum_{x \in b_{\alpha}}\left|e_{\alpha}\right| \geqslant k_{B}(x) B(1)\left(\rho_{E \mid B}^{-}(x)\right)^{m} .
$$

Из (3) и (4) следует (2).

$\mathrm{B}$ дальнейшем нас будут интересовать системы шаров, заданные с помошью положительной функции $\omega(x)$. Рассмотрим системы шаров $B=\left\{b_{\alpha}\right\}, E=\left\{e_{\alpha}\right\}, \alpha \in A$, где

$$
\begin{aligned}
b_{\alpha} & =\left\{\zeta \in \mathbb{R}^{m}:\left|\zeta-x_{\alpha}\right| \leqslant \omega\left(x_{\alpha}\right)\right\}, \\
e_{\alpha} & =\left\{\zeta \in \mathbb{R}^{m}:\left|\zeta-x_{\alpha}\right| \leqslant \frac{\omega\left(x_{\alpha}\right)}{2}\right\} .
\end{aligned}
$$

Здесь $\left\{x_{\alpha}\right\}, \alpha \in A,-$ некоторая система точек в $\mathbb{R}^{m}$.

Пусть

$$
\begin{aligned}
& \omega^{+}(x)=\sup \{\omega(\zeta):|\zeta-x| \leqslant \omega(\zeta)\}, \\
& \omega^{-}(x)=\inf \{\omega(\zeta):|\zeta-x| \leqslant \omega(\zeta)\} .
\end{aligned}
$$

Легко проверить, что

$$
\rho_{B}^{+}(x) \leqslant \omega^{+}(x), \quad \rho_{E \mid B}^{-}(x) \geqslant \frac{\omega^{-}(x)}{2} .
$$

Подставляя в (2), получаем следуюшее утверждение. 
СлеДСТвиЕ. Пусть $\omega-$ положительная функиия, заданная на $\mathbb{R}^{m} ; E=\left\{e_{\alpha}\right\}$, $B=\left\{b_{\alpha}\right\}$ - системь шаров (5), (6). Предположим, что система Е имеет конечную кратность $k_{E}$. Тогда кратность $k_{B}(x)$ системь $B$ в точке $x$ допускает оценку

$$
k_{B}(x) \leqslant k_{E} \cdot 2^{2 m}\left(\frac{\omega^{+}(x)}{\omega^{-}(x)}\right)^{m} .
$$

\section{2. О разделяющих функциях.}

Пусть $g, G$ - непересекающиеся открытые множества в $\mathbb{R}^{m}$. Функция $\chi$, заданная на $\mathbb{R}^{m}$, разделяет g и $G$, если $0 \leqslant \chi(x) \leqslant 1 \forall x \in \mathbb{R}^{m}$ и

$$
\chi(x)= \begin{cases}0, & x \in g \\ 1, & x \in G .\end{cases}
$$

Цель этого пункта - построить функцию, разделяюшую $g$ и $G$ и допускаюшую определенную оценку для своего градиента.

Пусть $\omega$ - положительная непрерьвная ограниченная функция, заданная на $\mathbb{R}^{m}$. Рассмотрим функции $\omega^{+}, \omega^{-}$, определенные по формулам $(7),(8)$.

Лемма. Функиия $\omega^{+}(x)$ полунепрерывна сверху, $\omega^{-}(x)$ - положительна и полунепрерывна снизу.

Доказательство использует стандартные приемы математического анализа.

ПРЕДЛОЖЕНИЕ 8.2. Пусть для каждой точки $x \in G$ выполняется неравенство

$$
\omega(x) \leqslant \operatorname{dist}(x, g) .
$$

Тогда существует бесконечно дифференцируемая функция $\chi$, разделяющая $g$ и $G$, для градиента которой справедлива оценка

$$
|\operatorname{grad} \chi|(x) \leqslant \operatorname{const} \frac{1}{\omega^{-}(x)}\left(\frac{\omega^{+}(x)}{\omega^{-}(x)}\right)^{m},
$$

где константа зависит только от $\mathrm{m}$.

ДокАЗАТЕЛЬСТво. Введем обозначения

$$
\begin{aligned}
& e(x)=\left\{\zeta:|\zeta-x| \leqslant \frac{\omega(x)}{2}\right\}, \\
& b(x)=\{\zeta:|\zeta-x| \leqslant \omega(x)\} .
\end{aligned}
$$

По известной лемме о покрытии шарами [59; гл. III, §4] существует последовательность шаров $E=\left\{e\left(x_{n}\right)\right\}, x_{n} \in G$, покрывающая $G$ и имеющая конечную кратность $k_{E}(x) \leqslant$ const, где констанста зависит только от $m$. Рассмотрим соответствуюшую систему шаров $B=\left\{b\left(x_{n}\right)\right\}$. По следствию к предложению 8.1 кратность $k_{B}(x)$ системы $B$ в точке $x$ допускает оценку (9). По лемме $\omega^{+}$полунепрерьвна сверху; по той же лемме $\omega^{-}$полунепрерьвна снизу и положительна. Поэтому отношение $\omega^{+} / \omega^{-}$полунепрерьвно сверху. Отсюда, в частности, следует, что отношение $\omega^{+} / \omega^{-}$ограничено на любом компакте $K \subset G$. Из неравенства (9) следует, что $\sup _{x \in K} k_{B}(x)<\infty$. Это 
влечет, что каждый компакт $K \subset G$ может пересекаться лишш с конечным числом шаров из $B$. Действительно, в противном случае из бесконечного множества шаров из $B$, пересекаюшихся с $K$, можно извлечь последовательность шаров $b\left(x_{n}\right)$, сходящуюся к некоторому шару положительного радиуса с центром в некоторой точке $x_{0}$; при этом радиусы шаров $b\left(x_{n}\right)$ ограничены снизу положительным числом, paвным inf $K \omega$. Ясно, что точка $x_{0}$ содержится в бесконечном множестве шаров из $B$, что невозможно. Пусть $\sigma$ - положительная бесконечно дифференцируемая функция на $\mathbb{R}^{m}$, удовлетворяюшая условию $\sigma(x)=0$ при $|x| \geqslant 1$ и $\sigma(x) \geqslant 1$ при $|x| \leqslant 1 / 2$. Рассмотрим функции

$$
\chi_{n}(x)=\sigma\left(\frac{x-x_{n}}{\omega\left(x_{n}\right)}\right), \quad n=1,2, \ldots
$$

$\chi_{n}$ - финитная неотрицательная бесконечно дифференцируемая функция с носителем в $b\left(x_{n}\right)$. В шаре $e\left(x_{n}\right)$ функция $\chi_{n}$ принимает значения, большие или равные 1 . Рассмотрим ряд

$$
\chi=\sum \chi_{n}
$$

Ряд сходится по той причине, что каждый компакт пересекается с конечным числом носителей $b\left(x_{n}\right)$ функций $\chi_{n}$. По той же причине ряд можно дифференцировать почленно; значит, $\chi$ - бесконечно дифференцируемая функция. Кроме того, $\chi$ неотрицательна. При $x \in G$ сушествует $e\left(x_{n}\right), x \in e\left(x_{n}\right)$, и потому $\chi(x) \geqslant \chi_{n}(x) \geqslant 1$. Таким образом, $\chi \geqslant 1$ на $G$. В силу условия (10) носители $\chi_{n}$ не пересекаются с $g$. Поэтому $\chi=0$ на $g$.

Оценим $\left|\frac{\partial \chi}{\partial x_{i}}\right|$ в точке $x$. Поскольку носитель $\frac{\partial \chi_{n}}{\partial x_{i}}$ лежит в $b\left(x_{n}\right)$, имеем

$$
\left|\frac{\partial \chi}{\partial x_{i}}\right|(x) \leqslant \sum_{n}\left|\frac{\partial \chi_{n}}{\partial x_{i}}\right|(x)=\sum_{x \in b\left(x_{n}\right)}\left|\frac{\partial \chi_{n}}{\partial x_{i}}\right|(x) .
$$

Из выражения (12) для $\chi_{n}$ следует $\frac{\partial \chi_{n}}{\partial x_{i}}=\frac{\partial \sigma}{\partial x_{i}} \frac{1}{\omega\left(x_{n}\right)}$, откуда

$$
\left|\frac{\partial \chi_{n}}{\partial x_{i}}\right| \leqslant \frac{l}{\omega\left(x_{n}\right)}, \quad \text { где } \quad l=\max _{i} \sup _{\mathbb{R}^{m}}\left|\frac{\partial \sigma}{\partial x_{i}}\right| .
$$

Продолжая оценку, получим

$$
\left|\frac{\partial \chi}{\partial x_{i}}\right|(x) \leqslant l \sum_{x \in b\left(x_{n}\right)} \frac{1}{\omega\left(x_{n}\right)} .
$$

Далее, $\omega\left(x_{n}\right) \geqslant \omega^{-}(x)$ при $x \in b\left(x_{n}\right)$. Поэтому

$$
\left|\frac{\partial \chi}{\partial x_{i}}\right|(x) \leqslant \frac{l}{\omega^{-}(x)} \sum_{x \in b\left(x_{n}\right)} 1=l k_{B}(x) \frac{1}{\omega^{-}(x)} .
$$

Используя оценку (9) для $k_{B}(x)$, получаем

$$
\left|\frac{\partial \chi}{\partial x_{i}}\right|(x) \leqslant l k_{E} 2^{2 m}\left(\frac{\omega^{+}(x)}{\omega^{-}(x)}\right)^{m} \frac{1}{\omega^{-}(x)} .
$$


Отсюда следует оценка (11). Однако функция $\chi$ может быть не равной 1 на $G$. Мы подправим эту функцию следуюшим образом. Пусть $\varphi(t)-$ бесконечно дифференцируемая неубывающая функция на $\mathbb{R}$, удовлетворяющая условию: $\varphi(t)=0$ при $t \leqslant 0$ и $\varphi(t)=1$ при $t \geqslant 1$. Рассмотрим функцию $\widehat{\chi}(x)=\varphi(\chi(x))$. Эта функция принимает значения из $[0,1]$, бесконечно дифференщируема и $\widehat{\chi}=0$ на $g$ (ибо $\chi=0$ на $g$ ), $\widehat{\chi}=1$ на $G$ (ибо $\chi \geqslant 1$ на $G$ ). Далее, $\frac{\partial \hat{\chi}}{\partial x_{i}}=\varphi^{\prime} \cdot \frac{\partial \chi}{\partial x_{i}}$, откуда $\left|\frac{\partial \hat{\chi}}{\partial x_{i}}\right| \leqslant L\left|\frac{\partial \chi}{\partial x_{i}}\right|$, где $L=\max \left|\varphi^{\prime}\right|$. Используя последнее неравенство и (13), имеем

$$
\left|\frac{\partial \widehat{\chi}}{\partial x_{i}}\right| \leqslant L\left|\frac{\partial \chi}{\partial x_{i}}\right| \leqslant(L l) 2^{2 m} k_{E}\left(\frac{1}{\omega^{-}(x)}\right) \cdot\left(\frac{\omega^{+}(x)}{\omega^{-}(x)}\right)^{m} .
$$

Для завершения доказательства остается заметить, что оценка вида (11) для $\widehat{\chi}$ следует из (14) и неравенства

$$
|\operatorname{grad} \hat{\chi}| \leqslant m \max _{i}\left|\frac{\partial \widehat{\chi}}{\partial x_{i}}\right| .
$$

Функция $\sigma$, заданная на $\mathbb{R}^{m}$, имеет ограниченное колебание, если для любого $l>0$

$$
\sup _{\left|x_{1}-x_{2}\right| \leqslant l}\left|\sigma\left(x_{1}\right)-\sigma\left(x_{2}\right)\right|<\infty .
$$

СлЕДСТВИЕ. Пусть выполнено условие: для любого $x \in G$

$$
\ln \operatorname{dist}(x, g) \geqslant-\sigma(x),
$$

где $\sigma(x)$ - положительная непрерывная функция ограниченного колебания.

Тогда существует бесконечно дифферениируемая функиия $\chi$, разделяющая $g$ u $G$ и такая, что

$$
|\operatorname{grad} \chi|(x) \leqslant \operatorname{const} e^{\sigma(x)},
$$

где константа зависит только от $\mathrm{m}$.

ДоКАЗАТЕЛЬСТВо. Положим $\omega(x)=\exp (-\sigma(x)), \omega_{0}=\sup _{x} \omega(x), \omega^{+}(x)=$ $\sup _{\zeta}\{\omega(\zeta):|x-\zeta| \leqslant \omega(\zeta)\}, \omega^{-}(x)=\inf _{\zeta}\{\omega(\zeta):|x-\zeta| \leqslant \omega(\zeta)\}$. Оценим $\omega^{+}(x)$. Если $|x-\zeta| \leqslant \omega(\zeta)$, то $|x-\zeta| \leqslant \omega_{0}$. Следовательно,

$$
\begin{aligned}
\omega^{+}(x) & \leqslant \sup _{\zeta}\left\{\omega(\zeta):|x-\zeta| \leqslant \omega_{0}\right\} \\
& \leqslant \omega(x) \exp \sup _{\zeta}\left\{(\sigma(x)-\sigma(\zeta)):|x-\zeta| \leqslant \omega_{0}\right\}=\operatorname{const} \omega(x) .
\end{aligned}
$$

Аналогично,

$$
\begin{aligned}
\omega^{-}(x) & \geqslant \inf _{\zeta}\left\{\omega(\zeta):|x-\zeta| \leqslant \omega_{0}\right\} \\
& \geqslant \omega(x) \exp \inf _{\zeta}\left\{(\sigma(x)-\sigma(\zeta)):|x-\zeta| \leqslant \omega_{0}\right\}=\operatorname{const} \omega(x),
\end{aligned}
$$

где константы положительны. Таким образом,

$$
\omega^{+}(x) / \omega^{-}(x) \leqslant \text { const }, \quad \omega^{-}(x) \geqslant \text { const } \omega(x) .
$$

Согласно предложению 8.2 сушествует бесконечно дифференцируемая функция $\chi$, градиент которой удовлетворяет неравенству (11). Используя полученные выше оценки для $\omega^{+}(x) / \omega^{-}(x)$ и $\omega^{-}(x)$, получаем требуемое неравенство $(15)$. 


\section{$\S$ 9. Существование проекторов Дирихле в случае выпуклых $q$-областей}

1. Пусть $\Omega$ - вьпуклая $q$-область; $W$ - инвариантное подпространство в $H(\Omega)$, допускающее спектральньй синтез; $I$ - аннуляторньй подмодуль подпространства $W$; $\Lambda$ - квазиспектральное множество подпространства $W$. Используя обозначение п. 1 $\S 6$, можем написать: $W=W(\Lambda, \Omega)$. Согласно предложению 6.2 возможность аналитического продолжения элементов из $W$ в более широкую $q$-область $\Omega^{\prime}$ эквивалентна сушествованию проектора Дирихле

$$
p: W(\Lambda, \Omega) \rightarrow W\left(\Lambda, \Omega^{\prime}\right) .
$$

В этом параграфе мы исследуем задачу сушествования обшего проектора Дирихле

$$
p: W(\Lambda, \Omega) \rightarrow W\left(\Lambda^{\prime}, \Omega^{\prime}\right)
$$

где $\Lambda^{\prime}$ - часть $\Lambda$, отделенная от $\Lambda^{\prime \prime}=\Lambda \backslash \Lambda^{\prime}$ замкнутым множеством $\Gamma$, вдоль которого вьполняется специальная оценка снизу, $\Omega^{\prime}$ - вьпуклая $q$-область, определенным образом зависящая от $\Omega$. Теорема 9.1 дает условия существования проектора Дирихле (1) в терминах оценок “снизу" для независимой системы $q$-функций экспоненциального типа $u^{(1)}, \ldots, u^{(q)}$, локально принадлежаших $I$ в точках $\lambda \in \Lambda$. Теорема 9.1 доказывается методом, восходящим к К. Кисельману [38] и используюшим оценки Л. Хёрмандера [36] для решений неоднородных уравнений Коши-Римана.

Теорема 9.2 представляет собою упрошенный вариант теоремы 9.1.

\section{2. Терминология и обозначения.}

Чтобы сформулировать главньй результат статьи, нам понадобятся специальные понятия.

$1^{\circ}$. Пусть $\Gamma$ - замкнутое множество в $\mathbb{C}^{n}$, дополнение $\Gamma^{(c)}=\mathbb{C}^{n} \backslash \Gamma$ которого состоит из не менее чем двух компонент связности. Для фиксированной компоненты связности $e \subset \Gamma^{(c)}$ обозначим $E_{e}=\Gamma^{(c)} \backslash e$ объединение всех компонент связности $\Gamma^{(c)}$, отличных от $e$. Определим на $\Gamma^{(c)}$ функцию $T_{\Gamma}(z)$, полагая $T_{\Gamma}(z)=\operatorname{dist}\left(z, E_{e}\right)$ при $z \in e$.

Множество Г разделяет два множества $\Lambda^{\prime}, \Lambda^{\prime \prime}$, если существует разбиение $\Gamma^{(c)}$ на два открытых множества $Z^{\prime}, Z^{\prime \prime}$ таких, что $\Lambda^{\prime} \subset Z^{\prime}, \Lambda^{\prime \prime} \subset Z^{\prime \prime}$.

Ясно, что каждое из множеств $Z^{\prime}, Z^{\prime \prime}$ представляет собою объединение некоторого семейства компонент связности $\Gamma^{(c)}$.

Из определения следует: если $Г$ разделяет $\Lambda^{\prime}, \Lambda^{\prime \prime}$, то $\operatorname{dist}\left(z^{\prime}, \Lambda^{\prime \prime}\right) \geqslant T_{\Gamma}\left(z^{\prime}\right)$ при $z^{\prime} \in \Lambda^{\prime}, \operatorname{dist}\left(z^{\prime \prime}, \Lambda^{\prime}\right) \geqslant T_{\Gamma}\left(z^{\prime \prime}\right)$ при $z^{\prime \prime} \in \Lambda^{\prime \prime}$.

Множество Г окружает множество $\Lambda$, если существует разбиение $\Gamma^{(c)}$ на два открытых непустых множества $Z^{\prime}, Z^{\prime \prime}$, одно из которых, скажем, $Z^{\prime}$, содержит $\Lambda$. В этом случае будем также говорить, что Г есть окружение $\Lambda$.

$2^{\circ}$. Опорной функцией $q$-компакта $D=\left(D_{1}, \ldots, D_{q}\right)$ называется $q$-функция

$$
H_{D}(\lambda)=\left(H_{D_{1}}(\lambda), \ldots, H_{D_{q}}(\lambda)\right)
$$

где $H_{D_{i}}(\lambda)$ - опорная функция компакта $D_{i}$ в смысле комплексного анализа.

Понятие определяющего компакта целой функции экспоненциального типа было введено в $\S 1$, п. 4 . Пусть $u=\left(u_{1}, \ldots, u_{q}\right)$ - целая $q$-функция экспоненциального типа (т.е. все компоненты - целые функции экспоненциального типа). Выпукльй $q$-компакт 
$D=\left(D_{1}, \ldots, D_{q}\right)$ называется определяющим для q-функиии $u$, если $D_{i}-$ определяюший компакт для $u_{i}, i=1, \ldots, q$. То, что $D$ - определяюший $q$-компакт для $u$, равносильно следующему: для любого $\varepsilon>0$ сушествует константа $C$ такая, что выполняется неравенство

$$
|u(\lambda)| \leqslant C \exp \left(H_{D}(\lambda)+\varepsilon|\lambda|\right),
$$

где $H_{D}(\lambda)$ - опорная функция $q$-компакта $D$; при этом неравенство (2) следует трактовать как сокрашенную запись $q$ неравенств:

$$
\left|u_{i}(\lambda)\right| \leqslant C \exp \left(H_{D_{i}}(\lambda)+\varepsilon|\lambda|\right), \quad i=1, \ldots, q .
$$

Рассмотрим систему $u^{(1)}, \ldots, u^{(k)}$ из $k q$-функций экспоненциального типа. Вьпукльй $q$-компакт $D$ является определяющим для системы $u^{(1)}, \ldots, u^{(k)}$, если $D$ является определяюшим для каждой $q$-функшии $u^{(i)}, i=1, \ldots, k$.

\section{3. Формулировка теоремы для выпуклых $q$-областей.}

В этом пункте предполагается, что $\Omega$ - выпуклая $q$-область. Рассмотрим часть $\Lambda^{\prime}$ квазиспектрального множества $\Lambda$ инвариантного подпространства $W$. Будем предполагать, что замкнутое множество $\Gamma \subset \mathbb{C}^{n}$ разделяет $\Lambda^{\prime}$ и $\Lambda^{\prime \prime} ;$ если при этом $\Lambda^{\prime \prime}$ пусто, т.е. $\Lambda^{\prime}=\Lambda$, то Г окружает $\Lambda$ (п. 2). Пусть $I$ - аннуляторный подмодуль подпространства $W ; I(\lambda)$ - локальньй подмодуль (см. $\S 2$, п. 2 ), порождаемый $I$ в точке $\lambda$;

$$
u^{(1)}=\left(u_{1}^{(1)}, \ldots, u_{q}^{(1)}\right), \ldots, u^{(q)}=\left(u_{1}^{(q)}, \ldots, u_{q}^{(q)}\right)
$$

- независимая система $q$-функций экспоненщиального типа; $D$ - определяющий $q$-компакт системы $u^{(1)}, \ldots, u^{(q)} ; \Delta$ - определитель размера $q \times q$, состоящий из векторов-столбцов $u^{(1)}, \ldots, u^{(q)} ; \Delta_{i j}$ - алгебраическое дополнение элемента, стоящего на пересечении $i$-й строки и $j$-го столбца. В случае $q=1$ система (3) сводится к одной функции $u$, причем $\Delta_{i j}=1, \Delta=u$.

Напомним, что для множества $L \subset \mathbb{C}^{n}$ и множества направлений $\Theta \subset S^{2 n-1}$ $\operatorname{conv}_{\Theta} L$ обозначает $\Theta$-вьпуклую оболочку $L$ (п. $\left.4 \S 1\right)$. Если $L=\left(L_{1}, \ldots, L_{q}\right)-q$-множество, то $\operatorname{conv}_{\Theta} L:=\left(\operatorname{conv}_{\Theta} L_{1}, \ldots, \operatorname{conv}_{\Theta} L_{q}\right)$. Для неограниченного множества $L \subset \mathbb{C}^{n} \Theta_{L}$ обозначает предельное множество направлений этого множества (п. $4 \S 4$ ); $\Theta_{L}^{*}=\left\{\tau \in S^{2 n-1}: \bar{\tau} \in \Theta_{L}\right\}$ - множество сопряженных направлений.

\section{ТеОРема 9.1. Пусть выполняются условия:}

a) $\ln T_{\Gamma}(z) \geqslant-\sigma(z), z \in \Gamma^{(c)}$, әде $\sigma(z)$ - положительная функция ограниченного колебания $(\S 8$, п. 2$)$;

б)

$$
u^{(1)}, \ldots, u^{(q)} \in I(\lambda) \quad \forall \lambda \in \Lambda ;
$$

в) для некоторого выпуклого q-компакта $d=\left(d_{1}, \ldots, d_{q}\right)$ и некоторого выпуклого компакта $\omega \subset \mathbb{C}^{n}$ выполняются оценки

$$
\ln \left|\frac{\Delta_{i j}(\lambda)}{\Delta(\lambda)}\right|+H_{d_{i}}(\lambda)+\sigma(\lambda) \leqslant H_{\omega}(\lambda)+o(|\lambda|),
$$

$\lambda \rightarrow \infty, \lambda \in \Gamma, i, j=1, \ldots, q$

г) выполняется включение

$$
D+\omega \subset \Omega .
$$


Тогда существует проектор Дирихле

$$
p: W(\Lambda, \Omega) \rightarrow W\left(\Lambda^{\prime}, \Omega^{\prime}\right)
$$

где $\Omega^{\prime}=\operatorname{conv}_{\Theta_{\Gamma}^{*}} \operatorname{Int} d \cap \operatorname{conv}_{\Theta_{Z^{\prime}}^{*}} \Omega, Z^{\prime}-$ облединение компонент связности $\Gamma^{(c)}$, пересекающихся с $\Lambda^{\prime}$.

ЗАмечАниЕ 1. Условие в) следует понимать в следующем смысле: для любого $\varepsilon>0$ сушествует константа $C$ такая, что всюду в $Г$ выполняются неравенства

$$
\ln \left|\frac{\Delta_{i j}(\lambda)}{\Delta(\lambda)}\right|+H_{d_{i}}(\lambda)+\sigma(\lambda) \leqslant H_{\omega}(\lambda)+\varepsilon|\lambda|+C
$$

$i, j=1, \ldots, q$. Отсюда, между прочим, следует, что функции $\Delta_{i j}(\lambda) / \Delta(\lambda)$ голоморфны в некоторой окрестности $Г$.

ЗАмечАНИЕ 2 . При $q=1$ система $u^{(1)}, \ldots, u^{(q)}$ сводится к одной функции $u$ экспоненщиального типа, удовлетворяющей условию

$$
u \in I(\lambda) \quad \forall \lambda \in \Lambda
$$

а неравенства (5) - к оценке снизу

$$
\ln |u(\lambda)| \geqslant H_{d}(\lambda)-H_{\omega}(\lambda)+\sigma(\lambda)+o(|\lambda|)
$$

$\lambda \rightarrow \infty, \lambda \in \Gamma$.

\section{4. Вспомогательные леммы.}

Лемма 1. Пусть $D$ - определяющий q-компакт системы $u^{(1)}, \ldots, u^{(q)}$. Если для әтой системы вдоль множества Г выполняются оценки (5), то

$$
d \subset \operatorname{conv}_{\Theta_{\Gamma}^{*}}(D+\omega)
$$

ДОКАЗАТЕЛЬСТвО. По условию для любого $\varepsilon>0$ существует константа $C>0$ такая, что неравенства

$$
\ln \left|\frac{\Delta_{i j}(\lambda)}{\Delta(\lambda)}\right|+H_{d_{i}}(\lambda)+\sigma(\lambda) \leqslant H_{\omega}(\lambda)+\varepsilon|\lambda|+C, \quad i, j=1, \ldots, q,
$$

вьполняются на всем Г. Тем более на том же множестве вьполняются неравенства

$$
\ln \left|\frac{\Delta_{i j}(\lambda)}{\Delta(\lambda)}\right|+H_{d_{i}}(\lambda) \leqslant H_{\omega}(\lambda)+\varepsilon|\lambda|+C, \quad i, j=1, \ldots, q .
$$

По известному правилу разложения определителя по строкам имеем

$$
\sum_{j=1}^{q} \frac{\Delta_{i j}(\lambda)}{\Delta(\lambda)} u_{i}^{(j)}(\lambda)=1, \quad i=1, \ldots, q
$$


Отсюда следует

$$
\frac{1}{q} \leqslant \max _{j}\left|\frac{\Delta_{i j}(\lambda)}{\Delta(\lambda)}\right| \max _{j}\left|u_{i}^{(j)}(\lambda)\right|
$$

Согласно свойству определяющего $q$-компакта существует константа $C_{\varepsilon}$ такая, что

$$
\left|u_{i}^{(j)}(\lambda)\right| \leqslant C_{\varepsilon} \exp \left(H_{D_{i}}(\lambda)+\varepsilon|\lambda|\right), \quad i, j=1, \ldots, q
$$

Используя (7), (9), продолжим неравенство (8):

$$
\frac{1}{q} \leqslant \text { const } \cdot \exp \left(-H_{d_{i}}(\lambda)+H_{\omega}(\lambda)+H_{D_{i}}(\lambda)+2 \varepsilon|\lambda|\right) .
$$

Перепишем это неравенство в виде

$$
H_{d_{i}}(\lambda) \leqslant H_{D_{i}+\omega}(\lambda)+2 \varepsilon|\lambda|+\text { const }, \quad \lambda \in \Gamma,
$$

$i=1, \ldots, q$. Пусть $\tau_{0} \in \Theta_{\Gamma}$. Существует последовательность точек $\lambda_{n} \in \Gamma, \lambda_{n} \rightarrow \infty$, такая, что $\tau_{n}=\lambda_{n} /\left|\lambda_{n}\right| \rightarrow \tau_{0}$. Используя неравенство (10), имеем

$$
\begin{aligned}
\left|\lambda_{n}\right| H_{d_{i}}\left(\tau_{0}\right)= & \left|\lambda_{n}\right|\left(H_{d_{i}}\left(\tau_{0}\right)-H_{d_{i}}\left(\tau_{n}\right)\right)+\left|\lambda_{n}\right| H_{d_{i}}\left(\tau_{n}\right) \\
\leqslant & \left|\lambda_{n}\right|\left(H_{d_{i}}\left(\tau_{0}\right)-H_{d_{i}}\left(\tau_{n}\right)\right)+H_{D_{i}+\omega}\left(\lambda_{n}\right)+2 \varepsilon\left|\lambda_{n}\right|+\text { const } \\
\leqslant & \left|\lambda_{n}\right|\left(H_{d_{i}}\left(\tau_{0}\right)-H_{d_{i}}\left(\tau_{n}\right)\right)+\left|\lambda_{n}\right|\left(H_{D_{i}+\omega}\left(\tau_{n}\right)-H_{D_{i}+\omega}\left(\tau_{0}\right)\right) \\
& +\left|\lambda_{n}\right| H_{D_{i}+\omega}\left(\tau_{0}\right)+2 \varepsilon\left|\lambda_{n}\right|+\text { const. }
\end{aligned}
$$

В силу равномерной непрерьвности $H_{d_{i}}(\lambda)$ и $H_{D_{i}+\omega}(\lambda)$ на сфере $S^{2 n-1}$

$$
H_{d_{i}}\left(\tau_{0}\right)-H_{d_{i}}\left(\tau_{n}\right) \rightarrow 0, \quad H_{D_{i}+\omega}\left(\tau_{n}\right)-H_{D_{i}+\omega}\left(\tau_{0}\right) \rightarrow 0
$$

при $n \rightarrow \infty$. Поделим крайние члены в цепочке неравенств (11) на $\lambda_{n}$ и устремим $n$ к бесконечности. Совершая предельньй переход, получим

$$
H_{d_{i}}\left(\tau_{0}\right) \leqslant H_{D_{i}+\omega}\left(\tau_{0}\right)+2 \varepsilon .
$$

Это неравенство справедливо при любом $i=1, \ldots, q$, любом $\varepsilon>0$ и любом $\tau_{0} \in \Theta_{\Gamma}$. Следовательно,

$$
H_{d_{i}}(\tau) \leqslant H_{D_{i}+\omega}(\tau) \quad \forall \tau \in \Theta_{\Gamma} .
$$

В терминах опорных функций вешественного анализа эти неравенства записьваются в виде

$$
H_{d_{i}}^{(r)}(\tau) \leqslant H_{D_{i}+\omega}^{(r)}(\tau) \quad \forall \tau \in \Theta_{\Gamma}^{*}, \quad i=1, \ldots, q,
$$

или, что то же самое, в виде

$$
H_{d}^{(r)}(\tau) \leqslant H_{D+\omega}^{(r)}(\tau) \quad \forall \tau \in \Theta_{\Gamma}^{*} .
$$

В геометрических терминах это равносильно включению

$$
\operatorname{conv}_{\Theta_{\Gamma}^{*}} d \subset \operatorname{conv}_{\Theta_{\Gamma}^{*}}(D+\omega) .
$$

Тем более будет выполняться включение

$$
d \subset \operatorname{conv}_{\Theta_{\Gamma}^{*}}(D+\omega) .
$$


Лемма 2. Пусть $\Omega$ - выпуклая $q$-область, $M$ - неограниченное множсество в $\mathbb{C}^{n}$ смножеством предельных направлений $\Theta_{M}$. Тогда для любого q-компакта

$$
K^{\prime} \subset \operatorname{conv}_{\Theta_{M}^{*}} \Omega
$$

существуют q-компакт $K \subset \Omega$ иконстанты $\varepsilon>0, c<\infty$ такие, что

$$
H_{K^{\prime}}(z) \leqslant H_{K}(z)-\varepsilon|z|+c
$$

при $z \in M ;$ здесь $H_{K^{\prime}}, H_{K}$ - опорнье функиии q-компактов $K^{\prime}, K$ в смысле комплексного анализа.

ДокАЗАТЕЛЬСтво. Пусть $\Omega=\left(\Omega_{1}, \ldots, \Omega_{q}\right), K^{\prime}=\left(K_{1}^{\prime}, \ldots, K_{q}^{\prime}\right), M^{*}=\left\{z \in \mathbb{C}^{n}:\right.$ $\bar{z} \in M\}$. Очевидно, множество предельных направлений $M^{*}$ совпадает с $\Theta_{M}^{*}$. Включение (12) означает, что

$$
K_{i}^{\prime} \subset \operatorname{conv}_{\Theta_{M}^{*}} \Omega_{i}, \quad i=1, \ldots, q
$$

Согласно предложению 4.5 сушествуют компакты $K_{i} \subset \Omega_{i}$ и константы $\varepsilon_{i}, c_{i}>0$ такие, что

$$
H_{K_{i}^{\prime}}^{(r)}(z) \leqslant H_{K_{i}}^{(r)}(z)-\varepsilon_{i}|z|+c_{i} \quad \forall z \in M^{*}
$$

где $H_{K_{i}^{\prime}}^{(r)}, H_{K_{i}}^{(r)}$ - опорные функции компактов $K_{i}^{\prime}, K_{i}$ в смысле вешественного анализа. В терминах опорных функций комплексного анализа неравенства (14) переписываются в виде

$$
H_{K_{i}^{\prime}}(z) \leqslant H_{K_{i}}(z)-\varepsilon_{i}|z|+c_{i} \quad \forall z \in M, \quad i=1, \ldots, q .
$$

Эти неравенства будут вьполняться тем более, если $\varepsilon_{i}$ заменить на $\varepsilon=\min \varepsilon_{i}$, а $c_{i}-$ на $c=\max c_{i}$. Остается отметить, что неравенство (13) есть сокрашенная запись последних $q$ неравенств.

\section{5. Доказательство теоремы 9.1.}

Eсли Int $d=\varnothing$, то в соответствии с соглашением п. $1 \S 6$ проектор Дирихле (6) сушествует; поэтому в дальнейшем будем предполагать, что Int $d \neq \varnothing$. Дальнейшие рассуждения разобьем на 6 пунктов.

$1^{\circ}$. Пусть $I, I^{\prime}$ - аннуляторные подмодули подпространств $W, W^{\prime}$ соответственно. Согласно предложению 7.1 нужно построить непрерьвный мономорфизм Дирихле

$$
q: P^{\prime} / I^{\prime} \rightarrow P / I
$$

Для этого согласно предложению 7.2 достаточно для любого вьпуклого $q$-компакта $K^{\prime} \subset \Omega^{\prime}$ указать вьпуклый $q$-компакт $K$ со следуюшим свойством: для любой $q$-функщии $f^{\prime} \in P^{\prime}$ существует $q$-функция $f \in P$, удовлетворяющая условиям:

1) $f \equiv f^{\prime}(\bmod I(\lambda)) \forall \lambda \in \Lambda^{\prime}, f \equiv 0(\bmod I(\lambda)) \forall \lambda \in \Lambda \backslash \Lambda^{\prime}$.

2) $\|f\|_{K} \leqslant$ const $\left\|f^{\prime}\right\|_{K^{\prime}}$, где константа не зависит от $f, f^{\prime}$; 
$2^{\circ}$. Фиксируем выпукльй $q$-компакт $K^{\prime} \subset \Omega^{\prime}$. Ясно, что

$$
\begin{gathered}
K^{\prime} \subset \operatorname{conv}_{\Theta_{Z^{\prime}}^{*}} \Omega, \\
K^{\prime} \subset \operatorname{conv}_{\Theta_{\Gamma}^{*}} \operatorname{Int} d .
\end{gathered}
$$

По лемме 1

$$
d \subset \operatorname{conv}_{\Theta_{\Gamma}^{*}}(D+\omega) .
$$

Отсюда, используя условие г) теоремы, имеем

$$
\text { Int } d \subset d \subset \operatorname{conv}_{\Theta_{\Gamma}^{*}}(D+\omega) \subset \operatorname{conv}_{\Theta_{\Gamma}^{*}} \Omega \text {. }
$$

Значит, $\operatorname{conv}_{\Theta_{\Gamma}^{*}} \operatorname{Int} d \subset \operatorname{conv}_{\Theta_{\Gamma}^{*}} \Omega$. Следовательно,

$$
K^{\prime} \subset \operatorname{conv}_{\Theta_{\Gamma}^{*}} \Omega .
$$

Применяя лемму 2 к включениям (15), (16), заключаем, что существуют $q$-компакты $K_{1}, K_{2} \subset \Omega$ такие, что

$$
\begin{aligned}
& H_{K^{\prime}}(z) \leqslant H_{K_{1}}(z)-\varepsilon_{1}|z|+C_{1} \quad \forall z \in Z^{\prime}, \\
& H_{K^{\prime}}(z) \leqslant H_{K_{2}}(z)-\varepsilon_{2}|z|+C_{2} \quad \forall z \in \Gamma .
\end{aligned}
$$

Пусть $K$ - вьпукльй $q$-компакт в $\Omega$, содержаший одновременно $K_{1}$ и $K_{2}$. Тогда $H_{K_{1}}(z), H_{K_{2}}(z) \leqslant H_{K}(z)$ и неравенства $(17),(18)$ дают

$$
H_{K^{\prime}}(z) \leqslant H_{K}(z)-\varepsilon|z|+C, \quad z \in Z^{\prime} \cup \Gamma
$$

где $\varepsilon=\min \left[\varepsilon_{1}, \varepsilon_{2}\right], C=\max \left[C_{1}, C_{2}\right]$. Заметим, что, поскольку $D+\omega$ компактно вложено в $\Omega, q$-компакт $K$ можно выбрать таким образом, что

$$
D+\omega \Subset K
$$

Итак, по $q$-компакту $K^{\prime} \subset \Omega^{\prime}$ можем подобрать $q$-компакт $K \subset \Omega$ так, что вьполняются условия (19), (20).

$3^{\circ}$. Замкнутое множество Г разделяет $\Lambda^{\prime}$ и $\Lambda^{\prime \prime}$, т.е. $\Lambda^{\prime} \subset Z^{\prime}, \Lambda^{\prime \prime} \subset Z^{\prime \prime}$, где $Z^{\prime}, Z^{\prime \prime}-$ непересекаюшиеся открытые множества, составленные из компонент связности $\Gamma^{(c)}$. В качестве $Z^{\prime \prime}$ возьмем объединение $в c е x$ компонент связности $\Gamma^{(c)}$, не пересекающихся с $\Lambda^{\prime}$. Далее, по условию

$$
\ln T_{\Gamma}(z) \geqslant-\sigma(z), \quad z \in \Gamma^{(c)},
$$

где $\sigma(z)$ - положительная функшия ограниченного колебания. Из условия (21) следует (см. п. 1), что для любой точки $z \in Z^{\prime}$

$$
\ln \operatorname{dist}\left(z, Z^{\prime \prime}\right) \geqslant-\sigma(z)
$$


По следствию к предложению 8.2 сушествует положительная бесконечно дифференцируемая функция $\chi$, разделяющая $Z^{\prime \prime}$ и $Z^{\prime}$, т.е.

$$
\chi(z)=\left\{\begin{array}{l}
1, \quad z \in Z^{\prime}, \\
0, \quad z \in Z^{\prime \prime},
\end{array}\right.
$$

и такая, что

$$
|\operatorname{grad} \chi|(z) \leqslant \operatorname{const} e^{\sigma(z)}
$$

$4^{\circ}$. Фиксируем $q$-функцию $f^{\prime} \in P^{\prime}$. Наша цель - построить $q$-функцию $f$, которая удовлетворяет условиям 1), 2) п. $1^{\circ}$. Используя независимость системы $u^{(1)}, \ldots, u^{(q)}$ $\in I$, участвуюшей в условии б) теоремы, напишем представление

$$
f^{\prime}=\sum_{j=1}^{q} \frac{\Delta_{j}\left(f^{\prime}\right)}{\Delta} u^{(j)} .
$$

Здесь $\Delta$ - определитель, составленньй из векторов-столбцов $u^{(1)}, \ldots, u^{(q)} ; \Delta_{j}\left(f^{\prime}\right)-$ определитель, полученньй из $\Delta$ заменой $j$-го столбца столбцом $f^{\prime}$. Рассмотрим $q$-функцию

$$
\widehat{f}=\chi f^{\prime}=\sum_{j=1}^{q} l_{j} u^{(j)},
$$

где $l_{j}=\chi \frac{\Delta_{j}\left(f^{\prime}\right)}{\Delta}$. Ясно, что $q$-функция $\widehat{f}$ совпадает с $f^{\prime}$ на $Z^{\prime}$, равна нулю на $Z^{\prime \prime}$, и $|\widehat{f}| \leqslant\left|f^{\prime}\right|$ везде в $\mathbb{C}^{n} ;$ более того, $\widehat{f}$ является голоморфной на $Z^{\prime} \cup Z^{\prime \prime}$ и удовлетворяет условиям

$$
\widehat{f} \equiv f^{\prime} \quad(\bmod I(\lambda)), \quad \lambda \in \Lambda^{\prime} ; \quad \widehat{f} \equiv 0 \quad(\bmod I(\lambda)), \quad \lambda \in \Lambda^{\prime \prime} .
$$

Неравенство (19) влечет неравенство

$$
H_{K^{\prime}}(z) \leqslant H_{K}(z)+C, \quad z \in Z^{\prime} \cup \Gamma
$$

которое может быть расписано покомпонентно:

$$
H_{K_{i}^{\prime}}(z) \leqslant H_{K_{i}}(z)+C, \quad z \in Z^{\prime} \cup \Gamma, \quad i=1, \ldots, q .
$$

Пользуясь этими неравенствами и тем фактом, что $\widehat{f}=0$ вне $Z^{\prime} \cup \Gamma$, имеем

$$
\begin{aligned}
\|\widehat{f}\|_{K} & =\sum_{i=1}^{q} \sup _{\mathbb{C}^{n}} \frac{\left|\widehat{f}_{i}(z)\right|}{\exp H_{K_{i}}(z)} \leqslant \mathrm{const} \sum_{i=1}^{q} \sup _{Z^{\prime} \cup \Gamma} \frac{\left|f_{i}^{\prime}(z)\right|}{\exp H_{K_{i}^{\prime}}(z)} \\
& \leqslant \operatorname{const} \sum_{i=1}^{q} \sup _{\mathbb{C}^{n}} \frac{\left|f_{i}^{\prime}(z)\right|}{\exp H_{K_{i}^{\prime}}(z)}=\mathrm{const}\left\|f^{\prime}\right\|_{K^{\prime}} .
\end{aligned}
$$

Итак,

$$
\|\widehat{f}\|_{K} \leqslant \mathrm{const}\left\|f^{\prime}\right\|_{K^{\prime}},
$$

где констанста не зависит от $\widehat{f}, f^{\prime}$. 
$5^{\circ}$. Сравнения (24) и неравенство (25) означают, что $q$-функция $\widehat{f}$ удовлетворяет требованиям 1), 2) п. $1^{\circ}$. K сожалению, функция $\widehat{f}$ не является целой, ее аналитичность нарушается вдоль $Г$. Цель дальнейших рассуждений - подправить $\widehat{f}$ так, чтобы она стала целой и чтобы при этом сохранились условия $(24),(25)$. Будем действовать по следующему плану. Заменим коэффициенты $l_{j}$ в представлении (23) на разности $l_{j}-\widehat{l}_{j}$, где $\widehat{l}_{j}-$ бесконечно дифференщируемые функции, на которые наложены следующие требования:

1) $\widehat{l}_{j}$ голоморфны вне $\Gamma$;

2) разности $l_{j}-\widehat{l}_{j}-$ целые функции;

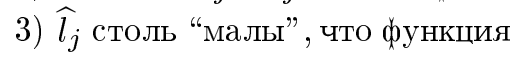

$$
f=\sum_{j=1}^{q}\left(l_{j}-\widehat{l}_{j}\right) u^{(j)}
$$

наследует неравенство (25).

В силу того, что $\widehat{l}_{j}$ голоморфны вне $\Gamma$, а $q$-функции $u^{(1)}, \ldots, u^{(q)}$ удовлетворяют условию (4), $q$-функция $f$ наследует также условие (24). Таким образом, задача сводится к построению функций $\widehat{l}_{j}$ с перечисленными свойствами. Мы это осушествим, беря в качестве $\widehat{l}_{j}$ решения с оценками уравнений Коши-Римана $\bar{\partial} \widehat{l}_{j}=\omega_{j}, j=1, \ldots, q$, где

$$
\omega_{j}=\sum_{i} \frac{\bar{\partial} l_{j}}{\partial \bar{z}_{i}} d \bar{z}_{i}
$$

- дифференциальная форма с коэффициентами

$$
\omega_{j i}=\frac{\bar{\partial} l_{j}}{\partial \bar{z}_{i}}=\frac{\Delta_{j}\left(f^{\prime}\right)}{\Delta} \cdot \frac{\bar{\partial}}{\partial \bar{z}_{i}} \chi .
$$

Из условия в) теоремы следует, что отношения $\Delta_{j}\left(f^{\prime}\right) / \Delta$ голоморфны в окрестности $\Gamma$; кроме того, $\frac{\bar{\partial} \chi}{\partial \bar{z}_{i}}-$ бесконечно дифференцируемые функции, обрашаюшиеся в нуль вне $\Gamma$. Поэтому $\omega_{j}-$ дифференциальные формы на $\mathbb{C}^{n}$ класса $C^{\infty}$, обращаюшиеся в нуль вне Г. Отметим также, что формы $\omega_{j}$ являются замкнутыми. Для дифференциальных форм $\omega_{j}=\sum \omega_{j i} d \bar{z}_{i}$ будем использовать стандартное обозначение

$$
\left|\omega_{j}\right|=\sqrt{\sum_{i=1}^{q}\left|\omega_{j i}\right|^{2}} .
$$

Оценим рост $\left|\omega_{j}\right|$ вдоль $Г$. Используя оценку (22) для $\operatorname{grad} \chi$, имеем

$$
\begin{aligned}
\left|\omega_{j}\right|(\lambda) & =\left|\frac{\Delta_{j}\left(f^{\prime}\right)(\lambda)}{\Delta(\lambda)}\right| \cdot|\bar{\partial} \chi(\lambda)| \leqslant\left|\frac{\Delta_{j}\left(f^{\prime}\right)(\lambda)}{\Delta(\lambda)}\right| \cdot|\operatorname{grad} \chi|(\lambda) \\
& \leqslant \text { const } \cdot \exp \sigma(\lambda) \cdot\left|\frac{\Delta_{j}\left(f^{\prime}\right)(\lambda)}{\Delta(\lambda)}\right|
\end{aligned}
$$


где константа зависит только от размерности и от $\sigma$. Далее,

$$
\left|\frac{\Delta_{j}\left(f^{\prime}\right)(\lambda)}{\Delta(\lambda)}\right| \leqslant \sum_{i=1}^{q}\left|\frac{\Delta_{i j}(\lambda)}{\Delta(\lambda)}\right| \cdot\left|f_{i}^{\prime}(\lambda)\right| .
$$

Из неравенств (27) и условия в) теоремы следует при $\lambda \rightarrow \infty, \lambda \in \Gamma$ :

$$
\begin{aligned}
\left|\omega_{j}\right|(\lambda) & \leqslant \operatorname{conste}^{\sigma(\lambda)+o(|\lambda|)} \sum_{i=1}^{q}\left|f_{i}^{\prime}(\lambda)\right| \exp \left(-H_{d_{i}}(\lambda)+H_{\omega}(\lambda)-\sigma(\lambda)\right) \\
& \leqslant e^{o(|\lambda|)} \sum_{i=1}^{q} \frac{\left|f_{i}^{\prime}(\lambda)\right|}{\exp H_{K_{i}^{\prime}}(\lambda)} \exp \left(-H_{d_{i}}(\lambda)+H_{\omega}(\lambda)+H_{K_{i}^{\prime}}(\lambda)\right) \\
& \leqslant\left\|f^{\prime}\right\|_{K^{\prime}} e^{o(|\lambda|)} \sum_{i=1}^{q} \exp \left(H_{K_{i}^{\prime}}(\lambda)+H_{\omega}(\lambda)-H_{d_{i}}(\lambda)\right) .
\end{aligned}
$$

Далее, $q$-компакт $K^{\prime}$ принадлежит $\operatorname{conv}_{\Theta_{\Gamma}^{*}} \operatorname{Int} d$. По лемме 2 существуют $q$-компакт $K_{0} \subset \operatorname{Int} d$ и $\varepsilon>0$ такие, что

$$
H_{K^{\prime}}(\lambda) \leqslant H_{K_{0}}(\lambda)-\varepsilon|\lambda|+\text { const }
$$

при $\lambda \in \Gamma$. Так как $K_{0} \subset \operatorname{Int} d$, то $H_{K_{0}}(\lambda) \leqslant H_{d}(\lambda) ;$ поэтому при $\lambda \in \Gamma$ имеем

$$
H_{K^{\prime}}(\lambda) \leqslant H_{d}(\lambda)-\varepsilon|\lambda|+\text { const. }
$$

Продолжая оценку (28), получаем

$$
\begin{aligned}
\left|\omega_{j}\right|(\lambda) & \leqslant \text { const }\left\|f^{\prime}\right\|_{K^{\prime}} \exp \left\{o(|\lambda|)+H_{\omega}(\lambda)-\varepsilon|\lambda|\right\} \\
& \leqslant \text { const }\left\|f^{\prime}\right\|_{K^{\prime}} \exp \left(H_{\omega}(\lambda)-(\varepsilon / 2)|\lambda|\right) .
\end{aligned}
$$

Эта оценка верна при $\lambda \in \Gamma$. Но $\omega_{j}=0$ вне $Г$. Поэтому оценка (29) верна на всем $\mathbb{C}^{n}$. Из этой оценки следует

$$
\int_{\mathbb{C}^{n}}\left|\omega_{j}\right|^{2}(\lambda) e^{-2 H_{\omega}(\lambda)} d s \leqslant \mathrm{const}\left\|f^{\prime}\right\|_{K^{\prime}}^{2}
$$

где $d s$ - элемент меры Лебега.

Согласно известной оценке Л. Хёрмандера [36; теорема 4.4.2] сушествует решение $\widehat{l}_{j}$ уравнения $\bar{\partial} \widehat{l}_{j}=\omega_{j}$ такое, что

$$
\int_{\mathbb{C}^{n}} \frac{\left|\widehat{l}_{j}(\lambda)\right|^{2} e^{-2 H_{\omega}(\lambda)}}{\left(1+|\lambda|^{2}\right)^{2}} d s \leqslant \frac{1}{2} \int_{\mathbb{C}^{n}}\left|\omega_{j}\right|^{2}(\lambda) e^{-2 H_{\omega}(\lambda)} d s .
$$

Продолжая это неравенство с помошью неравенства (30), получим

$$
\int_{\mathbb{C}^{n}} \frac{\left.\widehat{l}_{j}(\lambda)\right|^{2} e^{-2 H_{\omega}(\lambda)}}{\left(1+|\lambda|^{2}\right)^{2}} d s \leqslant \mathrm{const}\left\|f^{\prime}\right\|_{K^{\prime}}^{2} .
$$


В формуле (26) для $f$ мы будем брать в качестве $\widehat{l}_{j}$ эти решения.

$6^{\circ}$. Оценим $q$-функцию $f=\left(f_{1}, \ldots, f_{q}\right)$. Обозначим через $e(\lambda)$ шар с центром в $\lambda$ единичного радиуса. $|e(\lambda)|$ обозначает объем этого шара. В силу субгармоничности $\left|f_{i}\right|^{2}$ имеем

$$
\left|f_{i}(\lambda)\right| \leqslant \frac{1}{|e(\lambda)|^{1 / 2}}\left(\int_{e(\lambda)}\left|f_{i}\right|^{2} d s\right)^{1 / 2} .
$$

Компоненты $q$-функций $f=\left(f_{1}, \ldots, f_{q}\right), \widehat{f}=\left(\widehat{f_{1}}, \ldots, \widehat{f}_{q}\right), u^{(j)}=\left(u_{1}^{(j)}, \ldots, u_{q}^{(j)}\right)$ связаны соотношениями (см. (26))

$$
f_{i}=\widehat{f}_{i}-\sum_{j=1}^{q} \widehat{l}_{j} u_{i}^{(j)}, \quad i=1, \ldots, q .
$$

Используя неравенство Минковского, получаем

$$
\begin{aligned}
\left|f_{i}(\lambda)\right| & \leqslant \frac{1}{|e(\lambda)|^{1 / 2}}\left(\int_{e(\lambda)}\left|f_{i}\right|^{2} d s\right)^{1 / 2} \\
& \leqslant \frac{1}{|e(\lambda)|^{1 / 2}}\left(\int_{e(\lambda)}\left|\widehat{f}_{i}\right|^{2} d s\right)^{1 / 2}+\frac{1}{|e(\lambda)|^{1 / 2}} \sum_{j=1}^{q}\left(\int_{e(\lambda)}\left|\widehat{l}_{j} u_{i}^{(j)}\right|^{2} d s\right)^{1 / 2} \\
& =J_{i}^{0}(\lambda)+\sum_{j=1}^{q} J_{i}^{j}(\lambda) .
\end{aligned}
$$

Напомним, что $K_{i}$ обозначает $i$-ю компоненту $q$-компакта $K$. Имеем

$$
\begin{aligned}
J_{i}^{0}(\lambda) & =\frac{1}{|e(\lambda)|^{1 / 2}}\left(\int_{e(\lambda)}\left(|\widehat{f}(\zeta)| \exp \left(-H_{K_{i}}(\zeta)\right)\right)^{2} \exp \left(2 H_{K_{i}}(\zeta)\right) d s\right)^{1 / 2} \\
& \leqslant \exp \left(\max _{\zeta \in e(\lambda)} H_{K_{i}}(\zeta)\right) \cdot\left\|\widehat{f_{i}}\right\|_{K_{i}} \\
& \leqslant \operatorname{const} \exp \left(H_{K_{i}}(\lambda)\right)\|\widehat{f}\|_{K} \leqslant \text { const } \exp \left(H_{K_{i}}(\lambda)\right)\left\|f^{\prime}\right\|_{K^{\prime}} .
\end{aligned}
$$

В последних двух неравенствах мы воспользовались полуаддитивностью $H_{K_{i}}(\lambda)$ и неравенством (25). Оценим $J_{i}^{(j)}(\lambda)$. Имеем

$$
\begin{aligned}
\left|J_{i}^{(j)}(\lambda)\right| & =\frac{1}{|e(\lambda)|^{1 / 2}}\left(\int_{e(\lambda)}\left|\widehat{l}_{j}\right|^{2}\left|u_{i}^{(j)}\right|^{2} d s\right)^{1 / 2} \\
& =\frac{1}{|e(\lambda)|^{1 / 2}}\left(\int_{e(\lambda)} \frac{\left|\widehat{l}_{j}(\zeta)\right|^{2}}{\left(1+|\zeta|^{2}\right)^{2}}\left|u_{i}^{(j)}(\zeta)\right|^{2}\left(1+|\zeta|^{2}\right)^{2} d s\right)^{1 / 2} .
\end{aligned}
$$

В силу компактности вложения $D+\omega \Subset K\left(\right.$ см. п. $2^{\circ}$ ) сушествует $\delta>0$ такое, что

$$
H_{D+\omega}(\lambda) \leqslant H_{K}(\lambda)-\delta|\lambda|,
$$

а поскольку $D$ - определяюший $q$-компакт системы $u^{(1)}, \ldots, u^{(q)}$, справедлива оценка

$$
\left|u_{i}^{(j)}(\lambda)\left(1+|\lambda|^{2}\right)^{2}\right| \leqslant \text { const } \cdot \exp \left(H_{D_{i}}(\lambda)+\delta|\lambda|\right) .
$$


Используя равенства $(33)$ и полуаддитивность $H_{D_{i}}(\lambda), H_{\omega}(\lambda),|\lambda|$, оценим

$$
\begin{aligned}
\left|J_{i}^{(j)}(\lambda)\right| & \leqslant \text { const } \cdot \exp \left(H_{D_{i}}(\lambda)+\delta|\lambda|\right) \cdot\left(\int_{e(\lambda)} \frac{\left|\widehat{l}_{j}(\zeta)\right|^{2}}{\left(1+|\zeta|^{2}\right)^{2}} d s_{\zeta}\right)^{1 / 2} \\
& \leqslant \text { const } \cdot \exp \left(H_{D_{i}}(\lambda)+H_{\omega}(\lambda)+\delta|\lambda|\right) \cdot\left(\int_{e(\lambda)} \frac{\left|\widehat{l}_{j}(\zeta)\right|^{2} e^{-2 H_{\omega}(\zeta)}}{\left(1+|\zeta|^{2}\right)^{2}} d s_{\zeta}\right)^{1 / 2} .
\end{aligned}
$$

Заметим, что $H_{D_{i}}(\lambda)+H_{\omega}(\lambda)=H_{D_{i}+\omega}(\lambda)$. С помошью неравенств $(31),(34)$ получаем

$$
\left|J_{i}^{(j)}(\lambda)\right| \leqslant \text { const } \cdot \exp H_{K_{i}}(\lambda)\left\|f^{\prime}\right\|_{K^{\prime}} .
$$

Соединение неравенств (32), (35) дает

$$
\left|f_{i}(\lambda)\right| \leqslant \operatorname{const}\left\|f^{\prime}\right\|_{K^{\prime}} \exp H_{K_{i}}(\lambda) .
$$

Это неравенство справедливо при любом $i=1, \ldots, q$. Поэтому

$$
\|f\|_{K}=\sum_{i=1}^{q} \sup _{\lambda \in \mathbb{C}^{n}} \frac{\left|f_{i}(\lambda)\right|}{\exp H_{K_{i}}(\lambda)} \leqslant \mathrm{const}\left\|f^{\prime}\right\|_{K^{\prime}}
$$

где константа не зависит от $f, f^{\prime}$. Итак, цель, поставленная в п. $1^{\circ}$ доказательства, достигнута: для любого элемента $f^{\prime} \in P^{\prime}$ существует элемент $f \in P$, связанный с $f^{\prime}$ условиями 1$), 2)$ п. $1^{\circ}$.

Теорема доказана.

ТЕОРема 9.2. Пусть выполняются условия а), б) и в) теоремы 9.1 и, кроме того, выполняется условие

г)

$$
\text { Int } d \subset \operatorname{conv}_{\Theta_{Z^{\prime}}^{*}} \Omega .
$$

Тогда существует проектор Дирихле

$$
p: W(\Lambda, \Omega) \rightarrow W\left(\Lambda^{\prime}, \Omega^{\prime \prime}\right),
$$

əде $\Omega^{\prime \prime}=\operatorname{conv}_{\Theta_{\Gamma \cup Z^{\prime}}^{*}} \operatorname{Int} d$.

ДокАЗАТЕльСтво. Включение (36) влечет

$$
\operatorname{conv}_{\Theta_{Z^{\prime}}^{*}} \operatorname{Int} d \subset \operatorname{conv}_{\Theta_{Z^{\prime}}^{*}} \Omega \text {. }
$$

Используя это включение, имеем

$\Omega^{\prime \prime}=\operatorname{conv}_{\Theta_{\Gamma \cup Z^{\prime}}^{*}} \operatorname{Int} d=\operatorname{conv}_{\Theta_{\Gamma}^{*}} \operatorname{Int} d \cap \operatorname{conv}_{\Theta_{Z^{\prime}}^{*}}$ Int $d \subset \operatorname{conv}_{\Theta_{\Gamma}^{*}} \operatorname{Int} d \cap \operatorname{conv}_{\Theta_{Z^{\prime}}^{*}} \Omega=\Omega^{\prime}$.

Согласно предложению 6.8 существование проектора Дирихле (37) следует из существования проектора Дирихле (6). 


\section{§10. Существование проекторов Дирихле в случае невыпуклых $q$-областей}

1. Пусть $\Omega$ - открытое $q$-множество, $W$ - инвариантное подпространство в $H(\Omega)$, допускающее спектральный синтез, $I$ - аннуляторньй подмодуль $W$. Так как $W$ допускает спектральньй синтез, то $I$ - обильный подмодуль $(\S 2$, п. 2$) . I(\lambda)$ - локальньй подмодуль, порождаемый $I$ в точке $\lambda(\S 2$, п. 2).

Далее, пусть $\Lambda$ - квазиспектральное множество подпространства $W ; \Lambda^{\prime}-$ часть $\Lambda$; $\Lambda^{\prime \prime}=\Lambda \backslash \Lambda^{\prime} ; \Gamma$ - замкнутое множество, разделяющее $\Lambda^{\prime}, \Lambda^{\prime \prime} ; u^{(1)}, \ldots, u^{(q)}$ - независимая система $q$-функций экспоненциального типа; $D$ - определяюший $q$-компакт системы $u^{(1)}, \ldots, u^{(q)} ;$ определители $\Delta, \Delta_{i j}-$ те же, что и в условии в) теоремы 9.1.

Теорема 10.1. Пусть выполняются следующие условия:

1) $\ln T_{\Gamma}(z) \geqslant-\sigma(z), z \in \Gamma^{(c)}$, әде $\sigma(z)$ - положительная функиия ограниченного колебания;

2) имеют место включения

$$
u^{(1)}, \ldots, u^{(q)} \in I(\lambda) \quad \forall \lambda \in \Lambda ;
$$

3) для некоторого выпуклого q-компакта $d=\left(d_{1}, \ldots, d_{q}\right)$ и некоторого выпуклого компакта $\omega \subset \mathbb{C}^{n}$ выполняются оченки

$$
\ln \left|\frac{\Delta_{i j}(\lambda)}{\Delta(\lambda)}\right|+H_{d_{i}}(\lambda)+\sigma(\lambda) \leqslant H_{\omega}(\lambda)+o(|\lambda|),
$$

$$
i, j=1, \ldots, q \text {. }
$$

Тогда существует проектор Дирихле

$$
p: W(\Lambda, \Omega) \rightarrow W\left(\Lambda^{\prime}, \Omega^{\prime}\right),
$$

¿əe

$$
\begin{array}{lll}
\Omega^{\prime}=\operatorname{conv}_{(A, B)} \Omega, & e c \Omega u & \Lambda^{\prime}=\Lambda,
\end{array}
$$

3десь $A=D+\omega, B=\operatorname{conv}_{\Theta_{\Gamma}^{*}} d \cap \operatorname{conv}_{\Theta_{Z^{\prime}}^{*}}(D+\omega)$.

ДокАЗАТЕльство. $1^{\circ}$. Сначала докажем существование проектора Дирихле при дополнительных условиях

$$
\begin{gathered}
D+\omega \subset \Omega, \\
\operatorname{Int}(D+\omega) \neq \varnothing, \\
\operatorname{Int} d \neq \varnothing .
\end{gathered}
$$

Рассмотрим убываюшую последовательность выпуклых $q$-областей

$$
\Omega_{1} \supset \Omega_{2} \supset \cdots
$$


сходящуюся к $D+\omega$. Положим $H_{n}=H\left(\Omega_{n}\right) ; P_{n}=P\left(\Omega_{n}\right)$ - модуль, ассоциированный с $\Omega_{n} ; W_{n}=W\left(\Lambda, \Omega_{n}\right)$ - инвариантное подпространство в $H_{n}$, допускающее спектральный синтез с тем же квазиспектром (см. $\S 2$, п. 6) и квазиспектральным множеством, что и $W ; I_{n}$ - аннуляторный подмодуль подпространства $W_{n}$.

Так как $W_{n}$ допускает спектральньй синтез, то подмодуль $I_{n}$ является обильньм (в $P_{n}$ ); более того, по предложению $2.4 \Lambda$ является квазинулевым множеством этого подмодуля. Утверждаем, что при любом $n=1,2, \ldots q$-функции $u^{(1)}, \ldots, u^{(q)}$ удовлетворяют условию

$$
u^{(1)}, \ldots, u^{(q)} \in I_{n}(\lambda) \quad \forall \lambda \in \Lambda,
$$

где $I_{n}(\lambda)$ - локальньй подмодуль, порождаемьй $I_{n}$ в точке $\lambda$.

Действительно, по условию (1) $u^{(i)} \in I(\lambda), i=1, \ldots, q$; так как $W$ и $W_{n}$ имеют одинаковые квазиспектры со спектральньм множеством $\Lambda$, то по предложению 2.5 $I(\lambda)=I_{n}(\lambda) \forall \lambda \in \Lambda$; включения (9) следуют отсюда очевидным образом.

При любом $n=1,2, \ldots$ подпространство $W_{n}$ удовлетворяет условиям а)-г) теоремы 9.1. Действительно, условие 1) совпадает с условием а); система $u^{(1)}, \ldots, u^{(q)}$ удовлетворяет условию (9), совпадаюшему с условием б); условие 3 ) совпадает с условием в); включение (6) обеспечивает условие г). Применяя теорему 9.1 к подпространствам $W_{n}$, заключаем, что для каждого $n=1,2, \ldots$ сушествует проектор Дирихле

$$
p_{n}^{\prime}: W\left(\Lambda, \Omega_{n}\right) \rightarrow W\left(\Lambda^{\prime}, B_{n}\right),
$$

где $B_{n}=\operatorname{conv}_{\Theta_{\Gamma}^{*}} \operatorname{Int} d \cap \operatorname{conv}_{\Theta_{Z^{\prime}}^{*}} \Omega_{n}$. Ho $\operatorname{Int}(D+\omega) \subset \Omega_{n}$. По предложению 6.8 существуют проекторы Дирихле

$$
p_{n}: W\left(\Lambda, \Omega_{n}\right) \rightarrow W\left(\Lambda^{\prime}, B^{0}\right),
$$

где $B^{0}=\operatorname{conv}_{\Theta_{\Gamma}^{*}} \operatorname{Int} d \cap \operatorname{conv}_{\Theta_{Z^{\prime}}^{*}} \operatorname{Int}(D+\omega)$. По предложению 6.15 сушествование проекторов (10) влечет существование проектора Дирихле

$$
p: W(\Lambda, \Omega) \rightarrow W(\Lambda, \widehat{\Omega}),
$$

где $\widehat{\Omega}=\operatorname{conv}_{\left(A, B^{0}\right)} \Omega$, если $\Lambda^{\prime}=\Lambda$, и $\widehat{\Omega}=\operatorname{conv}_{\left(A, B^{0}\right)}\left((\Omega \ominus A)+B^{0}\right)$, если $\Lambda^{\prime} \neq \Lambda$. Положим

$$
\begin{array}{ll}
B_{1}^{0}=\operatorname{conv}_{\Theta_{\Gamma}^{*}} \operatorname{Int} d, & B_{1}=\operatorname{conv}_{\Theta_{\Gamma}^{*}} d \\
B_{2}^{0}=\operatorname{conv}_{\Theta_{Z^{\prime}}^{*}} \operatorname{Int}(D+\omega), & B_{2}=\operatorname{conv}_{\Theta_{Z^{\prime}}^{*}}(D+\omega) .
\end{array}
$$

По предложению 4.2 замыкания $\bar{B}_{1}^{0}, \bar{B}_{2}^{0}$ множеств $B_{1}^{0}, B_{2}^{0}$ совпадают с $B_{1}, B_{2}$ соответственно. Поэтому

$$
\bar{B}^{0}=\bar{B}_{1}^{0} \cap \bar{B}_{2}^{0}=B_{1} \cap B_{2}=B .
$$

По свойству 2 геометрической разности (см. §5, п. 1) $(\Omega \ominus A)+B^{0}=(\Omega \ominus A)+B$, а по свойству 8 нестандартной вьпуклости $\left(\S 5\right.$, п. 2) $\left(A, B^{0}\right)$-выпуклая оболочка любого открытого $q$-множества совпадает с его $(A, B)$-выпуклой оболочкой. Поэтому $q$-множество $\widehat{\Omega}$ совпадает с $q$-множеством $\Omega^{\prime}$, определенным по формулам (4), (5), а проектор (11) совпадает с проектором Дирихле (3). 
Итак, при дополнительных условиях (6)-(8) утверждение теоремы доказано.

$2^{\circ}$. Откажемся от условий $(7),(8)$ и докажем утверждение теоремыпри условии (6). Таким образом, $d$ и $D+\omega$ могут иметь пустые внутренности. Рассмотрим убьваюшую последовательность замкнутых шаров $b_{1} \supset b_{2} \supset \cdots$ с центром в начале координат и с положительньми радиусами, сходящимися к 0 . Полагаем $d_{n}=d+b_{n}, \omega_{n}=\omega+b_{n}$. Заметим, что Int $d_{n} \neq \varnothing, \operatorname{Int}\left(D+\omega_{n}\right) \neq \varnothing, n=1,2, \ldots$ Поскольку $D+\omega$ компактно принадлежит $\Omega$, радиусы шаров $b_{n}$ можно выбрать столь малыми, чтобы $D+\omega_{n} \subset \Omega$, $n=1,2, \ldots$. Итак, $\omega_{n}, d_{n}$ удовлетворяют условиям, аналогичным $(6)-(8)$ :

$$
D+\omega_{n} \subset \Omega, \quad \operatorname{Int}\left(D+\omega_{n}\right) \neq \varnothing, \quad \text { Int } d_{n} \neq \varnothing .
$$

Пусть $H_{b_{n}}(\lambda)$ - опорная функция $b_{n}$ в смысле комплексного анализа. Прибавим $H_{b_{n}}(\lambda)$ к обеим частям неравенств (2). Мы получим аналогичные неравенства, в которых $\omega$ заменено на $\omega_{n}$, а $d$ - на $d_{n}=d+\omega_{n}$. Из вьшеизложенного следует, что $D+\omega_{n}, d_{n}$ удовлетворяют условиям п. $1^{\circ}$, при которых утверждение теоремы уже доказано. Это позволяет заключить, что для каждого $n=1,2, \ldots$ существует проектор Дирихле

$$
\widehat{p}_{n}: W(\Lambda, \Omega) \rightarrow W\left(\Lambda^{\prime}, \widehat{\Omega}_{n}\right),
$$

где $\widehat{\Omega}_{n}=\operatorname{conv}_{\left(A_{n}, B_{n}\right)} \Omega$, если $\Lambda^{\prime}=\Lambda$, и

$$
\widehat{\Omega}_{n}=\operatorname{conv}_{\left(A_{n}, B_{n}\right)}\left(\left(\Omega \ominus A_{n}\right)+B_{n}\right),
$$

если $\Lambda^{\prime} \neq \Lambda$. Здесь $A_{n}=D+\omega_{n}, B_{n}=\operatorname{conv}_{\Theta_{\Gamma}^{*}} d_{n} \cap \operatorname{conv}_{\Theta_{Z^{\prime}}^{*}}\left(D+\omega_{n}\right)$.

Далее будем рассматривать случаи $\Lambda^{\prime}=\Lambda, \Lambda^{\prime} \neq \Lambda$ отдельно.

$3^{\circ}$. Случай $\Lambda^{\prime}=\Lambda$.

Напомним, что $A=D+\omega, B=\operatorname{conv}_{\Theta_{\Gamma}^{*}} d \cap \operatorname{conv}_{\Theta_{Z^{\prime}}^{*}}(D+\omega)$. Так как $d \subset d_{n}$, $\omega \subset \omega_{n}$, то $B \subset B_{n}$ и в силу свойства 5 нестандартной выпуклости $(\S 5$, п. 2$)$

$$
\operatorname{conv}_{\left(A_{n}, B\right)} \Omega \subset \operatorname{conv}_{\left(A_{n}, B_{n}\right)} \Omega .
$$

Поэтому по предложению 6.8 сушествование проектора Дирихле (12) влечет сушествование проектора Дирихле

$$
\widehat{\widehat{p}}_{n}: W(\Lambda, \Omega) \rightarrow W\left(\Lambda^{\prime}, \widehat{\widehat{\Omega}}_{n}\right),
$$

где $\widehat{\widehat{\Omega}}_{n}=\operatorname{conv}_{\left(A_{n}, B\right)} \Omega$, причем это верно при любом $n=1,2, \ldots$. Последовательность $q$-компактов $A_{n}, n=1,2, \ldots$, убывает и сходится к $A$. По предложению 5.3 последовательность $\widehat{\widehat{\Omega}}_{n}$, возрастая, сходится к $\operatorname{conv}_{(A, B)} \Omega$. В силу предложения 6.11 сушествование проекторов Дирихле (14) влечет существование проектора (3). В случае $\Lambda^{\prime}=\Lambda$ при наличии включения (6) теорема доказана.

$4^{\circ}$. Случай $\Lambda^{\prime} \neq \Lambda$.

Будем исходить из того, что существуют проекторы Дирихле $(12)$, где $\widehat{\Omega}_{n}$ определяются по формуле (13). Прежде всего, заметим, что $B \subset B_{n}$, следовательно, в силу свойства 5 нестандартной вьпуклости (см. $\S 5$, п. 2) имеем

$$
\operatorname{conv}_{\left(A_{n}, B\right)}\left(\left(\Omega \ominus A_{n}\right)+B\right) \subset \operatorname{conv}_{\left(A_{n}, B_{n}\right)}\left(\left(\Omega \ominus A_{n}\right)+B_{n}\right)=\widehat{\Omega}_{n} .
$$


По предложению 6.8 сушествование проектора Дирихле (12) влечет сушествование проектора Дирихле

$$
\widehat{\widehat{p}}_{n}:: W(\Lambda, \Omega) \rightarrow W\left(\Lambda^{\prime}, \widehat{\widehat{\Omega}}_{n}\right)
$$

где $\widehat{\widehat{\Omega}}_{n}=\operatorname{conv}_{\left(A_{n}, B\right)}\left(\left(\Omega \ominus A_{n}\right)+B\right)$. Это верно при любом $n=1,2, \ldots$ По свойству 1 геометрической разности $q$-множество $\left(\Omega \ominus A_{n}\right)+B$ является открытым. Открытым будет и $q$-множество $\widehat{\widehat{\Omega}}_{n}$ (см. следствие в п. $\left.3 \S 5\right)$. Далее, по свойству 3 геометрической разности $(\S 5$, п. 1$)$ возрастающая последовательность $q$-множеств $\left(\Omega \ominus A_{n}\right)+B$ сходится к $(\Omega \ominus A)+B$. По предложению 5.5 последовательность множеств $\widehat{\widehat{\Omega}}_{n}=\operatorname{conv}_{\left(A_{n}, B\right)}\left(\left(\Omega \ominus A_{n}\right)+B\right)$ сходится к $\Omega^{\prime}=\operatorname{conv}_{(A, B)}((\Omega \ominus A)+B)$.

По предложению 6.11 сушествование проекторов Дирихле (15) влечет сушествование проекторов Дирихле

$$
p: W(\Lambda, \Omega) \rightarrow W\left(\Lambda^{\prime}, \Omega^{\prime}\right)
$$

При условии (6) утверждение теоремы доказано.

$5^{\circ}$. Приступим к доказательству в общем случае.

Предположим, что $\Omega$ вообше не содержит сдвигов $A=D+\omega$. В этой ситуации $\operatorname{conv}_{(A, B)} \Omega=\Omega$ (см. $\S 5$, п. 2 , замечание 1$)$ и $\operatorname{conv}_{(A, B)}((\Omega \ominus A)+B)=\varnothing$, ибо $\Omega \ominus A=\varnothing$. В случае $\Lambda^{\prime}=\Lambda$ проектором Дирихле будет тождественное отображение пространства $W(\Lambda, \Omega)$ на себя; в случае $\Lambda^{\prime} \neq \Lambda$ проектор Дирихле сушествует по соглашению (см. $\S 6$, п. 1$)$.

Пусть $\Omega$ содержит некоторый сдвиг $A$, скажем, $A+c \subset \Omega$. Положим $D_{c}=D+c$, $d_{c}=d+c, A_{c}=D_{c}+\omega, B_{c}=\operatorname{conv}_{\Theta_{\Gamma}^{*}} d_{c} \cap \operatorname{conv}_{\Theta_{Z^{\prime}}^{*}}\left(D_{c}+\omega\right)$.

Включение $A+c \subset \Omega$ можно записать в виде

$$
D_{c}+\omega \subset \Omega .
$$

Рассмотрим систему

$$
u^{(1 c)}(\lambda)=u^{(1)}(\lambda) \exp \langle c, \lambda\rangle, \ldots, u^{(q c)}(\lambda)=u^{(q)}(\lambda) \exp \langle c, \lambda\rangle .
$$

Легко проверить, что $q$-компакт $D_{c}$ будет определяющим для системы (17). Точно так же, как по системе $u^{(1)}, \ldots, u^{(q)}$ составлялись определители $\Delta, \Delta_{i j}$, составим по системе (17) определители $\Delta^{(c)}, \Delta_{i j}^{(c)}$. Легко проверить, что условие 2) теоремы 10.1 можно записать в виде

$$
\ln \left|\frac{\Delta_{i j}^{(c)}(\lambda)}{\Delta^{(c)}(\lambda)}\right|+H_{d_{i c}}(\lambda)+\sigma(|\lambda|) \leqslant H_{\omega}(\lambda)+o(|\lambda|),
$$

$\lambda \rightarrow \infty, \lambda \in \Gamma, i, j=1, \ldots, q$. Здесь $d_{i c}-i$-я компонента $q$-компакта $d_{c}$. 
Соотношения $(16),(18)$ означают, что $q$-компакты $D_{c}, d_{c}$ удовлетворяют условиям, аналогичным тем, которым удовлетворяли $q$-компакты $D, d$ в пш. $2^{\circ}, 3^{\circ}$. Это позволяет заключить, что утверждение теоремы сохраняется при замене $D$ на $D_{c}$ и $d$ на $d_{c}$; именно, существует проектор Дирихле

$$
p_{c}: W(\Lambda, \Omega) \rightarrow W\left(\Lambda^{\prime}, \widehat{\Omega}_{c}\right),
$$

где $\widehat{\Omega}_{c}=\operatorname{conv}_{\left(A_{c}, B_{c}\right)} \Omega$, если $\Lambda^{\prime}=\Lambda$, и $\widehat{\Omega}_{c}=\operatorname{conv}_{\left(A_{c}, B_{c}\right)}\left(\left(\Omega \ominus A_{c}\right)+B_{c}\right)$, если $\Lambda^{\prime} \neq \Lambda$. Теперь заметим, что

$$
\begin{gathered}
\left(\Omega \ominus A_{c}\right)+B_{c}=(\Omega \ominus(A+c))+B+c=(\Omega \ominus A)+B ; \\
A_{c}=D_{c}+\omega=A+c ; \\
B_{c}=\operatorname{conv}_{\Theta_{\Gamma}^{*}} d_{c} \cap \operatorname{conv}_{\Theta_{Z^{\prime}}^{*}}\left(D_{c}+\omega\right)=\operatorname{conv}_{\Theta_{\Gamma}^{*}} d \cap \operatorname{conv}_{\Theta_{Z^{\prime}}^{*}}(D+\omega)+c=B+c .
\end{gathered}
$$

Отсюда следует, что $\left(A_{c}, B_{c}\right)$-вьпуклость эквивалентна $(A, B)$-вьпуклости. Из вышеизложенного следует, что $\widehat{\Omega}_{c}=\operatorname{conv}_{(A, B)} \Omega$, если $\Lambda^{\prime}=\Lambda$, и $\widehat{\Omega}_{c}=$ $\operatorname{conv}_{(A, B)}((\Omega \ominus A)+B)$, если $\Lambda^{\prime} \neq \Lambda$. Таким образом, проектор Дирихле (19) совпадает с проектором Дирихле (3), о котором идет речь в утверждении теоремы. Теорема доказана полностью.

2. Теорема 10.1 влечет многочисленные следствия, которые в своей совокупности могут составить предмет отдельного исследования. Мы ограничимся выводом из теоремы 10.1 ряда утверждений, ведуших к теореме об аналитическом продолжении, сформулированной в $\S 1$, п. 6 . Прежде всего, отметим, что из формулировки теоремы 10.1 можно устранить функцию $\sigma(z)$, точнее говоря, справедлив следуюший вариант теоремы 10.1.

ТеОРема 10.2. Пусть выполняются следующие условия:

1)

$$
\lim _{\substack{|z| \rightarrow \infty \\ z \in \Gamma^{(c)}}} \frac{\ln T_{\Gamma}(z)}{|z|} \geqslant 0 ;
$$

2) $u^{(1)}, \ldots, u^{(q)} \in I(\lambda) \quad \forall \lambda \in \Lambda$;

3) для некоторого выпуклого q-компакта $d=\left(d_{1}, \ldots, d_{q}\right)$ и некоторого выпуклого компакта $\omega \subset \mathbb{C}^{n}$ вылполняются оценки

$$
\ln \left|\frac{\Delta_{i j}(\lambda)}{\Delta(\lambda)}\right|+H_{d_{i}}(\lambda) \leqslant H_{\omega}(\lambda)+o(|\lambda|),
$$

$\lambda \in \Gamma, \lambda \rightarrow \infty, i, j=1,2, \ldots$.

Тогда существует проектор Дирихле

$$
p: W(\Lambda, \Omega) \rightarrow W\left(\Lambda^{\prime}, \Omega^{\prime}\right),
$$

где $\Omega^{\prime}-(A, B)$-выпуклая оболочка $\Omega$, если $\Lambda^{\prime}=\Lambda$, и $\Omega^{\prime}-(A, B)$-выпуклая оболочка $(\Omega \ominus A)+B$, если $\Lambda^{\prime} \neq \Lambda$. 
ДоКАЗАТЕЛЬСТво. Положим

$$
\left(\ln T_{\Gamma}(z)\right)^{-}= \begin{cases}0, & \text { если } \ln T_{\Gamma}(z) \geqslant 0, \\ -\ln T_{\Gamma}(z), & \text { если } \ln T_{\Gamma}(z)<0 .\end{cases}
$$

Очевидно, что

$$
\ln T_{\Gamma}(z) \geqslant-\left(\ln T_{\Gamma}(z)\right)^{-}
$$

и в силу $(20)$

$$
\lim _{\substack{|z| \rightarrow \infty \\ z \in \Gamma^{(c)}}} \frac{\left(\ln T_{\Gamma}(z)\right)^{-}}{|z|}=0 .
$$

Пусть $\sigma(t)$ - вогнутая функция при $-\infty<t<+\infty$, удовлетворяющая условиям: $\sigma(t)=o(t)$ при $t \rightarrow \infty,\left(\ln T_{\Gamma}(z)\right)^{-} \leqslant \sigma(|z|), z \in \Gamma^{(c)}$. Такую функцию можно построить в силу соотношения (24). В силу $(23) \ln T_{\Gamma}(z) \geqslant-\sigma(|z|) ;$ кроме того, в силу вогнутости $\sigma(t)$ функция $\sigma(|z|)$ имеет ограниченное колебание. Это значит, что выполнено условие 1) теоремы 10.1 .

Добавим к обеим частям неравенств $(21)$ функцию $\sigma(|\lambda|)$. Тогда, используя соотношение $\sigma(|\lambda|)=o(|\lambda|)$, можно записать эти неравенства в виде неравенств (2). Значит, вьполнено условие 3) теоремы 10.1. Наконец, условие 2) теоремы 10.1 совпадает с условием 2) теоремы 10.2. Таким образом, все условия теоремы 10.1 выполнены. Согласно утверждению этой теоремы существует проектор Дирихле (22).

Рассмотрим случай $q=1$. Пусть $\Omega$ - открытое множество в $\mathbb{C}^{n}, W$ - инвариантное подпространство в $H(\Omega)$, допускаюшее спектральньй синтез, $\Lambda$ - квазиспектральное множество подпространства $W$, разделенное на части $\Lambda^{\prime}, \Lambda^{\prime \prime}$ замкнутьм множеством $\Gamma, I$ - аннуляторный подмодуль подпространства $W, I(\lambda)$ - локальный подмодуль, порождаемый $I$ в точке $\lambda$. Полагая в формулировке теоремы $10.2 q=1$, получим следующее утверждение.

ТеОРема 10.3. Пусть выполняются следующие условия:

1)

$$
\lim _{\substack{|z| \rightarrow \infty \\ z \in \Gamma^{(c)}}} \frac{\ln T_{\Gamma}(z)}{|z|} \geqslant 0 ;
$$

2) $u \in I(\lambda) \quad \forall \lambda \in \Lambda$;

3) $\ln |u(\lambda)|+H_{\omega}(\lambda) \geqslant H_{d}(\lambda)+o(|\lambda|), \lambda \rightarrow \infty, \lambda \in \Gamma$.

Тогда существует проектор Дирихле

$$
p: W(\Lambda, \Omega) \rightarrow W\left(\Lambda^{\prime}, \Omega^{\prime}\right),
$$

гдe

$$
\Omega^{\prime}=\operatorname{conv}_{(A, B)} \Omega,
$$

$e c \curvearrowright u \Lambda^{\prime}=\Lambda, u$

$$
\Omega^{\prime}=\operatorname{conv}_{(A, B)}((\Omega \ominus A)+B),
$$

если $\Lambda^{\prime} \neq \Lambda$. Здесь $A=D+\omega, B=\operatorname{conv}_{\Theta_{\Gamma}^{*}} d \cap \operatorname{conv}_{\Theta_{Z^{\prime}}^{*}}(D+\omega)$. 
Положим в формулировке этой теоремы $d=d_{0}+\omega$. Тогда условие 3$)$ примет вид

$$
\ln |u(\lambda)| \geqslant H_{d_{0}}(\lambda)+o(|\lambda|),
$$

а в выражении для $B d$ заменится на $d_{0}+\omega$. Таким образом, переобозначая $d_{0}$ буквой $d$, получаем утверждение.

ТЕОРЕМА 10.4. Пусть выполняются условия:

1)

$$
\varliminf_{\substack{|z| \rightarrow \infty \\ z \in \Gamma^{(c)}}} \frac{\ln T_{\Gamma}(z)}{|z|} \geqslant 0
$$

2) $u \in I(\lambda) \quad \forall \lambda \in \Lambda$;

3) $\ln |u(\lambda)| \geqslant H_{d}(\lambda)+o(|\lambda|), \lambda \rightarrow \infty, \lambda \in \Gamma$.

Тогда для любого выпуклого компакта $\omega \subset \mathbb{C}^{n}$ существует проектор Дирихле (25), где $\Omega^{\prime}$ определяется по формулам (26), (27) и $A=D+\omega$, $B=\operatorname{conv}_{\Theta_{\Gamma}^{*}}(d+\omega) \cap \operatorname{conv}_{\Theta_{Z^{\prime}}^{*}}(D+\omega)$.

Применим эту теорему при $\Lambda^{\prime}=\Lambda$ и при условии, что замкнутое множество $\Gamma$ окружает $\Lambda$. Мы получим, что при выполнении условий 1)-3) теоремы 10.4 каждый элемент $f \in W$ допускает аналитическое продолжение в $(A, B)$-вьпуклую оболочку $\Omega$.

Положим $B^{\prime}=\operatorname{conv}_{\Theta_{\Gamma \cup Z^{\prime}}^{*}}(d+\omega)$. Предполагая, что

$$
d \subset D
$$

имеем

$$
B^{\prime}=\operatorname{conv}_{\Theta_{\Gamma}^{*}}(d+\omega) \cap \operatorname{conv}_{\Theta_{Z^{\prime}}^{*}}(d+\omega) \subset \operatorname{conv}_{\Theta_{\Gamma}^{*}}(d+\omega) \cap \operatorname{conv}_{\Theta_{Z^{\prime}}^{*}}(D+\omega)=B
$$

По свойству 5 нестандартной выпуклости $(\S 5$, п. 2)

$$
\operatorname{conv}_{\left(A, B^{\prime}\right)} \Omega \subset \operatorname{conv}_{(A, B)} \Omega
$$

и, следовательно, если элемент $f \in W$ допускает аналитическое продолжение в $\operatorname{conv}_{(A, B)} \Omega$, то он тем более допускает аналитическое продолжение в $\operatorname{conv}_{\left(A, B^{\prime}\right)} \Omega$.

Таким образом, при вьполнении условий 1)-3) теоремы 10.4 и дополнительном условии (28) каждьй элемент $f \in W$ допускает аналитическое продолжение в $\operatorname{conv}_{\left(A, B^{\prime}\right)} \Omega$. Этот факт составляет содержание теоремы об аналитическом продолжении, анонсированной в $§ 1$, п. 6 .

\section{СПИСОК ЛИТЕРАТУРЫ}

[1] L. Schwartz. Théorie générale des fonctions moyenne-périodique // Ann. of Math. (2). 1947. V. 48. № 4. P. 857-929.

[2] И.Ф. Красичков-Терновский. Инвариантные подпространства аналитических функций. I: Спектральный анализ на выпуклых областях // Матем. сб. 1972. Т. 87 (129). № 4. C. $459-489$. 
[3] И.Ф. Красичков- Терновский. Инвариантные подпространства аналитических функций. II: Спектральный синтез на выпуклых областях // Матем. сб. 1972. Т. 88 (130). №1. C. $3-30$.

[4] И.Ф. Красичков- Терновский. Инвариантные подпространства аналитических функций. III: О распространении спектрального синтеза // Матем. сб. 1972. Т. 88 (130). № 3. C. $331-352$.

[5] И.Ф. Красичков-Терновский. Спектральньй синтез аналитических функций на системах выпуклых областей // Матем. сб. 1980. Т. 111 (153). № 1. С. 3-41.

[6] И.Ф. Красичков-Терновский. Спектральный синтез аналитических функций на системах выпуклых областей. Распространение синтеза // Матем. сб. 1980. Т. 112 (154). № 1. C. $94-114$.

[7] И.Ф. Красичков- Терновский. Спектральньй синтез аналитических функций на системах неограниченных вьпуклых областей // Матем. сб. 1980. Т. 111 (153). № 3. С. 384-401.

[8] И.Ф. Красичков-Терновский. Для каждого инвариантного подпространства, допускающего спектральный синтез, существует метод аппроксимации // Сиб. матем. журн. 1981. T. 22 (154). № 3. C. 74-90.

[9] I. F. Krasičkov-Ternovskii. Spectral synthesis on a system of unbounded domains starlike in a common direction // Anal. Math. 1993. V. 19. № 3. P. 217-223.

[10] B. Malgrange. Existence et approximation des solutions des équations aux dérivées partielles et des équations de convolution // Ann. Inst. Fourier (Grenoble). 1955-1956. V. 6. P. 271-355.

[11] L. Ehrenpreis. Mean periodic functions. I: Varieties whose annihilator ideals are principal // Amer. J. Math. 1955. V. 77. P. 293-328.

[12] Р. С. Юлмухаметов. Однородные уравнения свертки // Докл. АН СССР. 1991. Т. 316. № 2. C. $312-315$.

[13] А. С. Кривошеев, В.В. Напалков. Комплексньй анализ и операторы свертки // УМН. 1992. Т. 47. №6. С. 3-58.

[14] А. Б. Шишкин. Спектральньй синтез для систем дифференциальных операторов с постоянными коэффициентами. Теорема двойственности // Матем. сб. 1998. Т. 189. №9. C. $143-160$.

[15] И.Ф. Красичков- Терновский. Спектральный синтез и локальное описание для многих переменных // Изв. РАН. Сер. матем. 1999. Т. 63. № 4. С. 101-130.

[16] В. В. Напалков. Уравнения свертки в многомерных пространствах. М.: Наука, 1982.

[17] А.С. Кривошеев. Аналитическое продолжение функций из инвариантных подпространств в выпуклых областях комплексного пространства // Изв. РАН. Сер. матем. 1998. T. 62. № 2. C. $77-102$.

[18] L. Ehrenpreis. Appendix to the paper: "Mean periodic functions I" // Amer. J. Math. 1955. V. 77. P. 731-733.

[19] J. Hadamard. Essai sur l'étude des fonctions donnés par leur développement de Taylor // J. Math. Pures Appl. Sér. (4). 1892. V. 4 (8). P. 101-106.

[20] E. Fabry. Sur les points singuliers d'une fonction donnée par son développement de Taylor // Ann. École Norm. Sup. (3). 1896. V. 13. P. 367-399.

[21] G. Pólya. Eine Verallgemeinerung des Fabryschen Lückensatzes // Nachr. Ges. Wiss. Göttingen, Math.-Phys. Kl. 1927. V. 2. P. 187-195.

[22] A. Ostrowski. Über die analytishe Fortsetzung von Taylorshen und Dirichletshen Reihen // Math. Ann. 1955. V. 129. P. 1-43.

[23] V. Bernstein. Leçons sur les progrès récents de la théorie des séries de Dirichlet. Paris: Gauthier-Villars, 1933.

[24] G. Pólya. Über die Existenz unendlich vieler singulärer Punkte auf der Konvergenzgeraden gewisser Dirichlet'sher Reihen // Sitzungber. Preuß. Akad. Wiss. 1923. P. 45-50.

[25] А.Ф. Леонтьев. О классе функций, определенных рядом полиномов Дирихле // УМН. 1948. T. 3. № 4. C. 3-58.

[26] А.Ф. Леонтьев. Ряды полиномов Дирихле и их обобщения // Труды МИАН. 1951. T. 39 . 
[27] J.-P. Kahane. Sur quelques problèmes d'unicité et de prolongement, relatifs aux fonctions approchables par des sommes d'exponentielles // Ann. Inst. Fourier (Grenoble). 1953-1954. V. 5. P. 39-130.

[28] А.Ф. Леонтьев. О сходимости последовательностей полиномов Дирихле // Докл. АН CCCP.. T. 108. № 1. C. 23-26.

[29] А. Ф. Леонтьев. Новое доказательство одной теоремы о сходимости последовательностей полиномов Дирихле // УМН. 1957. Т. 12. № 3. С. 165-170.

[30] А. Ф. Леонтьев. О свойствах последовательностей полиномов Дирихле, сходящихся на интервале мнимой оси // Изв. АН СССР. Сер. матем. 1965. Т. 29. № 2. С. 261-328.

[31] L. Schwartz. Étude des sommes d'exponentielles. Paris: Hermann, 1959.

[32] A. Baillette. Approximation de fonctions par des sommes d'exponentielles // C. R. Acad. Sci. Paris. 1959. V. 249. № 23. P. 2470-2471.

[33] A. Bailette. Fonctions approchables par des sommes d'exponentielles // J. Anal. Math. 1962-1963. V. 10. P. 91-115.

[34] И.Ф. Красичков. О сходимости полиномов Дирихле // Сиб. матем. журн. 1966. Т. 7. № 5. С. 1039-1058.

[35] И.Ф. Красичков- Терновский. Инвариантные подпространства аналитических функций. Аналитическое продолжение // Изв. АН СССР. Сер. матем. 1973. Т. 37. № 4. C. $931-945$.

[36] Л. Хёрмандер. Введение в теорию функций нескольких комплексных переменных. М.: Мир, 1968.

[37] P. Schapira. Équations aux dérivées partielles dans l'espace des hyperfonctions // Lecture Notes in Math. 1968. V. 71. P. 38-45.

[38] C. O. Kiselman. Prolongement des solutions d'une équation aux dérivées partielles à coefficients constants // Bull. Soc. Mat. France. 1969. V. 97. P. 329-356.

[39] L. Ehrenpreis. Fourier analysis in several complex variables. New York: Wiley, 1970. (Pure Appl. Math. V. 17.)

[40] В. П. Паламодов. Линейные дифференциальные операторы с постоянными коэффициентами. М.: Наука, 1967.

[41] A. Sebbar. Prolongement des solutions holomorphes de certains opérateurs différentiels d'ordre infini à coefficients constants // Lecture Notes in Math. 1980. V. 822. P. 199-220.

[42] A. Meril, D.S. Struppa. Convolutors in spaces of holomorphic functions // Lecture Notes in Math. 1987. V. 1276. P. 253-275.

[43] А. С. Кривошеев. Об индикаторе целшіх функций и продолжении решений однородного уравнения свертки // Матем. сб. 1993. Т. 184. № 8. С. 81-108.

[44] Ж. Себастьян-и-Сильва. О некоторых классах локально-выпуклых пространств, важных в приложениях // Математика. Сб. переводов. 1957. Т. 1. № 1. С. 60-77.

[45] Л.В. Канторович, Г.П. Акилов. Функционалшный анализ в нормированных пространствах. М.: Физматгиз, 1959.

[46] И.Ф. Красичков- Терновский. Локальное описание замкнутых идеалов и подмодулей аналитических функций одной переменной. I // Изв. АН СССР. Сер. матем. 1979. Т. 43. № 1. C. 44-66.

[47] H. Cartan. Idéaux et modules de fonctions analytiques de variables complexes // Bull. Soc. Math. France. 1950. V. 78. № 4. P. 29-64.

[48] ЖК. Дьедонне, Л. Шварц. Двойственность в пространствах $(F)$ и $(L F) / /$ Математика. Сб. переводов. 1958. Т. 2. № 2. С. 77-102.

[49] Р. Рокафеллар. Вьпукльй анализ. М.: Мир, 1973.

[50] В. Г. Болтянский. О некоторых классах выпуклых множеств // Докл. АН СССР. 1976. T. 226. № 1. С. 19-22.

[51] В. Г. Болтянский. Теория Хелли для $H$-выпуклых множеств // Докл. АН СССР. 1976. Т. 226. № 2. С. 249-252.

[52] Л. Данцер, Б. Грюнбаум, В. Кли. Теорема Хелли. М.: Мир, 1968.

[53] В. Г. Болтянский, П. С. Солтан. Комбинаторная геометрия различных классов выпуклых множеств. Кишинев: Штиинца, 1978. 
[54] В. Г. Болтянский, Э. Д. Баладзе. Проблема Секефальви-Надя в комбинаторной геометрии. М.: Наука, 1997.

[55] Р. Энгелькинг. Общая топология. М.: Мир, 1986.

[56] Л. С. Понтрягин. Линейные дифференциальные игры преследования // Матем. сб. 1980. T. 112. №3. C. 307-330.

[57] S. Banach. Théorie des opérations linéaires. Warszawa: Subwncji Funduszu Narodowej, 1932.

[58] А. Робертсон, В. Робертсон. Топологические векторные пространства. М.: Мир, 1967.

[59] Н. С. Ландкоф. Основы современной теории потенциала. М.: Наука, 1966.

Институт математики с ВЦ УНЦ РАН,

Поступила в редакцию

Уфа

17.07.2001

E-mail: kra@imat.rb.ru 